RENATO WILBERTO ZILLI

\title{
EFICÁCIA EM LONGO PRAZO DAS GLIFLOZINAS VERSUS GLIPTINAS NO TRATAMENTO DO DIABETES MELLITUS TIPO 2 APÓS FALÊNCIA DA METFORMINA COMO MONOTERAPIA: REVISÃO SISTEMÁTICA E METANÁLISE EM REDE
}

Tese apresentada ao Programa de Ciências Médicas da Faculdade de Medicina da Universidade de São Paulo para obtenção do título de Doutor em Ciências.

Área de Concentração: Processos Imunes e Infecciosos

Orientador: Prof. Dr. Fabiano Pinheiro da Silva

(Versão corrigida. Resolução CoPGr 6018/11, de 13 de outubro de 2011. A versão original está disponível na Biblioteca da FMUSP)

São Paulo 
Dados Internacionais de Catalogação na Publicação (CIP)

Preparada pela Biblioteca da

Faculdade de Medicina da Universidade de São Paulo

Creprodução autorizada pelo autor

Zilli, Renato Wilberto

Eficácia em longo prazo das gliflozinas versus gliptinas no tratamento do diabetes mellitus tipo 2 após falência da metformina como monoterapia : revisão sistemática e metanálise em rede / Renato Wilberto Zilli -- São Paulo, 2017.

Tese(doutorado)--Faculdade de Medicina da Universidade de São Paulo. Programa de Ciências Médicas. Área de concentração: Processos Imunes e Infecciosos.

Orientador: Fabiano Pinheiro da Silva.

Descritores: 1.Diabetes mellitus tipo 2 2.Metanálise 3.Terapia combinada 4.Falha de tratamento 5.Metformina 6.Inibidores da dipeptidil peptidase IV 7.Transportador 2 de glucose-sódio/inibidores 8.Empagliflozina 9.Dapagliflozina 10.Saxagliptina 
Esta tese de doutorado está de acordo com as seguintes normas, em vigor no momento desta publicação:

Referências: adaptado de International Committee of Medical Journals Editors (Vancouver).

Guia de apresentação e dissertações, teses e monografias.

Elaborado por Anneliese Cordeiro da Cunha, Maria Julia de A.L. Freddi, Maria F. Crestana, Marinalva de Souza Aragão, Suely Campos Cardoso, Valéria Vilhena. $3^{a}$ Ed. São Paulo: Divisão de Biblioteca e Documentações; 2012

Abreviaturas dos títulos dos periódicos de acordo com List Journals Indexed in Index Medicus. 
Dedico esta tese a minha mãe, Rosita Machado, uma mulher vitoriosa que eu admiro. 


\section{AGRADECIMENTOS}

Agradeço ao meu orientador, Prof. Dr. Fabiano Pinheiro, pela sua dedicação, por sempre estar disposto a colaborar.

Ao Dr. Renato Baena, pelo seu tempo, conhecimento e fins de semana!

A Rodrigo Brandão, Marcelo Silva, Anna Buehler que me ajudaram nas etapas iniciais da minha tese, obrigado por disporem de seu tempo e conhecimento.

A Cochrane do Brasil, onde tive oportunidade de aprender sobre revisão sistemática.

A Claudio Tavares por me ajudar com os pequenos e importantes detalhes da minha tese.

À minha família, amigos e pacientes! Prometo dedicar mais de mim a vocês agora. 
Zilli RW. Eficácia em longo prazo das gliflozinas versus gliptinas no tratamento do diabetes mellitus tipo 2 após falência da metformina como monoterapia: revisão sistemática e metanálise em rede [tese]. São Paulo: Faculdade de Medicina, Universidade de São Paulo; 2017.

\section{RESUMO}

A metformina é a droga de escolha no tratamento inicial do diabetes mellitus tipo 2 (DM2). Não existe consenso na literatura sobre qual seria a segunda melhor opção terapêutica após a falência desta em longo prazo. Objetivo: avaliar a eficácia em longo prazo de gliflozinas e gliptinas após a falência do tratamento primário com metformina no DM2. Material e métodos: foi realizada uma revisão sistemática para o maior tempo de tratamento nas bases de dados bases Embase, Pubmed (via Medline), Lilacs e Cochrane Library e metanálise em rede com as sulfoniluréias (glimepirida e glipizida) como meta comparador. Desfechos: eficácia da medicação (valor final da HbA1c e porcentagem de pacientes com $\mathrm{HbA} 1 \mathrm{c}<7 \%$ ), variação de peso e frequência de pacientes com hipoglicemia. Resultados: O maior tempo de segmento foi de quatro anos. Foram selecionados um artigo com empagliflozina, um artigo com dapagliflozina e um artigo com saxagliptina com dados faltantes. Após um ano de tratamento, mais de $50 \%$ dos pacientes estavam com $\mathrm{HbA} 1 \mathrm{c}>7 \%$. $\mathrm{O}$ perfil de eficácia em quatro anos da empagliflozina (23\%) foi melhor que da dapagliflozina $(5 \%)$ e saxagliptina $(7 \%)$, porém com valores de HbA1c não estatisticamente significantes (7,4 e 7,3\% entre as gliflozinas), sem dados para a saxagliptina. Entretanto, a empagliflozina foi superior à glimepirida no período de quatro anos (diferença média padronizada/DMP: 0,40, intervalo de confiança/IC95\%: 0,23- 0,56). A variação de peso permaneceu estável após um ano de tratamento, com vantagem em quatro anos para a empa (DMP: 1,56, IC95\%: 1,23-1,88). A frequência de pacientes com hipoglicemia não diferiu entre empagliflozina e dapagliflozina (razão de chances: 1,53, IC95\%: 0,80-2,91) e foi significativamente menor do que em relação às sulfoniluréias. Conclusões: a falência da segunda terapia com gliflozinas ocorre em menos de um ano de tratamento ( $>50 \%$ dos pacientes com $\mathrm{HbA} 1 \mathrm{c}>7 \%$ ). A empagliflozina obteve um controle glicêmico melhor em relação às sulfoniluréias, porém semelhante à dapagliflozina. A perda de peso foi 
mantida por quatro anos, com superioridade para empagliflozina. Houve uma baixa frequência de hipoglicemia nas gliflozinas em comparação com as sulfoniluréias. Mais estudos são necessários para avaliar a eficácia de gliptinas e gliflozinas em longo prazo, após a falência terapêutica com metformina.

Descritores: 1.diabetes mellitus tipo 2; 2.metanálise; 3.terapia combinada; 4.falha de tratamento; 5.metformina; 6.inibidores da dipeptidil peptidase IV;7.transportador 2 de glucose-sódio /inibidores; 8.empagliflozina; 9.dapagliflozina; 10.saxagliptina 
Zilli RW. Long-term efficacy of gliflozins versus gliptins in the treatment of type 2 diabetes mellitus after metformin failure as monotherapy: systematic review and network meta-analysis [thesis]. São Paulo: "Faculdade de Medicina, Universidade de São Paulo"; 2017.

\section{ABSTRACT}

Metformin is the first-choice treatment in people with type 2 diabetes (TD2). There is no consensus in the medical literature about which drug would be a second-best option of treatment in the case of metformin failure in long-term. Objective: to assess the long-term efficacy of gliflozins and gliptins once metformin has failed as the primary treatment for TD2. Materials and methods: a systematic review was performed considering the longest period found in Embase, Pubmed (via Medline), Lilacs and Cochrane Library databases and also network meta-analyses using sulfonylureas (glimepiride and glipizide) as a meta comparator. Clinical outcomes where efficacy of medical treatment (final value of $\mathrm{HbA} 1 \mathrm{c}$ and percentage of patients with $\mathrm{HbA} 1 \mathrm{c}<7 \%$ ), weight variation and frequency of patients with hypoglycemia. Results: the longest period of the segment was 4 years. It was selected 1 article on empagliflozin, 1 article on dapagliflozin, and 1 article on saxagliptin with missing data. After one year of treatment, over $50 \%$ of the patients presented $\mathrm{HbA} 1 \mathrm{c}>7 \%$. Efficacy rate in 4 years of empagliflozin (23\%) was better than dapagliflozin (5\%) and saxagliptin $(7 \%)$, however presenting statistically non-significant values for $\mathrm{HbA} 1 \mathrm{c}$ (7.4 and $7.3 \%$ between gliflozins), and missing data for the saxaglifozin. Nonetheless, empagliflozin performed better than glimepiride in the 4-year period (standardized mean difference SMD 0.4, confidence interval $\mathrm{Cl} 95 \% 0.23$ to 0.56). Weight variation remained stable after one year of treatment, presenting empagliflozin better results in the 4-year period (SMD 1.56, Cl 95\% 1.23 to 1.88). The frequency of patients with hyperglycemia did not vary for empagliflozin and dapagliflozin (odds ratio $1.53, \mathrm{Cl} 95 \% 0.8$ to 2.91 ), and it was significantly lower when compared to the sulfonylureas. Conclusions: the failure of the secondary treatment using gliflozins occurs in less than one year of treatment (less than $50 \%$ of the patients presenting $\mathrm{HbA} 1 \mathrm{c}>7 \%$ ). Empagliflozin offered a better glycemic control compared to the sulfonylureas, but similar to dapagliflozin. The weight loss was 
maintained for 4 years, being empagliflozin the one with better results. There was a low frequency of hypoglycemia for the gliflozins when compared to the sulfonylureas. Further studies are required to evaluate the efficacy of gliptins and gliflozins in the long-term after metformin failure.

Descriptors: 1.diabetes mellitus, type 2; 2.meta-analysis; 3.combined modality therapy; 4.treatment failure; 5.metformin; 6.dipeptidyl-peptidase IV inhibitors; 7.sodium-glucose transporter 2/inhibitors; 8.empagliflozin; 9.dapagliflozin; 10.saxagliptin 


\section{LISTA DE FIGURAS}

Figura 1 - Fluxo de seleção dos artigos incluídos 15

Figura 2 - Representação gráfica do escalonamento da medicação para cada estudo ao longo do tempo 20

\section{LISTA DE GRÁFICOS}

Gráfico 1 - Distribuição temporal dos artigos por classe de droga .......................16

Gráfico 2 - Evolução HbA1c, utilizando valores basais de HbA1c .......................... 25

Gráfico 3 - Variação HbA1c em relação ao basal ........................................... 25

Gráfico 4 - Representação gráfica da metanálise para $\mathrm{HbA1c}$ em quatro anos ...... 28

Gráfico 5 - Evolução da variação de peso nos estudos DAPA e EMPA ................. 30

Gráfico 6 - Representação gráfica da metanálise de variação do peso em relação ao basal em quatro anos ........................................................... 32

Gráfico 7 - Representação gráfica da frequência de pacientes com eventos de hipoglicemia

Gráfico 8 - Representação gráfica da metanálise de frequência de pacientes com hipoglicemia nos estudos DAPA e EMPA 36

\section{LISTA DE TABELAS}

Tabela 1 - Critérios para o diagnóstico de diabetes 01

Tabela 2 - Estudos selecionados a partir da estratégia base de dados ................. 16

Tabela 3 - Características de base por grupo nos estudos avaliados .................... 18 Tabela 4 - Critério inicial de entrada de HbA1c e dose inicial de metformina e titulação

Tabela 5 - Número total de participantes inicias e finais e número de pacientes ao final do estudo que não receberam terapia de resgate 22

Tabela 6 - Evolução temporal da HbA1c (\%) sem terapia de resgate .................... 24

Tabela 7 - Estimativa de pacientes com HbA1c < 7\% ao longo do segmento ........ 26

Tabela 8 - Teste do qui quadreado: proporção de pacientes com $\mathrm{HbA} 1 \mathrm{c}<7 \%$ ao final de quatro anos 
Tabela 9 - Metanálise para $\mathrm{HbA} 1 \mathrm{c}(\%)$ em quatro anos nos estudos DAPA e EMPA 28

Tabela 10 - Variação do peso $(\mathrm{Kg})$ nos estudos ........................................... 30

Tabela 11 Metanálise da variação de peso $(\mathrm{Kg})$ em relação ao basal em quatro anos nos estudos DAPA e EMPA

Tabela 12 - Frequência de pacientes com eventos de hipoglicemia por períodos de tempo

Tabela 13 - Metanálise para hipoglicemia em quatro anos nos estudos DAPA e EMPA 36

Tabela 14 - Risco de viés baseado nas características metodológicas dos estudos incluídos 38

Tabela 15 - Qualidade da evidência 39

\section{LISTA DE ABREVIATURAS, SIGLAS E SÍMBOLOS}

$\begin{array}{ll}\text { ADO } & \text { Antidiabético oral } \\ \text { A } & \text { Alfa } \\ \beta & \text { Beta } \\ \gamma & \text { Gama } \\ \leq & \text { Menor ou igual } \\ \geq & \text { Maior ou igual } \\ \mathbb{B} & \text { Marca registrada } \\ \text { dL } & \text { Decilitros } \\ \text { DM2 } & \text { Diabetes Mellitus tipo 2 } \\ \text { DMP } & \text { Standardized mean difference } \\ \text { DP } & \text { Desvio padrão } \\ \text { GLP-I } & \text { Análogos do peptídeo idêntico ao glicagon I } \\ \text { HbA1C } & \text { Hemoglobina glicada A1c } \\ \text { I }^{2} & \text { Teste de inconsistência Higgins } \\ \text { IC } & \text { Intervalo de confiança } \\ \text { IDF } & \text { International Diabetes Federation } \\ \text { iDPP-4 } & \text { Inibidores da depeptidil pettidase-4 } \\ \text { IMC } & \text { Índice de Massa Corporal }\end{array}$




$\begin{array}{ll}\text { iSGLT2 } & \text { Inibidores seletivos da sódio co-transportador de glicose } 2 \\ \text { Kg } & \text { Kilogramas } \\ \text { LOCF } & \text { Last observation caried forward } \\ \text { M } & \text { Metros } \\ \text { mg } & \text { Miligramas } \\ \text { mg/d } & \text { Miligramas por dia } \\ \text { MMRM } & \text { Mixed effect model repeat measurement } \\ \text { MTC } & \text { Mixed Treatment Comparisons } \\ \text { N } & \text { Tamanho da amostra } \\ \text { OC } & \text { Observed cases } \\ \text { PPAR- } \gamma & \text { Proliferator-activated receptor gama } \\ Q^{2} & \text { Qui-quadrado } \\ \text { RC } & \text { Razão de chances }\end{array}$




\section{SUMÁRIO}

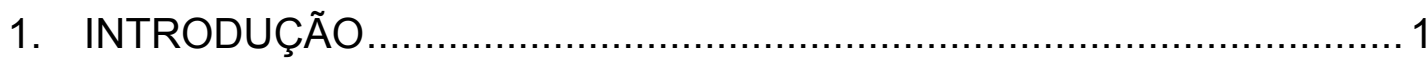

1.1 Escolha da segunda opção na terapia combinada após a falência da

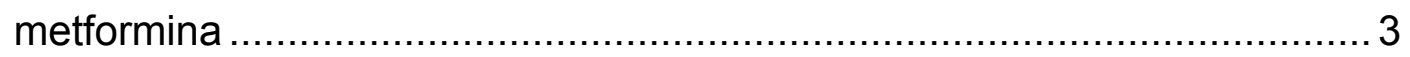

1.2 Durabilidade do controle glicêmico em longo prazo............................... 5

1.3 A revisão sistemática e metanálise em rede como ferramenta de comparação entre classes de medicações ............................................................... 5

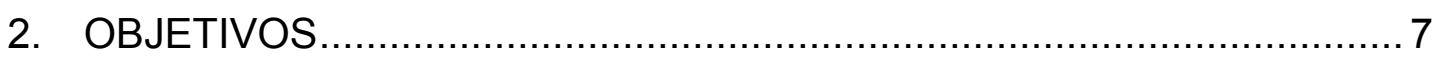

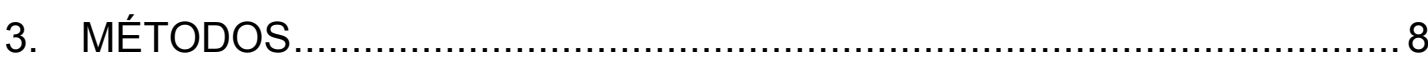

3.1 Definições dos critérios de elegibilidade ....................................... 8

3.2 Bases eletrônicas de dados pesquisadas ......................................... 8

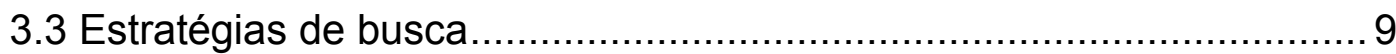

3.4 Avaliação de artigos duplicados na base ........................................ 10

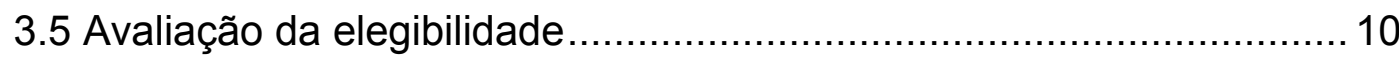

3.6 Avaliação de artigos com textos completos ....................................... 11

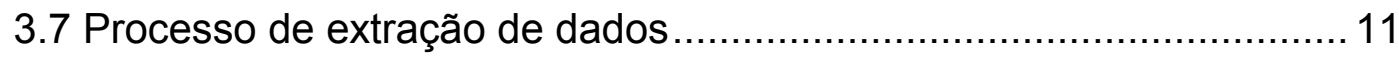

3.8 Plano de análises estatísticas da revisão sistemática e metanálise ... 12

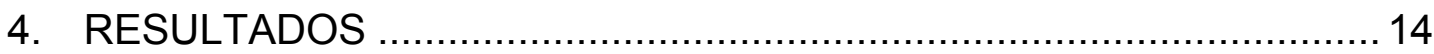

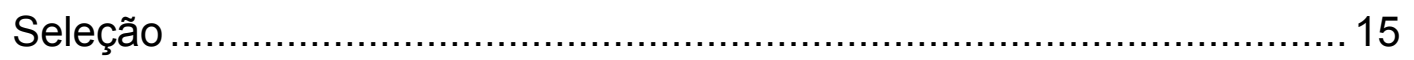

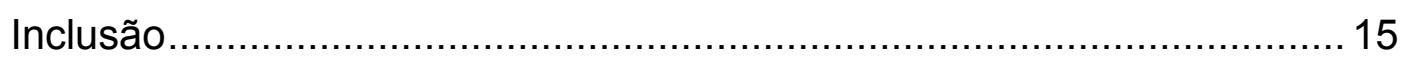

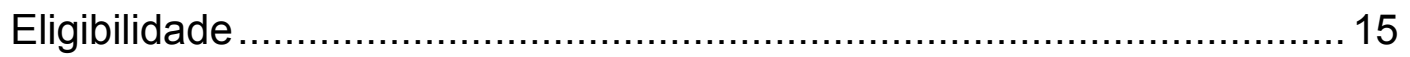

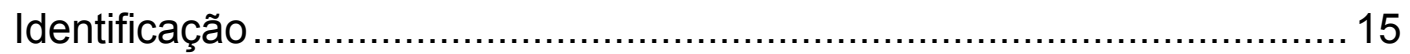

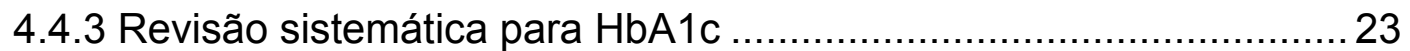

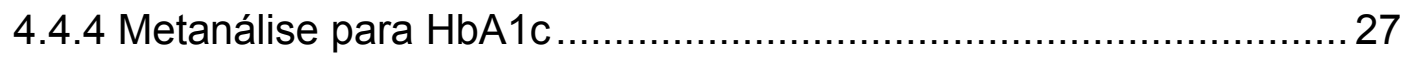

4.4.5 Revisão sistemática em relação à variação do peso ......................... 29

4.4.6 Metanálise em relação à variação do peso ……….......................... 31

4.4.7 Revisão sistemática em relação à frequência de pacientes com hipoglicemia 33

4.4.8 Metanálise da frequência de pacientes com hipoglicemia ............... 35

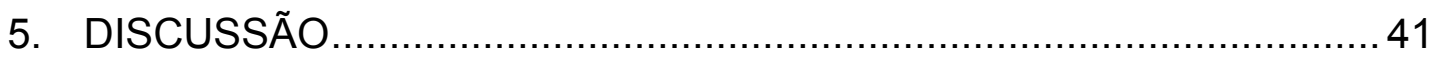

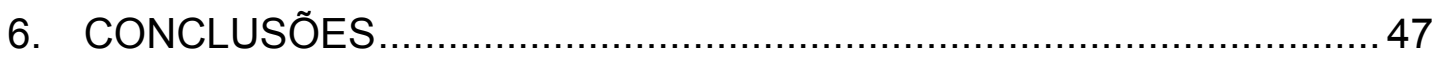

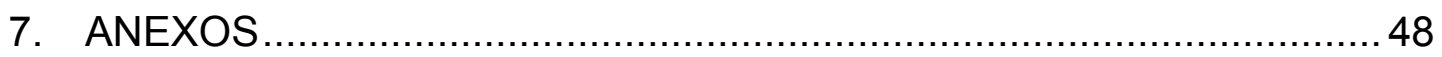

Anexo A. Aprovação Comitê de Ética FMUSP.......................................... 48 
Anexo B. Registro na base PROSPERO .......................................... 49

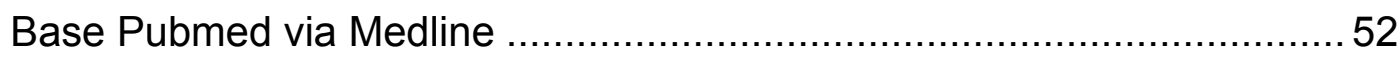

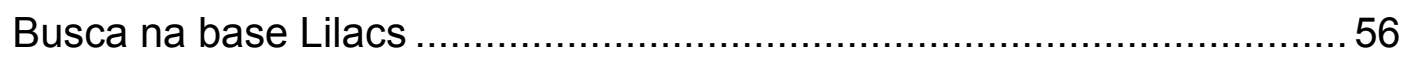

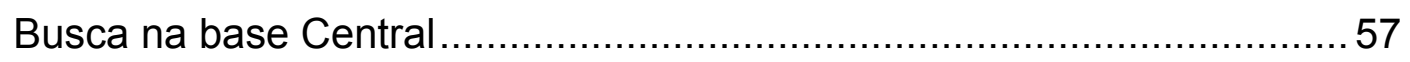

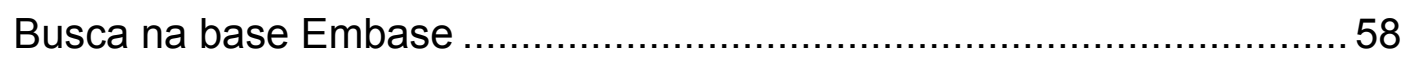

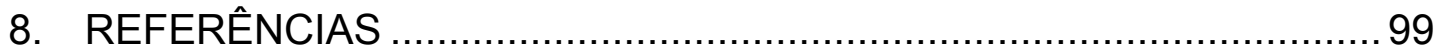





\section{INTRODUÇÃO}

O termo diabetes mellitus engloba um grupo de doenças metabólicas heterogêneas caracterizadas por hiperglicemia como consequência de um defeito na secreção de insulina, sua ação ou ambos (1).

A prevalência global de diabetes em adultos estimada pela Federação Internacional de Diabetes (do inglês - International Diabetes Federation, IDF) em 2013 foi de 8,3\% (382 milhões de pessoas) e esse número deverá subir para além de 592 milhões em 2035, com uma prevalência de 10,1\% da população (2). O Brasil apresenta a quarta maior população de pessoas com diabetes no mundo, segundo a IDF (3). No inquérito nacional de diabetes autorreferido em $2013,6,2 \%$ da população adulta apresentava a doença (4).

As formas de diagnóstico do diabetes mellitus tipo 2 (DM2) podem ser visualizadas na Tabela 1 (5):

\section{Tabela 1 - Critérios para o diagnóstico de diabetes}

- Um valor de glicemia de jejum plasmática $\geq 126 \mathrm{mg} / \mathrm{dL}$. O jejum é definido como nenhuma ingestão calórica durante pelo menos $8 \mathrm{~h}$.

- Um valor de glicemia plasmática $\geq 200 \mathrm{mg} / \mathrm{dL}$, durante um teste oral de tolerância a glicose, utilizando uma carga de glucose contendo o equivalente a $75 \mathrm{~g}$ de glucose anidra dissolvida em água.

- Um valor de $\mathrm{HbA} 1 \mathrm{c} \geq 6,5 \%$.

- Um valor aleatório de glicemia ao acaso $\geq 200 \mathrm{mg} / \mathrm{dL}$ em um paciente com sintomas clássicos de hiperglicemia.

O DM2 corresponde por mais de $90 \%$ dos casos de diabetes, é uma doença tipicamente progressiva, caracterizada pela deterioração gradual da função das células $\beta$ pancreáticas e resistência à insulina de base $(5,6)$. Seu tratamento requer geralmente a terapia escalonada e combinada ao longo do tempo. O foco primário 
da terapia é o controle glicêmico, visando reduzir o risco de complicações micro e macrovasculares $(7,8)$.

A dosagem da hemoglobina glicada $(\mathrm{HbA} 1 \mathrm{c})$ é o principal exame de acompanhamento em longo prazo do controle glicêmico, avalia a glicemia média nos últimos 3 meses, sendo valores menores que 6,5\% o foco primário, podendo ser individualizada para grupos específicos. Cada decréscimo de $1 \%$ no nível da $\mathrm{HbA} 1 \mathrm{c}$ em um paciente descompensado está associado a uma diminuição de aproximadamente $35 \%$ do risco de complicações microvasculares (9-12).

A hipoglicemia, definida como valores de glicemia menores que $70 \mathrm{mg} / \mathrm{dL}$ (13), é um dos fatores limitantes no tratamento, podendo potencializar déficits cognitivos agudos leves, até coma e morte (13). O ganho de peso associado às medicações também é um limitador do tratamento, exigindo ajustes de medicação e dificultando o controle glicêmico em longo prazo (14).

Os consensos para DM2 incluem medidas de mudança do estilo de vida e tratamento medicamentoso para o controle glicêmico inicial, preferencialmente com metformina como terapia isolada (15-18). A metformina é uma droga barata, segura, neutra em relação ao peso, com poucos efeitos colaterais e com um efeito significativo sobre o controle glicêmico $(8,16)$, apesar de na prática clínica não ser a primeira opção em mais de $40 \%$ das vezes (19). Ela reduz a $\mathrm{HbA} 1 \mathrm{c}$ inibindo a produção hepática de glicose $(20,21)$, mas não impede a falência das células $\beta$ pancreáticas $(22,23)$. Em uma coorte observacional, a falência do tratamento com uso inicial de metformina ( $\mathrm{HbA} 1 \mathrm{c}>7,5 \%)$ ocorreu após um período de 27,6 meses (24).

Se as metas de controle glicêmico não são mantidas ao longo do tratamento, a recomendação é a introdução de uma segunda droga, sequencialmente uma terceira droga até a introdução de insulina $(15-17,25,26)$. A inércia clínica, que leva a um retardo na progressão do tratamento de pacientes com diabetes, pode levar a um aumento da prevalência de doenças macrovasculares em longo prazo $(27,28)$. 


\subsection{Escolha da segunda opção na terapia combinada após a falência da metformina}

Atualmente existem no mercado várias classes de medicações orais para o tratamento do diabetes após a falência da metformina, a saber: sulfoniluréias, glitazonas, meglitinidas, inibidores da $\alpha$-glicosidase $e$, mais recentemente, as gliptinas (inibidores da dipeptidil peptidase-4 - iDPP-4) e gliflozinas (inibidores seletivos da sódio-co-transportador de glicose 2 - iSGLT2). Além de medicações injetáveis como as insulinas e os análogos do peptídeo idêntico ao glucagon 1 (GLPI) $(15,16)$.

As drogas com características secretagogas, ou seja, que estimulam a secreção de insulina independente da concentração de glicose podem ser divididas em duas classes, sulfoniluréias e gliptinas. As sulfoniluréias são frequentemente utilizadas após a falência da terapia primária com metformina, porém apresentam risco elevado de hipoglicemia, incluindo $4 \%$ de risco de morte devido a esta $(29,30)$, além de ganho de peso $(31,32)$. Mais recentemente, as glinidas ou meglitinidas foram introduzidas no mercado, em comparação às sulfoniluréias apresentam um período de ação menor e baixo risco de hipoglicemias (33).

As glitazonas ou tiazolinideonas aumentam a sensibilidade à insulina via ativação do PPAR-Y (do inglês - proliferator-activated receptor gama) (34). Apresentam um efeito metabólico mais durador que as sulfoniluréias e a metformina (23). Contudo, seus principais efeitos adversos são ganho de peso, edema e predisposição para insuficiência cardíaca e risco de fraturas ósseas $(23,34)$.

Os análogos do GLP-I são medicações injetáveis, de curta ou longa duração, que mimetizam o efeito do GLP-I endógeno, estimulando a secreção de insulina após a ingestão de glicose e suprimindo o efeito do glucagon pancreático (35). Estão associados à perda de peso e seu principal efeito colateral são náuseas e vômitos (36).

A sitagliptina, primeira droga da classe das gliptinas foi lançada em 2006. Existem atualmente no mercado 6 drogas nesta classe, são elas: sitagliptina, saxagliptina, vildagliptina, linagliptina, alogliptina e anagliptina (esta última somente 
no Japão) (26, 37). Em desenvolvimento as seguintes drogas: teneligliptina, gemigliptina, trelagliptina, gosogliptina, xagliptina, dutogliptina, denagliptina e retagliptina (38-45). Elas aumentam o efeito incretínico in vitro, prolongando a ação do GLP-1 nativo (46). As drogas desta classe apresentam eficácia semelhante na redução de níveis de $\mathrm{HbA} 1 \mathrm{c}$, boa segurança e boa tolerância (46).

Em 2013, as gliflozinas foram introduzidas no mercado. Atualmente, são quatro fármacos disponíveis (canagliflozina, dapagliflozina, ipragliflozina e empagliflozina) $(26,47)$ e seis (remogliflozina, sergliflozina, tofogliflozina, luseogliflozina, bexagliflozina e ertugliflozin) (48-53) em desenvolvimento, estudos clínicos fase II. Estes fármacos atuam aumentando a excreção urinária de glucose de forma independente da secreção de insulina (54) e apresentam eficácia semelhante na redução dos níveis de $\mathrm{HbA} 1 \mathrm{C}$, na segurança e na tolerância. As principais vantagens da classe são: a perda de peso e não causam hipoglicemia quando utilizadas isoladamente (55-58). Uma variação no mecanismo de ação é a sotagliflozina, que inibe o SGLT1 e SGLT2 (59). Em estudos recentes demonstraram benefícios de proteção cardiovascular e morte (60-63).

Por fim, outros agentes menos potentes disponíveis no mercado são: os inibidores da $\alpha$-glicosidade, que retardam a absorção intestinal de glicose (64); o Coles velam, um sequestrador dos ácidos biliares com limitado efeito glicêmico e mecanismo de ação pouco compreendido (65); e a bromocriptina, que também apresenta um modesto efeito na glicemia e mecanismo de ação incerto (66).

Apesar do número de tratamentos disponíveis e suas possíveis combinações, permanece um desafio tanto para médicos quanto para pacientes alcançar um controle glicêmico adequado, bem como, evitar os frequentes efeitos adversos $(7,8$, 15-17). Consequências não intencionais de tratamento tais como hipoglicemia e ganho de peso podem ter um impacto substancial na satisfação, adesão e qualidade de vida dos pacientes $(8,13,14,17,25)$, portanto são considerações importantes na seleção do tratamento. Estudos mostram que a prevalência de obesidade e hipertensão entre pacientes com DM2 é superior à 50\% (67), e, certamente, estes fatores devem ser considerados na escolha terapêutica. Ainda mais importante é a prevalência de hipoglicemia que se aproxima de $40 \%$ em um determinado ano, sendo $7 \%$ com risco de vida $(30,68)$. 


\subsection{Durabilidade do controle glicêmico em longo prazo}

Para dificultar ainda mais a escolha de associação medicamentosa após a falência de metformina, o DM2 é uma doença progressiva (5) que cursa com uma falência medicamentosa inexorável, mas variável para cada classe de droga (23, $69)$.

Estudo coorte retrospectivo com 20.070 pacientes avaliou o tempo de falência da segunda medicação após a falência da monoterapia com metformina e encontrou um tempo médio de falha do tratamento igual a 3,3 anos para as glitazonas, de 2,4 anos para as sulfas e de 1,6 anos para as gliptinas (70).

Drogas com falência terapêutica precoce, antes de completar três anos da associação com a metformina, não parecem constituir a melhor alternativa medicamentosa para o segundo passo da estratégia terapêutica do DM2. O tempo de falência da segunda medicação constitui fator essencial para o sucesso terapêutico desta condição crônica, mas apresenta curso progressivo.

\subsection{A revisão sistemática e metanálise em rede como ferramenta de comparação entre classes de medicações}

Até o momento, algumas revisões sistemáticas e metanálises publicadas comparam a eficácia de diferentes fármacos entre as classes das gliptinas e gliflozinas após a falência do tratamento com metformina (71-77), porém o tempo máximo de análise foi de dois anos (78). Essas duas novas classes terapêuticas recém introduzidas no tratamento para o DM2 precisam de mais estudos, principalmente no que diz respeito ao perfil de segurança e eficácia em longo prazo.

Já existe na literatura médica uma revisão sistemática e metanálise avaliando apenas a segurança das gliptinas e gliflozinas associadas a metformina em estudos com até 24 semanas de duração, porém sem avaliar a eficácia das classes após a falência primária da metformina (79). Por se tratarem de classes novas, a grande maioria dos estudos randomizados utilizou sulfoniluréias como comparador após a falência com a metformina $(55,56,80-91)$ e/ou placebo $(43,53,71,72,75,92-104)$. 
Uma revisão sistemática é um estudo secundário que reúne evidências de estudos primários publicados ou não, e obtidos após pesquisa abrangente na literatura, atendendo a critérios pré-estabelecidos com o objetivo de responder a uma questão específica, estabelecendo o valor dos achados e sintetizando os resultados obtidos (105).

A metanálise é uma metodologia estatística aplicada à revisão sistemática que integra os resultados de dois ou mais estudos primários independentes (105). Ela estima com maior poder e precisão o tamanho do efeito da intervenção, muitas vezes não demonstrado em estudos únicos, ou com metodologia inadequada e tamanho de amostra insuficiente (106).

A eficácia dos tratamentos médicos geralmente é investigada através de comparações diretas em estudos randomizados controlados. Comparações indiretas podem ser necessárias quando duas ou mais opções terapêuticas são comparadas com uma terceira opção em comum. Esta estrutura tem o nome de metanálise MTC (abreviação para a expressão em inglês Mixed Treatment Comparisons) (107), um tipo específico de metanálise em rede muito utilizada na tomada de decisão baseada em evidências científicas (108). 


\section{OBJETIVOS}

Avaliar, por meio de revisão sistemática e metanálise em rede, a eficácia das gliptinas versus gliflozinas com as demais medicações para o tratamento do DM2 após falência do tratamento com metformina.

Desfecho primário:

1) Controle glicêmico (valor de HbA1c e eficácia) em longo prazo (estudos com duração maior que dois anos) em pacientes com DM2 após a falência com metformina e início de terapia combinada com adição de segunda droga. Sendo a eficácia definida pelo percentual de pacientes que completaram o estudo com HbA1c menor ou igual 7\% em relação ao início do tratamento sem adição de uma terceira medicação de resgate.

Desfechos secundários:

1) Variabilidade do peso corporal em longo prazo em pacientes com DM2 após a falência com metformina e início de terapia combinada com a adição da segunda droga.

2) Ocorrência da frequência de pacientes com eventos de hipoglicemia em longo prazo em pacientes com DM2 após a falência com metformina e início de terapia combinada com a adição da segunda droga. 


\section{MÉTODOS}

O estudo foi aprovado pelo Comitê de Ética em Pesquisa da Faculdade de Medicina da Universidade de São Paulo e registrado na base de revisões sistemáticas PROSPERO com o número CRD42015026155 (Anexos A e B respectivamente). A metodologia da revisão sistemática e metanálise foram baseadas nos critérios da Cochrane (105) e apresentada conforme a metodologia Prisma (109).

\subsection{Definições dos critérios de elegibilidade}

$\mathrm{Na}$ avaliação inicial da literatura definiu-se como critérios de elegibilidade pacientes adultos, portadores de diabetes mellitus tipo 2 (diabete melito não insulinodependente). Uso prévio antes da randomização de metformina isoladamente para controle glicêmico em qualquer dose, valores iniciais de $\mathrm{HbA} 1 \mathrm{c}$ maiores que 6,5\% e sendo um dos braços da randomização composto por algum fármaco classificado como alternativa para terapia combinada, segunda droga (gliptina ou gliflozina).

Devido ao diabetes ser uma doença progressiva e com deterioração glicêmica em longo prazo, só foram considerados para análise estudos com duração de 52 semanas ou mais após a randomização, para posterior avaliação do tempo de exposição máxima ao tratamento e tempo de falência.

Estudos com pacientes portadores de diabetes mellitus tipo 1 (diabete melito insulino-dependente), pacientes pediátricos ou menores de 18 anos não foram incluídos.

\subsection{Bases eletrônicas de dados pesquisadas}

A estratégia de busca incluiu as bases: Pubmed via Medline, Embase, Lilacs; e como base secundária de dados, a Cochrane Central Register of Controlled Trials (Central) e o registro na plataforma internacional de registro de ensaios clínicos da 
Organização Mundial de Saúde. As buscas foram realizadas em 20 de julho de 2016, sem limitação de data de procura e idioma.

Para a literatura cinzenta foi realizada busca manual dos anais de 2015 e 2016 do Congresso da Associação Americana de Diabetes, da Sociedade Europeia para Estudos de Diabetes e da Associação Americana do Coração.

\subsection{Estratégias de busca}

A estratégia de busca foi montada conforme a pergunta "PICO", a saber:

- P - população: pacientes com diabetes tipo 2 em uso prévio de metformina isolada para controle glicêmico e falência medicamentosa;

- I - intervenção: em um dos braços da randomização algum fármacos pertencentes à classe das gliptinas ou gliflozinas por pelo menos 52 semanas;

- C - controle: qualquer medicação randomizada, podendo ser medicações orais ou injetáveis, também como placebo; e

- O - desfecho: avaliação da variação da HbA1c, variação de peso e ocorrência de hipoglicemias.

Foram utilizados os descritores de "população" e "intervenção" para a pesquisa bibliográfica, segundo as orientações do Handbook da Cochrane. Buscas com alta sensibilidade e baixa precisão, utilização de texto livre e indexado e utilização de filtros validados para ensaios clínicos randomizados (105).

A estratégia de busca nas bases foi realizada por blocos:

1. Diabetes tipo 2 e termos relacionados;

2. Metformina e termos relacionados;

3. Inclui os unitermos para as bases específicas, sinônimos e nomes dos fármacos pertencentes às classes das gliptinas ou gliflozinas. Foi realizada uma pesquisa prévia na base de dados da Organização Mundial de Saúde para verificação de fármacos ainda em desenvolvimento; 
4. Filtros específicos para cada base, recomendados pela Colaboração Cochrane (105).

As estratégias foram montadas buscando as publicações disponíveis para cada termo individual dentro do bloco e depois somando estes resultados, utilizando o operador booleano de soma OR. Quando finalizada as buscas dos blocos, os mesmos foram combinados de forma a obter a intersecção dos blocos através do operador booleano AND, tornando, assim, a busca mais específica. Finalizando com um filtro para estudo randomizado controlado. As estratégias se encontram no Anexo C.

\subsection{Avaliação de artigos duplicados na base}

Inicialmente, os artigos foram selecionados pela busca estratégica nas bases de dados, analisando-se títulos e resumos através do programa EndNote $X 7$ como ferramenta de cadastro. Excluíram-se os artigos que estavam em duplicata na base de forma automática e de forma manual. Foi necessária, pelo menos, a leitura de todos os títulos e autores dos artigos, para que possíveis publicações repetidas fossem eliminadas da base, já que muitas vezes são publicadas em nome de diferentes autores ou em diferentes revistas.

\subsection{Avaliação da elegibilidade}

A triagem dos artigos para inclusão ou exclusão, baseada nos critérios de elegibilidade pré-definidos, a saber:

1- Ensaio clínico randomizado ou quase randomizado;

2- Pacientes portadores de diabetes mellitus tipo 2 em falência primária do tratamento com metformina;

3- Algum dos braços da randomização com fármacos da classe das gliptinas ou gliflozinas; e

4- Período superior a 52 meses após a randomização. 
Esta etapa foi realizada por dois revisores independentes. Cada dupla avaliou título e resumo (quando disponíveis). Quando um artigo não apresentava resumo, mas o título era sugestivo de elegibilidade, este foi encaminhado para a próxima etapa de obtenção de texto completo. Na etapa de triagem, cada revisor da dupla interpretou o título e resumo e os resultados foram comparados. Se ao menos um revisor considerou o artigo elegível, ou houvesse dúvida sobre a elegibildade, este foi levado para uma reunião de consenso.

\subsection{Avaliação de artigos com textos completos}

Todos os artigos elegíveis pela leitura de título e resumo tiveram sua elegibilidade confirmada por meio da coleta dos critérios de elegibilidade em uma ficha clínica padronizada (Anexo D). A ficha clínica utilizada na avaliação da elegibilidade avaliou os quatro critérios estabelecidos para inclusão dos estudos na revisão do item anterior.

Nestes artigos em texto completo também foram avaliadas outras questões não abordadas na ficha clínica, mas importantes para classificação na revisão, como os desfechos, por exemplo. Não houve restrição de língua na busca estratégica. Nesta etapa foi realizada a avaliação do texto completo, havendo divergências, estas foram solucionadas por consenso.

\subsection{Processo de extração de dados}

A extração foi guiada por ficha padrão (Anexo E). Os artigos elegíveis forneceram os dados para as análises dos desfechos de interesse da revisão. Nos casos de dados não disponíveis os autores foram contatos (Apêndice $A$ ).

Foram avaliadas nos estudos as características de base das populações, variações da $\mathrm{HbA} 1 \mathrm{c}$, peso e valores de hipoglicemia por paciente em cada grupo nos tempos: seis meses, um, dois, três e quatro anos, com maior ênfase no período maior que dois anos. 
Foi planejada a análise de subgrupo em relação a sexo, idade (menor de 65 ou maior/igual a 65 anos) e tempo de diagnóstico do diabetes (menor que três anos ou maior/igual a três anos).

Foi utilizado o programa Digitizelt ${ }^{\circledR}$ para estimativa dos dados não descritos, porém apresentados de forma gráfica: curva de evolução HbA1c e peso no tempo para os estudos envolvendo dapagliflozina (110) e empaglifozina $(91,111)$.

\subsection{Plano de análises estatísticas da revisão sistemática e metanálise}

A análise estatística foi realizada em duas etapas. Na primeira etapa se procedeu à revisão sistemática dos estudos, analisando-se as características de base dos pacientes, definições de desfecho, intervenções e comparadores utilizados.

Como tamanho de efeito (treatment effect or effect size) foram utilizados diferença média padronizada (DMP - standardized mean difference) para as variáveis contínuas ( $\mathrm{HbA1c}$ e variação de peso) e razão de chances ( $\mathrm{RC}$ - Odds Ratio) para as variáveis categóricas (frequência de pacientes com hipoglicemia).

Os resultados foram exibidos em termos de IC 95\% (intervalos de confiança de $95 \%$ ) para cada uma das comparações.

Para o desfecho primário, foi estimada a eficácia terapêutica (percentual de pacientes com $\mathrm{HbA} 1 \mathrm{c} \leq 7 \%$ ao final do estudo) da distribuição normal através da média amostral e desvio padrão na ausência do dado, calculado o porcentual em relação ao início do estudo e realizado o teste qui-quadrado e metanálise dos valores de $\mathrm{HbA} 1 \mathrm{c}$.

Para os desfechos secundários, variação de peso e frequência de pacientes com hipoglicemia, foram realizadas a revisão sistemática e metanálise Bayesiana em rede (112).

Para a confecção dos gráficos foi utilizado o programa Graphpad Prism ${ }^{\circledR} \mathrm{e}$ linguagem R. A metanálise em rede foi realizada utilizando como meta comparador a 
classe das sulfoniluréias. Como ferramenta para o cálculo da metanálise, utilizou-se a linguagem $\mathrm{R}$.

Foi planejado medir a consistência entre os resultados de cada estudo, utilizando-se do teste de inconsistência $\mathrm{I}^{2}$ de Higgins (113). O relatório estatístico completo encontra-se no Apêndice B.

Para avaliação da qualidade da evidência foi utilizada uma variação da tabela de resumo de evidências (summary of findings) proposta pela Cochrane, adaptada para uma metanálise em rede (114). 


\section{RESULTADOS}

\subsection{Resultado da estratégia de busca}

Foram recuperados da base de dados Central 262, Lilacs 2, Embase 1.359, Medline via Pubmed 876, Congresso da Sociedade Europeia para Estudo do Diabetes (EASD), seis referências, e do Reunião científica da Associação Americana de Diabetes (ADA), uma referência. A soma de todas as referências das bases eletrônicas pesquisadas totalizou 2.496 artigos. Destes, foram excluídos 257 artigos em duplicatas, totalizando 2.242 artigos para avaliação da elegibilidade.

\subsection{Avaliação da elegibilidade}

Para avaliação da elegibilidade, contou-se com a participação de cinco duplas de revisores [(RZ)-(RB); (RZ)-(RBN); (RZ-AB); e (RZ-MS)]. Cada dupla avaliou em média 560 artigos utilizando-se da ferramenta online covidence.com, sendo escolhidos 379 para a próxima etapa.

A Figura 1 apresenta o fluxo de obtenção dos artigos que foram considerados elegíveis para a revisão sistemática, segundo a metodologia Prisma. 


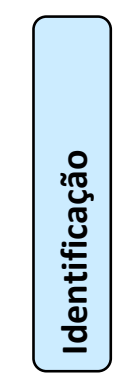
Referencias identificadas através da busca nas bases eletrônicas $(n=2.489)$

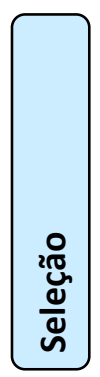

Referências após remoção das duplicatas

$(n=2.242)$

Referências identificadas por busca manual em outras fontes $(n=7)$

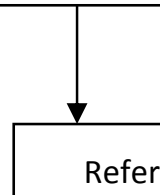

\section{$(n=2.242)$}

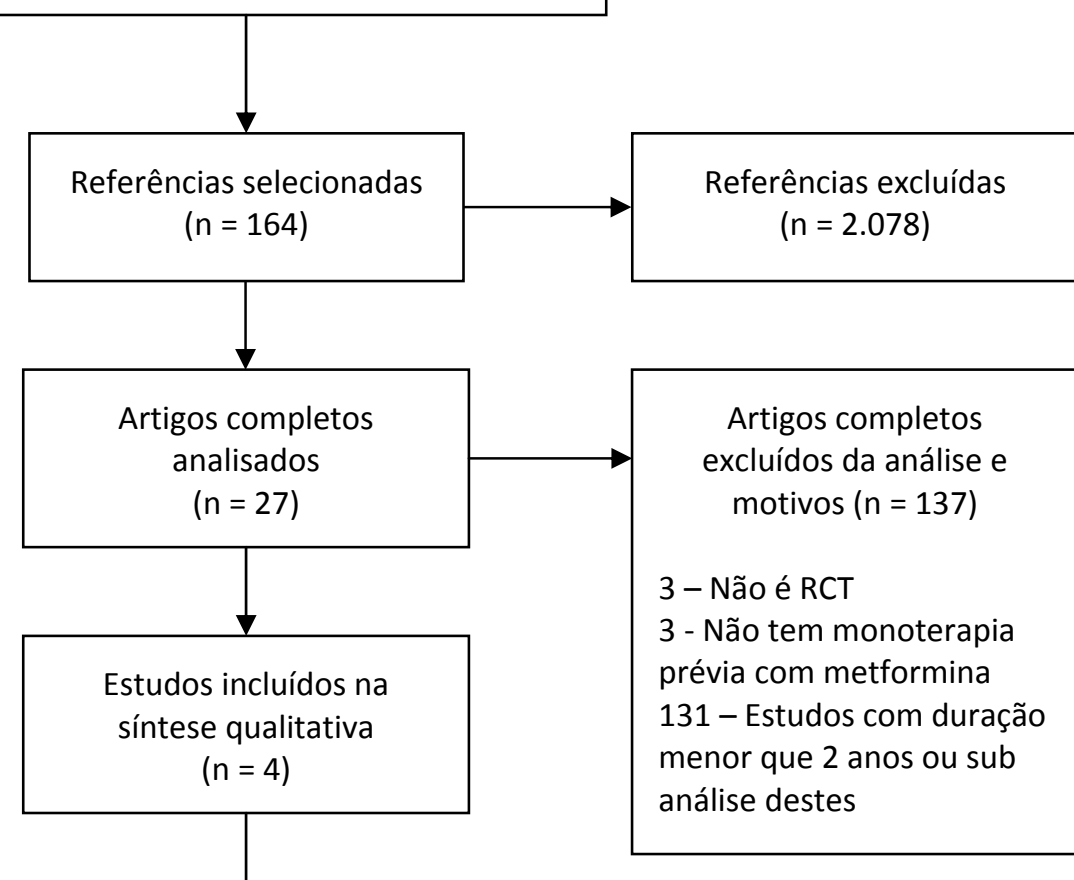

Estudos incluídos na síntese quantitativa (Metanálise) $(n=3)$

Figura 1 - Fluxo de seleção dos artigos incluídos

Fonte: resultado da pesquisa

A razão da exclusão dos artigos completos e os motivos encontram-se no Anexo F. O Gráfico 1 demonstra a relação entre o tempo de randomização, usando como comparador uma droga da classe das gliptinas ou gliflozinas e o número de artigos encontrados. 
Gráfico 1 - Distribuição temporal dos artigos por classe de droga

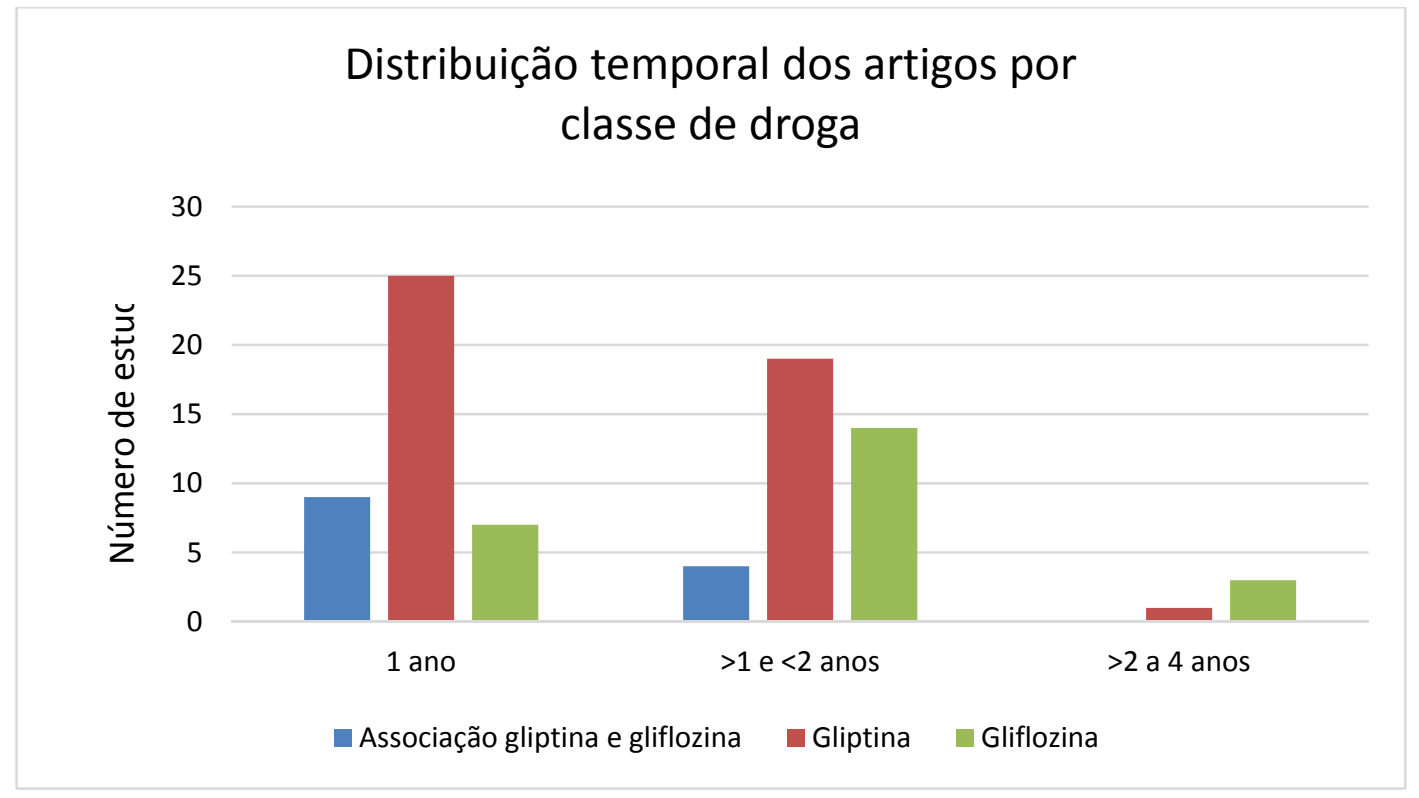

Fonte: resultado da pesquisa

\subsection{Avaliação da extração de dados}

Foram selecionados para a revisão sistemática quatro estudos que atenderam aos critérios pré-selecionados para dapagliflozina (110, 115-121), empagliflozina ( 91 , 111), saxagliptina $(122,123)$ e sitagliptina (124-126), apresentados na Tabela 2. Para facilitar a leitura, os estudos serão abreviados DAPA, EMPA, SAXA e SITA respectivamente. Um resumo das características basais dos estudos, intervenção e desfecho dos estudos selecionados encontram-se no Anexo G.

Tabela 2 - Estudos selecionados a partir da estratégia base de dados

\begin{tabular}{cc|c|c|c}
\hline $\begin{array}{c}\text { Abreviação } \\
\text { Medicação }\end{array}$ & Comparador & $\begin{array}{c}\text { Duração } \\
\text { estudo }\end{array}$ & $\begin{array}{c}\text { Registro } \\
\text { ClinicalTriais }\end{array}$ & $\begin{array}{c}\text { Artigos } \\
\text { selecionados }\end{array}$ \\
\hline DAPA - Dapaglifozina & Glipizida & 4 anos & NCT00660907 & 8 \\
EMPA - Empaglifozina & Glimepirida & 4 anos & NCT01167881 & 2 \\
SAXA - Saxagliptina & Placebo & 4 anos & NCT00121667 & 2 \\
SITA - Sitagliptina & Placebo & 3 anos & NCT00838903 & 3 \\
& Glimepirida & & & \\
& Albuglutida & & & \\
\hline
\end{tabular}

Fonte: resultado da pesquisa 


\subsection{Resultados da revisão sistemática}

\subsubsection{Característica de base dos pacientes nos estudos}

As características da população de interesse em cada estudo são apresentadas na Tabela 3. Todos os estudos foram duplos cegos, randomizados, com alocação em paralelo, com o objetivo primário tratamento.

O estudo DAPA foi concebido para durar dois anos e no seu seguimento foi feita uma extensão opcional de dois anos. O estudo EMPA foi planejado para quatro anos, sendo dois anos de seguimento e mais dois anos opcionais por centro de pesquisa.

Todos os estudos foram financiados pela indústria farmacêutica, seguindo as regras de boas práticas clínicas do Food and Drug Administration dos Estados Unidos. As características referentes à idade, IMC e HbA1c iniciais não foram estatisticamente diferentes (Apêndice B). 
Tabela 3 - Características de base por grupo nos estudos avaliados

\begin{tabular}{|c|c|c|c|c|c|c|c|c|c|c|}
\hline \multirow{4}{*}{$\begin{array}{c}\text { ESTUDO } \\
\text { Tempo } \\
\text { máximo de } \\
\text { intervenção }\end{array}$} & \multicolumn{2}{|c|}{ DAPA } & \multicolumn{2}{|c|}{ EMPA } & \multicolumn{2}{|l|}{ SAXA } & \multicolumn{4}{|c|}{ SITA } \\
\hline & \multicolumn{2}{|c|}{4 anos } & \multicolumn{2}{|c|}{4 anos } & \multicolumn{2}{|c|}{4 anos } & \multicolumn{4}{|c|}{3 anos } \\
\hline & Intervenção & Comparador & Intervenção & Comparador & Intervenção & Comparador & Intervenção & Intervenção & Comparador & Comparador \\
\hline & Dapaflozina & Glipizida & Emplagliflozina & Glimepirida & Saxagliptina & Placebo & Sitagliptina & Albiglutida & Glimepirida & Placebo \\
\hline Pacientes & 400 & 401 & 764 & 775 & 191 & 179 & 302 & 302 & 307 & 101 \\
\hline $\begin{array}{l}\text { HbA1c basal } \\
(\% \pm D P)\end{array}$ & $7,65+0,9$ & $7,74+0,9$ & $7,92+0,8$ & $7,92+0,9$ & $8,07+0,1$ & $8,06+0,1$ & $8,05 \pm 0,8$ & $8,09 \pm 0,8$ & $8,12 \pm 0,8$ & $8,15 \pm 0,9$ \\
\hline $\begin{array}{l}\text { Idade (anos } \\
\pm \text { DP) }\end{array}$ & $58 \pm 9$ & $59 \pm 10$ & $56,2 \pm 10$ & $55,7 \pm 10$ & $54,7 \pm 10$ & $54,8 \pm 10$ & $54,3 \pm 10$ & $54,3 \pm 10$ & $54,4 \pm 10$ & $56,1 \pm 10$ \\
\hline Homens - n & 221 & 220 & 432 & 421 & 103 & 96 & 139 & 135 & 158 & 50 \\
\hline Brancos - $n$ & 327 & 323 & 498 & 519 & 159 & 150 & 226 & 217 & 229 & 65 \\
\hline Negros - $\mathrm{n}$ & 26 & 24 & 12 & 8 & 11 & 7 & 35 & 53 & 39 & 23 \\
\hline Asiáticos - $\mathrm{n}$ & 27 & 34 & 254 & 253 & 3 & 4 & 20 & 18 & 16 & 5 \\
\hline Outros - n & 20 & 20 & 1 & 0 & 18 & 18 & 22 & 17 & 25 & 10 \\
\hline $\begin{array}{l}\text { Duração do } \\
\text { diabetes } \\
\text { (anos } \pm \text { DP) }\end{array}$ & $6 \pm 5$ & $7 \pm 6$ & - & - & & & $6,7 \pm 7$ & $6,0 \pm 4$ & $6,0 \pm 5$ & $6,7 \pm 7$ \\
\hline $\begin{array}{l}\text { Peso inicial } \\
(\mathrm{Kg} \pm \mathrm{DP})\end{array}$ & - & - & $82,5+19$ & $83,0+19$ & $87,26 \pm 17$ & $87,1 \pm 17$ & $91,6 \pm 19$ & $89,6 \pm 18$ & $91,8 \pm 20$ & $91,6 \pm 19$ \\
\hline $\begin{array}{l}\mathrm{IMC}\left(\mathrm{Kg} / \mathrm{m}^{2}\right. \\
\pm \mathrm{DP})\end{array}$ & $31,7 \pm 5$ & $31,2 \pm 5$ & $30,0+5$ & $30,3 \pm 5$ & $31,23 \pm 5$ & $31,6 \pm 5$ & $32,8 \pm 5$ & $32,7 \pm 6$ & $32,5 \pm 5$ & $32,8 \pm 5$ \\
\hline $\begin{array}{l}\text { Hipertensão } \\
\text { - n (\%) }\end{array}$ & $282(70)$ & $282(70)$ & $469(61)$ & $468(60)$ & - & - & $172(57)$ & $193(64)$ & $185(60)$ & $65(64)$ \\
\hline $\begin{array}{l}\text { Dislipidemia } \\
-\mathrm{n}(\%)\end{array}$ & $245(61)$ & $239(60)$ & $411(54)$ & $397(51)$ & - & - & - & - & - & - \\
\hline
\end{tabular}

Resultados apresentados em média caso não especificado. DP: desvio padrão, n: número amostral

O peso inicial do estudo DAPA não foi disponibilizado pelos autores, o tempo de diagnóstico no EMPA foi categorizado em bandas. Não há referência a dados de hipertensão

e dislipidemia no estudo SAXA e dislipidemia no estudo SITA.

Fonte: resultado da pesquisa 
A faixa de inclusão de $\mathrm{HbA} 1 \mathrm{c}$ basal e a definição de falência da terapia com metformina são apresentadas na Tabela 4.

Tabela 4 - Critério inicial de entrada de HbA1c e dose inicial de metformina e titulação

\begin{tabular}{|c|c|c|}
\hline Estudo & $\begin{array}{l}\text { Definição de } \\
\text { falência do } \\
\text { tratamento com } \\
\text { metformina } \\
\text { (HbA1c) }\end{array}$ & Dose inicial de metformina e titulação \\
\hline DAPA & $>6,5 \%$ e $\leq 10 \%$ & $\begin{array}{l}\text { Período de titulação aberta de } 8 \text { semanas. Se o } \\
\text { paciente fazia uso prévio somente de metformina } \\
\text { em dose }<1.500 \mathrm{mg} / \mathrm{d} \text {, aumento progressivo até a } \\
\text { dose de } 1.500 \mathrm{mg} / \mathrm{d} \text {. Se dose maior, manutenção } \\
\text { da dose. Se em uso prévio de outro ADO, retirada } \\
\text { deste e mesmo critério para ajuste dose } \\
\text { metformina que grupo metformina isolada. }\end{array}$ \\
\hline EMPA & $\geq 7 \%$ e $\leq 10 \%$ & $\begin{array}{l}\text { Metformina } \geq 1500 \mathrm{mg} / \mathrm{d} \text { ou a máxima dose } \\
\text { tolerada, ou máxima dose conforme bula local com } \\
\text { dose estável pelo menos } 12 \text { semanas da } \\
\text { randomização }\end{array}$ \\
\hline SAXA & $>7 \%$ e $<10 \%$ & $\begin{array}{l}\text { Dose estável de metformina }(>1,500 \text { e }<2,550 \\
\mathrm{mg} / \mathrm{d}) \text { por pelo menos } 8 \text { semanas da triagem }\end{array}$ \\
\hline SITA & $>7 \%$ e $<10 \%$ & $\begin{array}{l}\text { Dose estável de metformina }(>1,500 \mathrm{mg} \text { ou a } \\
\text { máxima dose tolerada) por }>3 \text { meses antes da } \\
\text { triagem }\end{array}$ \\
\hline
\end{tabular}

ADO - antidiabético oral

Fonte: resultado da pesquisa

Os estudos EMPA, DAPA e SAXA selecionados indicam que houve uma orientação inicial em relação à dieta para diabetes tipo 2 e atividade física, sem outras considerações. O estudo SITA fez recomendações de dieta e atividade físca (conforme recomendações da Associação Americana de Diabetes) que foram realizadas em todas as visitas, sendo responsabilidade dos centros de pesquisa locais definir as políticas utilizadas. 
Cada estudo teve sua particularidade quanto ao aumento de dose da intervenção e do comparador, também como a definição de falência com necessidade de terapia adicional. A Figura 2 ilustra a progressão da dose da medicação durante os estudos. No Anexo H são especificadas a titulação das drogas e definição de falência e resgate terapêutico.

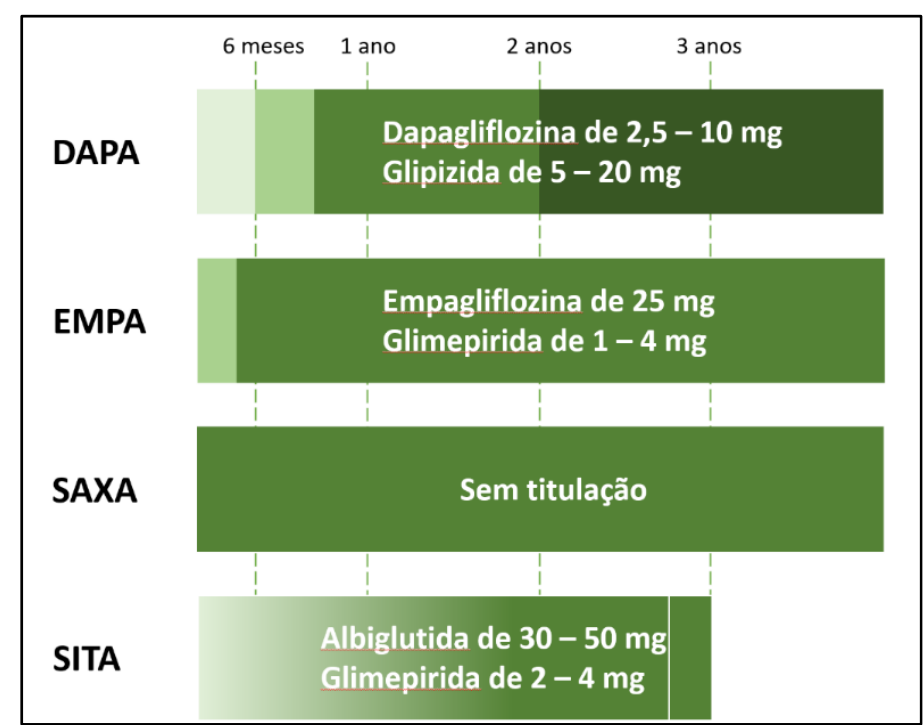

Figura 2 - Representação gráfica do escalonamento da medicação para cada estudo ao longo do tempo

Quanto maior a intensidade da cor, maior a chance de ajuste da medicação, conforme critério de cada estudo. No interior das barras encontram-se a dose mínima e máxima possíveis de titulação.

Fonte: resultado da pesquisa

No estudo DAPA, em dois anos a dose média de dapagliflozina foi de 9,2 $\mathrm{mg}$ e glipizida 15,9 mg (116). Em quatro anos, 79\% dos pacientes alcançaram a dose máxima de dapagliflozina de $10 \mathrm{mg}$ e $63 \%$ dos pacientes no braço glipizida receberam a dose máxima do estudo de $20 \mathrm{mg}$ (110).

Não existem dados de quatro anos referentes ao braço glimepirida do estudo EMPA, porém na publicação de dois anos, somente $40 \%$ dos pacientes do grupo glimepirida utilizaram a dose máxima permitida no estudo de $4 \mathrm{mg}$, enquanto as 
doses de 1, 2 e $3 \mathrm{mg}$ foram utilizadas por 26,16 e $17 \%$ da população respectivamente $(91)$.

\subsubsection{Critérios de exclusão, hipoglicemia e perda de segmento}

Cada estudo classificou de forma diferente os pacientes que compuseram o perfil de segurança, também como os critérios de exclusão (efeito adverso grave, quebra no termo de consentimento e perda de segmento), nem sempre descrito nas publicações. Os critérios de hipoglicemias também variavam entre os estudos e são apresentados no Anexo I.

O número inicial e final de participantes, bem como o número final de pacientes que não necessitaram resgate ao final dos estudos estão demonstrados na Tabela 5. O número final de participantes por braço terapêutico variou entre a 54 a $81 \%$ da amostra inicial, enquanto que o número final de pacientes por braço sem resgate variou de 0 a $48 \%$. 
Tabela 5 - Número total de participantes inicias e finais e número de pacientes ao final do estudo que não receberam terapia de resgate

\begin{tabular}{|c|c|c|c|c|c|c|c|c|c|c|c|c|c|c|c|c|c|c|c|c|c|c|c|c|c|c|c|c|c|c|}
\hline \multirow{4}{*}{ ESTUDO } & \multicolumn{6}{|c|}{ DAPA } & \multicolumn{6}{|c|}{ EMPA } & \multicolumn{6}{|c|}{ SAXA } & \multicolumn{12}{|c|}{ SITA } \\
\hline & \multicolumn{3}{|c|}{ Intervenção } & \multicolumn{3}{|c|}{ Comparador } & \multicolumn{3}{|c|}{ Intervenção } & \multicolumn{3}{|c|}{ Comparador } & \multicolumn{3}{|c|}{ Intervenção } & \multicolumn{3}{|c|}{ Comparador } & \multicolumn{3}{|c|}{ Intervenção } & \multicolumn{3}{|c|}{ Intervençãor } & \multicolumn{3}{|c|}{ Comparador } & \multicolumn{3}{|c|}{ Comparador } \\
\hline & & pafloz & & & lipizid & & Em & gliflo & & & mepi & & & xaglip & & & Place & & & piltip & & & bigluti & & & mepiri & & & laceb & \\
\hline & 1 & $\mathrm{~F}$ & $\%$ & 1 & $\mathrm{~F}$ & $\%$ & 1 & $\mathrm{~F}$ & $\%$ & 1 & $\mathrm{~F}$ & $\%$ & 1 & $\mathrm{~F}$ & $\%$ & $\mathrm{I}$ & $\mathrm{F}$ & $\%$ & 1 & $\mathrm{~F}$ & $\%$ & 1 & $\mathrm{~F}$ & $\%$ & 1 & $\mathrm{~F}$ & $\%$ & 1 & $\mathrm{~F}$ & $\%$ \\
\hline $\begin{array}{l}\text { Amostra de } \\
\text { segurança }\end{array}$ & 400 & 322 & 81 & 401 & 314 & 78 & 765 & 610 & 80 & 780 & 589 & 76 & 191 & 143 & 75 & 179 & 112 & 63 & 302 & 190 & 62,9 & 302 & 192 & 64 & 307 & 191 & 62,2 & 101 & 55 & 54 \\
\hline $\begin{array}{l}\text { Amostra } \\
\text { sem } \\
\text { resgate }\end{array}$ & 400 & 79 & 20 & 401 & 71 & 18 & 765 & 365 & 48 & 780 & 243 & 31 & 191 & 18 & 9 & 179 & 7 & 4 & 302 & 2 & 0,7 & 302 & 0 & 0 & 307 & 2 & 0,7 & 101 & 0 & 0 \\
\hline
\end{tabular}

$\mathrm{I}=$ inicial; $\mathrm{F}=$ final

Fonte: resultado da pesquisa 


\subsubsection{Revisão sistemática para HbA1c}

A evolução do controle glicêmico sem resgate foi avaliada periodicamente em cada estudo, foram coletados dados referentes aos tempos basal, seis meses (24-26 semanas), um, dois, três e quatro anos apresentados na Tabela 6. Apesar do estudo SITA ter tido duração de três anos, na metodologia OC (observed cases), todos os pacientes foram resgatados antes do término deste e por esta razão este estudo foi excluído das análises subsequentes. 
Tabela 6 - Evolução temporal da HbA1c (\%) sem terapia de resgate

\begin{tabular}{|c|c|c|c|c|c|c|c|c|c|c|c|c|c|c|c|c|c|c|c|c|c|c|c|c|c|c|c|c|c|c|}
\hline \multirow{3}{*}{$\begin{array}{l}\text { ESTUDO } \\
\text { Tempo (em } \\
\text { anos) }\end{array}$} & \multicolumn{6}{|c|}{ DAPA } & \multicolumn{6}{|c|}{ EMPA } & \multicolumn{6}{|c|}{ SAXA } & \multicolumn{12}{|c|}{ SITA } \\
\hline & \multicolumn{3}{|c|}{$\begin{array}{l}\text { Intervenção } \\
\text { Dapaglifozina }\end{array}$} & \multicolumn{3}{|c|}{$\begin{array}{l}\text { Comparador } \\
\text { Glipizida }\end{array}$} & \multicolumn{3}{|c|}{$\begin{array}{l}\text { Intervenção } \\
\text { Empaglifozina }\end{array}$} & \multicolumn{3}{|c|}{$\begin{array}{l}\text { Comparador } \\
\text { Glimepirida }\end{array}$} & \multicolumn{3}{|c|}{$\begin{array}{l}\text { Intervenção } \\
\text { Saxagliptina }\end{array}$} & \multicolumn{3}{|c|}{$\begin{array}{c}\text { Comparador } \\
\text { Placebo }\end{array}$} & \multicolumn{3}{|c|}{$\begin{array}{l}\text { Intervenção } \\
\text { Sitagliptina }\end{array}$} & \multicolumn{3}{|c|}{$\begin{array}{l}\text { Intervenção } \\
\text { Albiglutida }\end{array}$} & \multicolumn{3}{|c|}{$\begin{array}{l}\text { Comparador } \\
\text { Glimepirida }\end{array}$} & \multicolumn{3}{|c|}{$\begin{array}{c}\text { Comparador } \\
\text { Placebo }\end{array}$} \\
\hline & Valor & DP & $\mathrm{n}$ & Valor & DP & $\mathrm{n}$ & Valor & $\mathrm{DP}$ & $\mathrm{n}$ & Valor & DP & $\mathrm{n}$ & Valor & DP & $\mathrm{n}$ & Valor & DP & $\mathrm{n}$ & Valor & DP & $\mathrm{n}$ & Valor & DP & $\mathrm{n}$ & Valor & DP & $\mathrm{n}$ & Valor & DP & \\
\hline 0 & 7,65 & 0,90 & 400 & 7,74 & 0,90 & 401 & 7,92 & 0,81 & 765 & 7,92 & 0,86 & 780 & 8,07 & 0,06 & 186 & 8,06 & 0,07 & 175 & 8,05 & 0,8 & 302 & 8,09 & 0,8 & 302 & 8,12 & 0,84 & 307 & 8,15 & 0,9 & 10 \\
\hline 0,5 & 7,16 & 0,64 & 354 & 6,92 & 0,63 & 354 & 6,81 & 0,81 & 702 & 6,73 & 0,81 & 699 & 6,96 & 0,07 & 186 & 7,78 & 0,07 & 175 & $-0,6$ & 0,98 & 267 & $-0,9$ & 0,79 & 265 & $-0,9$ & 0,87 & 271 & $-0,2$ & 0,81 & \\
\hline 1 & 7,13 & 0,73 & 321 & 7,13 & 0,72 & 315 & 6,92 & 0,65 & 645 & 6,99 & 0,63 & 609 & - & - & - & - & - & - & $-0,7$ & 1,16 & 244 & $-0,9$ & 0.95 & 244 & $-0,8$ & 0,96 & 246 & $-0,3$ & 0,93 & \\
\hline 2 & 7,33 & 0,86 & 233 & 7,51 & 0,81 & 208 & 6,99 & 0,71 & 545 & 7,10 & 0,65 & 457 & - & - & - & - & - & - & $-0,6$ & 1,17 & 207 & $-0,9$ & 0,96 & 214 & $-0,8$ & 1,17 & 216 & $-0,4$ & 1,11 & \\
\hline 3 & 7,52 & 0,64 & 105 & 7,72 & 0,64 & 102 & 7,05 & 0,41 & 413 & 7,21 & 0,58 & 365 & $-0,4$ & - & - & 0,1 & - & - & - & - & - & - & - & - & - & - & - & - & - & \\
\hline 4 & 7,45 & 0,68 & 79 & 7,55 & 0,64 & 71 & 7,27 & 0,39 & 365 & 7,44 & 0,48 & 243 & - & - & - & - & - & - & & & & & & & & & & & & \\
\hline
\end{tabular}

Valores de médios de HbA1c e desvio padrão (DP) estimados através de digitalização de gráficos nos períodos de seis meses, três anos do estudo DAPA e EMPA; e quatro anos do estudo EMPA.

O estudo DAPA utilizou a metodologia LOCF (last observation caried forward), estudo EMPA utilizou MMRM (mixed effect model repeat measurement) e estudo SITA utilizou OC (observed cases) para os dados incompletos durante o segmento.

Cada estudo utilizou valores de corte superior para HbA1c que variaram ao longo do estudo.

Fonte: resultado da pesquisa 
Os Gráficos 2 e 3 demostram a evolução da HbA1c no tempo no estudo DAPA e EMPA. Os dados foram extraídos das publicações e dos valores obtidos a partir das digitalizações dos gráficos. O estudo SAXA não disponibilizou os dados evolutivos da HbA1c.

Gráfico 2 - Evolução HbA1c, utilizando valores basais de HbA1c

\section{Evolução HbA1c}

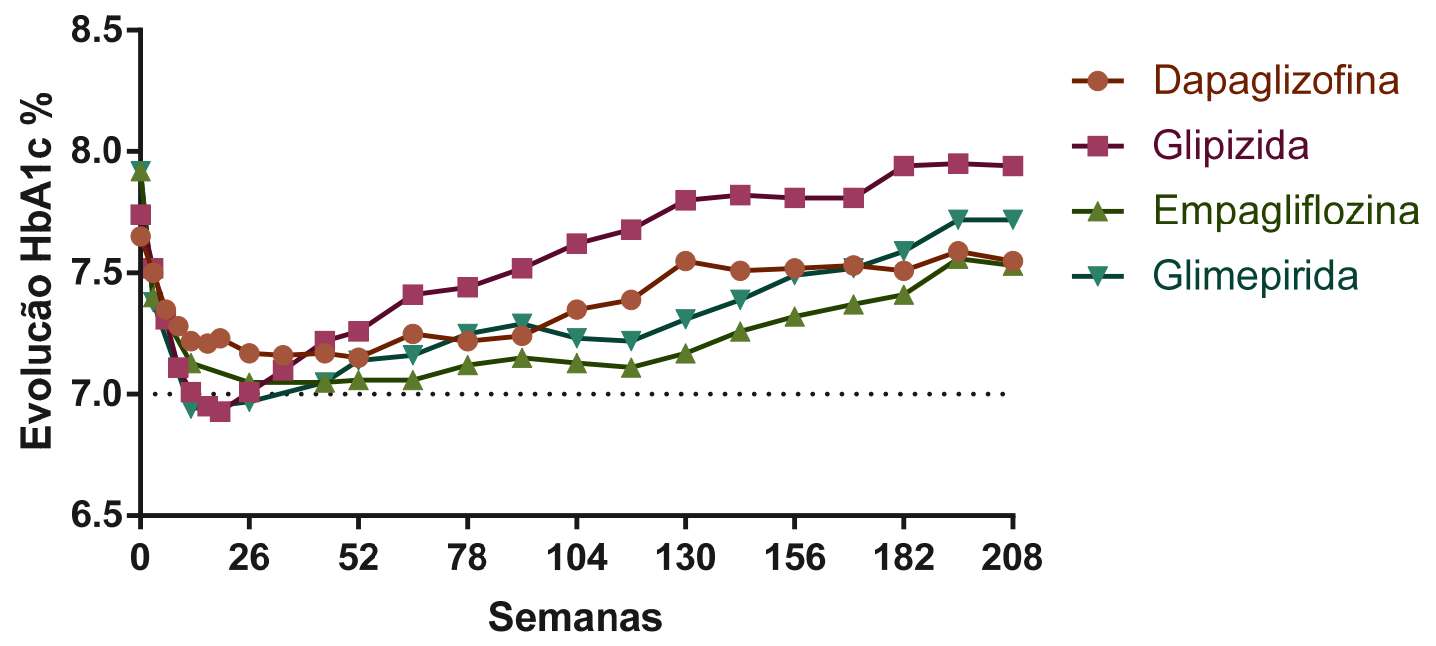

Fonte: resultado da pesquisa

Gráfico 3 - Variação HbA1c em relação ao basal

Variação evolução HbA1c em relação ao basal

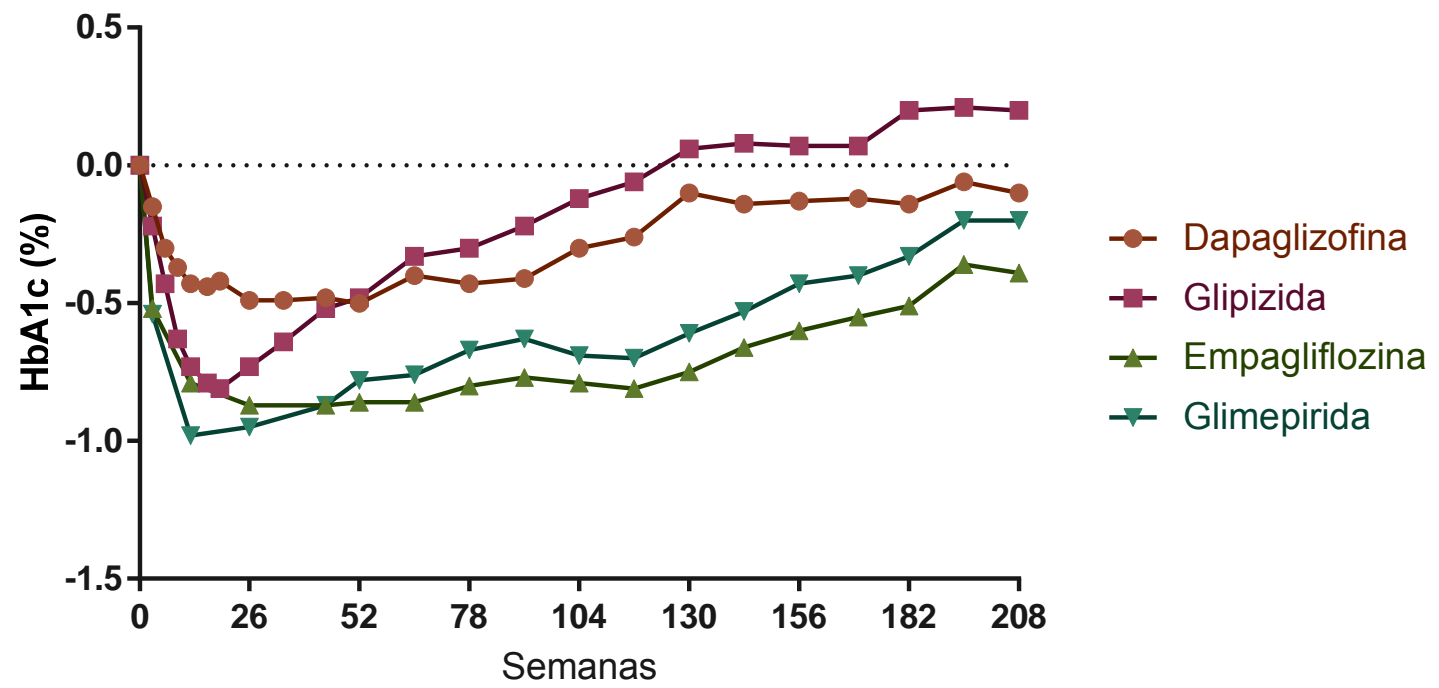

Fonte: resultado da pesquisa 
$\mathrm{Na}$ avaliação dos estudos com quatro anos (maior tempo de segmento), somente o estudo SAXA forneceu o número total de pacientes com $\mathrm{HbA} 1 \mathrm{c} \leq 7 \%$ ao final do estudo, para os estudos DAPA e EMPA foram realizadas extrapolações utilizando-se a média amostral e desvio padrão e são apresentados na Tabela 7.

Tabela 7 - Estimativa de pacientes com $\mathrm{HbA1c} \leq 7 \%$ ao longo do segmento

\begin{tabular}{|c|c|c|c|c|c|c|c|c|c|c|c|c|c|c|c|c|c|c|}
\hline \multirow{6}{*}{$\begin{array}{l}\text { ESTUDO } \\
\text { Tempo } \\
\text { (em } \\
\text { anos) }\end{array}$} & \multicolumn{6}{|c|}{ DAPA } & \multirow{2}{*}{\multicolumn{6}{|c|}{ EMPA }} & \multicolumn{6}{|c|}{ SAXA } \\
\hline & \multirow{3}{*}{\multicolumn{3}{|c|}{$\begin{array}{l}\text { Intervenção } \\
\text { Dapaglifozina }\end{array}$}} & & & & & & & & & & & & & \multirow{3}{*}{\multicolumn{3}{|c|}{$\begin{array}{c}\text { Comparad } \\
\text { or } \\
\text { Placebo }\end{array}$}} \\
\hline & & & & \multirow{2}{*}{\multicolumn{3}{|c|}{$\begin{array}{l}\text { Comparador } \\
\text { Glipizida }\end{array}$}} & \multirow{2}{*}{\multicolumn{3}{|c|}{$\begin{array}{c}\text { Intervenção } \\
\text { Empaglifozina }\end{array}$}} & \multirow{2}{*}{\multicolumn{3}{|c|}{$\begin{array}{c}\text { Comparador } \\
\text { Glimepirida }\end{array}$}} & \multirow{2}{*}{\multicolumn{3}{|c|}{$\begin{array}{l}\text { Intervenção } \\
\text { Saxagliptina }\end{array}$}} & & & \\
\hline & & & & & & & & & & & & & & & & & & \\
\hline & \multicolumn{3}{|c|}{$n \leq$} & \multicolumn{3}{|c|}{$n \leq$} & \multicolumn{3}{|c|}{$\mathrm{n} \leq$} & \multicolumn{3}{|c|}{$\mathrm{n} \leq$} & \multicolumn{3}{|c|}{$\mathrm{n} \leq$} & \multicolumn{3}{|c|}{$\mathrm{n} \leq$} \\
\hline & $\mathrm{n}$ & 7 & (\%) & $\mathrm{n}$ & 7 & (\%) & $\mathrm{n}$ & 7 & (\%) & $n$ & 7 & (\%) & $\mathrm{n}$ & 7 & (\%) & $\mathrm{n}$ & 7 & (\%) \\
\hline & 35 & & 35 & 35 & & 49 & 70 & & 47 & 69 & & 57 & & & & & & \\
\hline \multirow[t]{2}{*}{0,5} & 4 & 141 & $\%$ & 4 & 195 & $\%$ & 2 & 359 & $\%$ & 9 & 441 & $\%$ & - & - & - & - & - & - \\
\hline & 32 & & 35 & 31 & & 34 & 64 & & 43 & 60 & & 40 & & & & & & \\
\hline \multirow[t]{2}{*}{1} & 1 & 138 & $\%$ & 5 & 136 & $\%$ & 5 & 326 & $\%$ & 9 & 309 & $\%$ & - & - & - & - & - & - \\
\hline & 23 & & 21 & 20 & & 14 & 54 & & 36 & 45 & & 26 & & & 23 & & & \\
\hline \multirow[t]{2}{*}{2} & 3 & 82 & $\%$ & 8 & 55 & $\%$ & 5 & 273 & $\%$ & 7 & 201 & $\%$ & - & 43 & $\%$ & - & 16 & $9 \%$ \\
\hline & 10 & & & 10 & & & 41 & & 27 & 36 & & 17 & & & 15 & & & \\
\hline \multirow[t]{2}{*}{3} & 5 & 23 & $6 \%$ & 2 & 14 & $3 \%$ & 3 & 206 & $\%$ & 5 & 132 & $\%$ & - & 29 & $\%$ & - & 9 & $5 \%$ \\
\hline & & & & & & & 36 & & 23 & 24 & & & 5 & & & & & \\
\hline 4 & 79 & 21 & $5 \%$ & 71 & 14 & $3 \%$ & 5 & 178 & $\%$ & 3 & 44 & $6 \%$ & 8 & 14 & $7 \%$ & . & 7 & $4 \%$ \\
\hline
\end{tabular}

Os valores porcentuais são em relação ao número inicial de pacientes em cada grupo. Fonte: resultado da pesquisa

Para complementar a análise de eficácia da meta de HbA1c $\leq 7 \%$, foi realizado o teste do qui-quadrado, da proporção de pacientes em cada braço com $\mathrm{HbA} 1 \mathrm{c} \leq 7$ ao final do estudo em relação ao número inicial da amostra. Os dados são apresentados na Tabela 8. A empagliflozina foi o tratamento mais eficaz quando comparado com dapagliflozina e sitagliptina. 
Tabela 8 - Teste do qui-quadrado: proporção de pacientes com HbA1c $\leq 7$ ao final de quatro anos

\begin{tabular}{|cccccccc}
\multicolumn{2}{c}{ BRAÇOS TERAPÊUTICOS } & \multicolumn{2}{c}{ Tratamento 1 } & \multicolumn{2}{c}{ Tratamento 2 } & & \\
Tratamento 1 & Tratamento 2 & $\mathbf{n}$ & prop & $\mathbf{n}$ & prop & $\mathbf{Q}^{\mathbf{2}}$ & $\mathbf{p}$ \\
dapagliflozina & glipizida & 400 & 0,05 & 401 & 0,03 & 1,09 & 0,296 \\
empagliflozina & glimepirida & 765 & 0,23 & 780 & 0,06 & 96,10 & $<0,001$ \\
sitagliptina & placebo & 191 & 0,07 & 179 & 0,04 & 1,43 & 0,232 \\
dapagliflozina & empagliflozina & 400 & 0,05 & 765 & 0,23 & 58,94 & $<\mathbf{0 , 0 0 1}$ \\
dapagliflozina & sitagliptina & 400 & 0,05 & 191 & 0,07 & 0,67 & 0,415 \\
empagliflozina & sitagliptina & 765 & 0,23 & 191 & 0,07 & 23,21 & $<\mathbf{0 , 0 0 1}$
\end{tabular}

n: número da amostra, prop: proporção final de pacientes na meta, $Q^{2}$ : teste do quiquadrado, p: significância estatística

Fonte: resultado da pesquisa

\subsubsection{Metanálise para HbA1c}

Foram analisados os dados de $\mathrm{HbA} 1 \mathrm{c}$ referentes ao maior tempo de segmento (quatro anos). Optou-se por utilizar os valores iniciais e finais de $\mathrm{HbA1c}$ e não a variação em relação ao basal. Na Tabela 9 encontram-se o resumo das variáveis e no Gráfico 4 a representação da metanálise. 
Tabela 9 - Metanálise para HbA1c (\%) em quatro anos nos estudos DAPA e EMPA

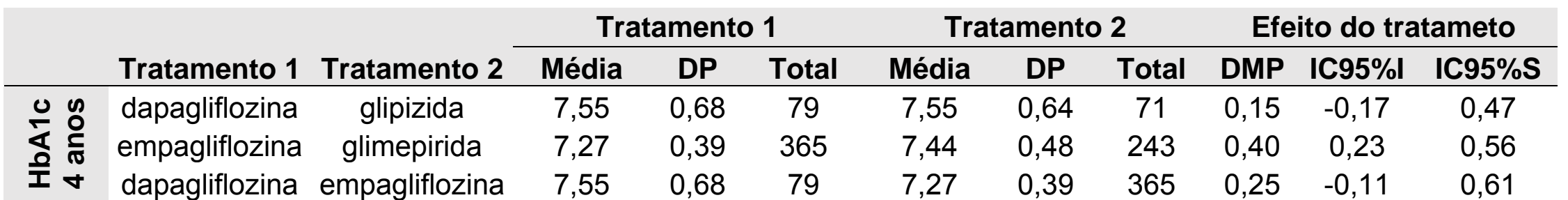

DP: desvio padrão, DMP: diferença média padronizada, IC95\%I: 95\% do intervalo de confiança inferior, IC95\%S: $95 \%$ do intervalo de confiança superior

\section{Gráfico 4 - Representação gráfica da metanálise para HbA1c em quatro anos}

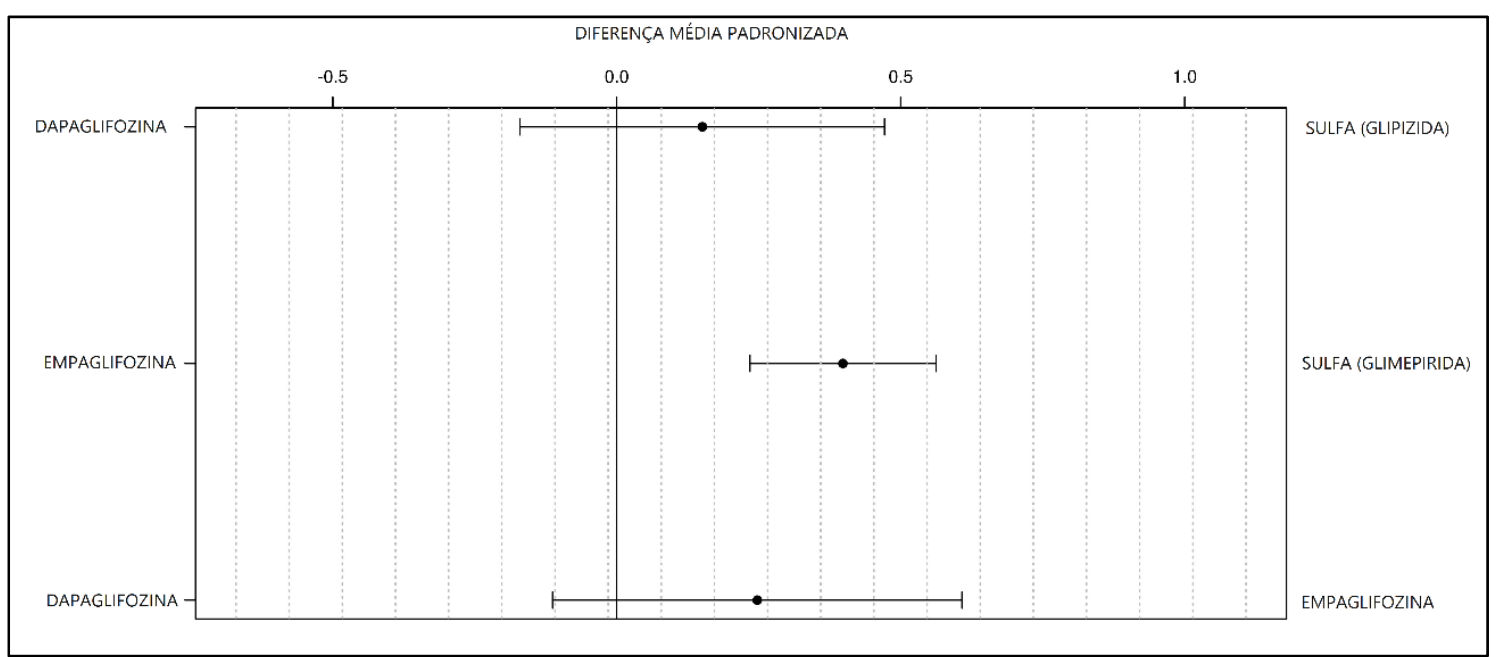

Fonte: resultado da pesquisa 
Na comparação do estudo DAPA (dapagliflozina versus glipizida), a variação da HbA1c não apresentou significância estatística. Para o estudo EMPA (empagliflozina versus glimepirida) houve uma redução significativa da HbA1c (DMP $=0,40$, IC $95 \%$ 0,23- 0,56). Na meta comparação entre dapagliflozina e empagliflozina não houve significância estatística.

Pela escassa quantidade de estudos não foi possível medir a heterogeneidade e realizar a análise de sensibilidade.

\subsubsection{Revisão sistemática em relação à variação do peso}

O estudo DAPA não fez referência ao peso inicial dos pacientes no início do estudo e inclui dados de pacientes após o resgate. O estudo SAXA não disponibiliza os dados relativos ao peso. Na Tabela 10, se observa a evolução do peso ao longo dos estudos. O Gráfico 5 foi confeccionado com os resultados de peso e os dados faltantes digitalizados dos estudos. 
Tabela 10 - Variação do peso $(\mathrm{Kg})$ nos estudos

\begin{tabular}{|c|c|c|c|c|c|c|c|c|c|c|c|c|}
\hline \multirow{4}{*}{$\begin{array}{c}\text { ESTUDO } \\
\text { Tempo } \\
\text { (em anos) }\end{array}$} & \multicolumn{6}{|c|}{ DAPA } & \multicolumn{6}{|c|}{ EMPA } \\
\hline & \multirow{2}{*}{\multicolumn{3}{|c|}{$\begin{array}{l}\text { Intervenção } \\
\text { Dapaglifozina }\end{array}$}} & \multirow{2}{*}{\multicolumn{3}{|c|}{$\begin{array}{c}\text { Comparador } \\
\text { Glipizida }\end{array}$}} & \multirow{2}{*}{\multicolumn{3}{|c|}{$\begin{array}{l}\text { Intervenção } \\
\text { Empaglifozina }\end{array}$}} & \multirow{2}{*}{\multicolumn{3}{|c|}{$\begin{array}{l}\text { Comparador } \\
\text { Glimepirida }\end{array}$}} \\
\hline & & & & & & & & & & & & \\
\hline & $\mathrm{n}$ & Média & $\mathrm{DP}$ & $\mathrm{n}$ & Média & DP & $\mathrm{n}$ & Média & DP & $\mathrm{n}$ & Média & DP \\
\hline 0,5 & - & $-3,7$ & - & - & 1,3 & - & 706 & $-3,3$ & 2,6 & 703 & 1,5 & 2,8 \\
\hline 1 & 323 & $-3,2$ & 3,2 & 315 & 1,4 & 3,1 & 643 & $-2,9$ & 2,6 & 610 & 1,4 & 2,5 \\
\hline 2 & 234 & $-3,7$ & 3,9 & 211 & 1,4 & 3,9 & 555 & $-2,8$ & 2,4 & 462 & 1,1 & 3,3 \\
\hline 3 & - & $-4,0$ & - & - & 1,1 & - & 443 & $-3,7$ & 1,9 & 301 & 0,9 & 1,8 \\
\hline 4 & 159 & $-3,7$ & 4,1 & 140 & 0,7 & 4,0 & 336 & $-4,1$ & 1,9 & 248 & 0,8 & 1,8 \\
\hline
\end{tabular}

O estudo DAPA incluiu em sua análise dados de peso de pacientes após o resgate e a metodologia LOCF (last observation caried forward), estudo EMPA utilizou MMRM (mixed effect model repeat measurement). Fonte: resultado da pesquisa

\section{Gráfico 5 - Evolução da variação de peso nos estudos DAPA e EMPA}

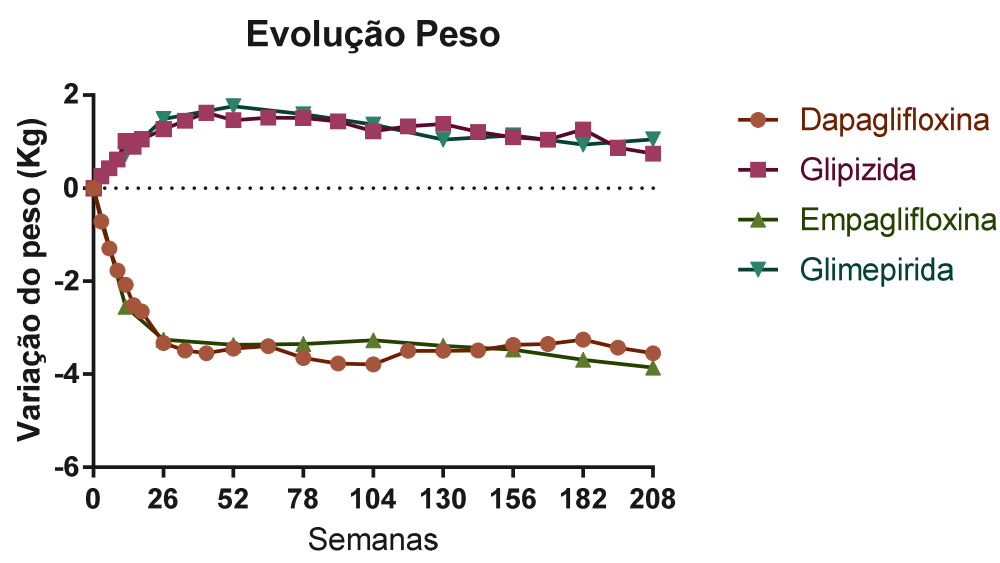

Fonte: resultado da pesquisa 


\subsubsection{Metanálise em relação à variação do peso}

Foram analisados os dados referentes à variação do peso basal no maior tempo de segmento (quatro anos). Na Tabela 11 encontram-se o resumo das variáveis e no Gráfico 6 a representação da metanálise. 
Tabela 11 - Metanálise da variação de peso $(\mathrm{Kg})$ em relação ao basal em quatro anos nos estudos DAPA e EMPA

\begin{tabular}{|c|c|c|c|c|c|c|c|c|c|c|c|}
\hline \multirow{5}{*}{ 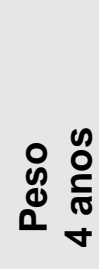 } & \multirow[b]{2}{*}{ Tratamento 1} & \multirow[b]{2}{*}{ Tratamento 2} & \multicolumn{3}{|c|}{ Tratamento 1} & \multicolumn{3}{|c|}{ Tratamento 2} & \multicolumn{3}{|c|}{ Efeito do tratameto } \\
\hline & & & Média & DP & Total & Média & DP & Total & DMP & IC95\%I & IC95\%S \\
\hline & dapagliflozina & glipizida & $-3,65$ & 4,12 & 159 & 0,73 & 4,04 & 140 & 1,07 & 0,83 & 1,32 \\
\hline & empagliflozina & glimepirida & $-4,06$ & 1,87 & 336 & 0,82 & 1,84 & 248 & 2,63 & 2,40 & 2,85 \\
\hline & dapagliflozina & empagliflozina & $-3,65$ & 4,12 & 159 & $-4,06$ & 1,87 & 336 & 1,56 & 1,23 & 1,88 \\
\hline
\end{tabular}

DP: desvio padrão, DMP: diferença média padronizada, IC95\%l: 95\% do intervalo de confiança inferior, IC $95 \%$ S: $95 \%$ do intervalo de confiança superior

Fonte: resultado da pesquisa

\section{Gráfico 6 - Representação gráfica da metanálise de variação do peso em relação ao basal em quatro anos}

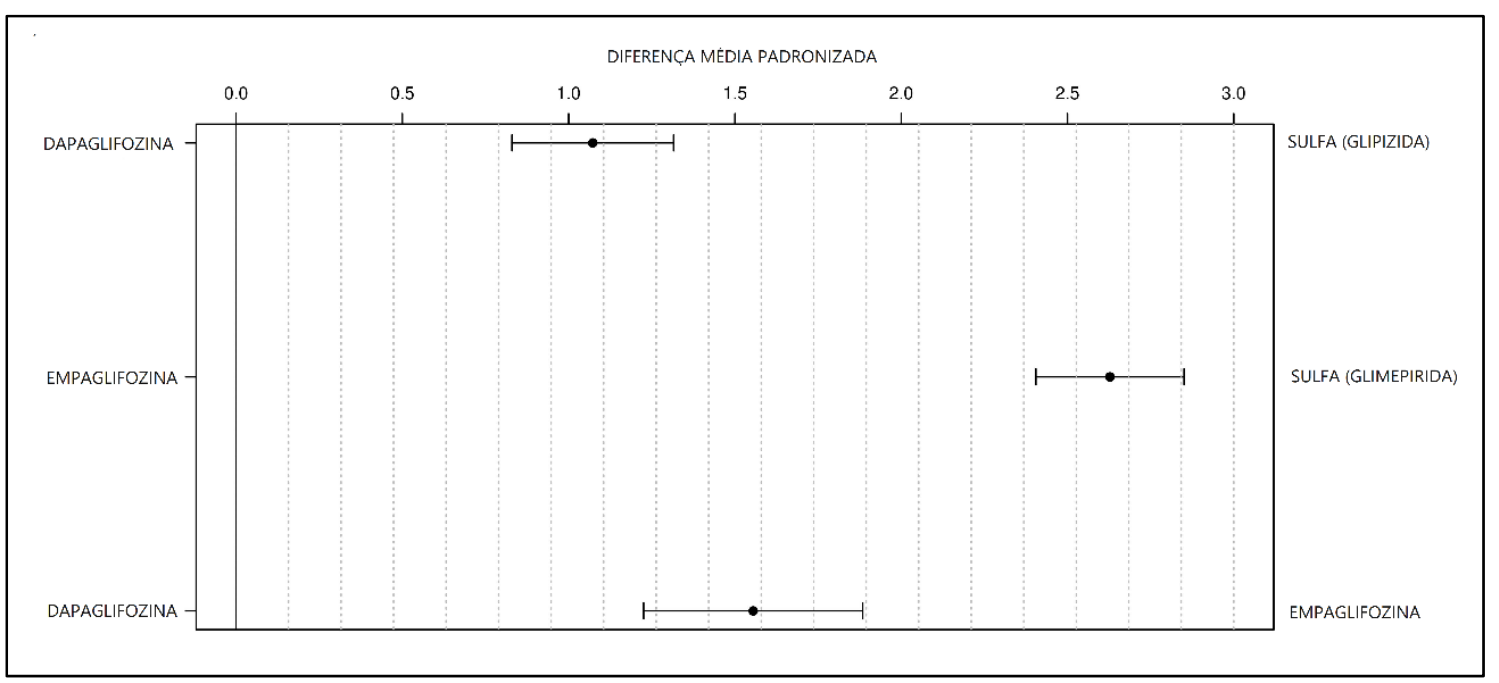

Fonte: resultado da pesquisa 
Na comparação do estudo DAPA (dapagliflozina versus glipizida), a variação do peso apresentou significância estatística, favorecendo a dapagliflozina (DMP = 1,07, IC 95\% 0,83-1,32). Para o estudo EMPA (empagliflozina versus glimepirida) a análise também demonstrou significância estatística para a empagliflozina (DMP = 2,63, IC 95\% 2,40-2,85). Na meta comparação entre dapagliflozina e empagliflozina houve significância estatística favorecendo a empagliflozina (DMP = 1,56, IC 95\% 1,22- 1,88).

Pela escassa quantidade de estudos não foi possível medir a heterogeneidade e realizar a análise de sensibilidade.

\subsubsection{Revisão sistemática em relação à frequência de pacientes com hipoglicemia}

Houve uma variação da definição de hipoglicemia entre os estudos (Anexo I). $\mathrm{Na}$ Tabela 12 se observa a frequência de pacientes com eventos de hipoglicemia por estudo. $\mathrm{O}$ Gráfico 7 foi confeccionado com a frequência percentual de pacientes com hipoglicemia nos estudos com duração de quatro anos. 
Tabela 12 - Frequência de pacientes com eventos de hipoglicemia por períodos de tempo

\begin{tabular}{|c|c|c|c|c|c|c|c|c|c|c|c|c|}
\hline \multirow{2}{*}{ Estudo } & \multicolumn{4}{|c|}{ DAPA } & \multicolumn{4}{|c|}{ EMPA } & \multicolumn{4}{|c|}{ SAXA } \\
\hline & \multicolumn{2}{|c|}{ Intervenção } & \multicolumn{2}{|c|}{ Comparador } & \multicolumn{2}{|c|}{ Intervenção } & \multicolumn{2}{|c|}{ Comparador } & \multicolumn{2}{|c|}{ Intervenção } & \multicolumn{2}{|c|}{ Comparador } \\
\hline Tempo em & Dapaflc & zina & Glipizi & & Empaglif & ozina & Glimepi & rida & Saxaglip & tina & Place & \\
\hline intervalo & Eventos & $n$ & Eventos & $n$ & Eventos & $n$ & Eventos & $n$ & Eventos & $n$ & Eventos & $n$ \\
\hline 6 meses & - & - & - & - & - & 765 & - & 780 & $11(5,7)$ & 191 & $10(5,6)$ & 179 \\
\hline $6 \leq 1$ ano & $14(3)$ & 406 & $162(40)$ & 408 & $12(2)$ & - & $159(20)$ & - & - & - & - & - \\
\hline $1 \leq 2$ anos & $5(2)$ & 315 & $73(24)$ & 309 & $19(2)$ & - & $189(24)$ & - & - & - & - & - \\
\hline $2 \leq 3$ anos & $3(2)$ & 204 & $70(37)$ & 188 & - & - & - & - & - & - & - & - \\
\hline $3 \leq 4$ anos & $4(2)$ & 178 & $46(29)$ & 162 & - & - & - & - & - & - & - & - \\
\hline Total & $22(5)$ & 406 & $210(52)$ & 408 & $24(3)$ & 765 & $218(28)$ & 780 & $22(12)$ & 191 & $21(12)$ & 179 \\
\hline
\end{tabular}

Porcentagem entre parênteses. Fonte: resultado da pesquisa

Gráfico 7 - Representação gráfica da frequência de pacientes com eventos de hipoglicemia

Frequência percentual de pacientes com eventos de hipoglicemia

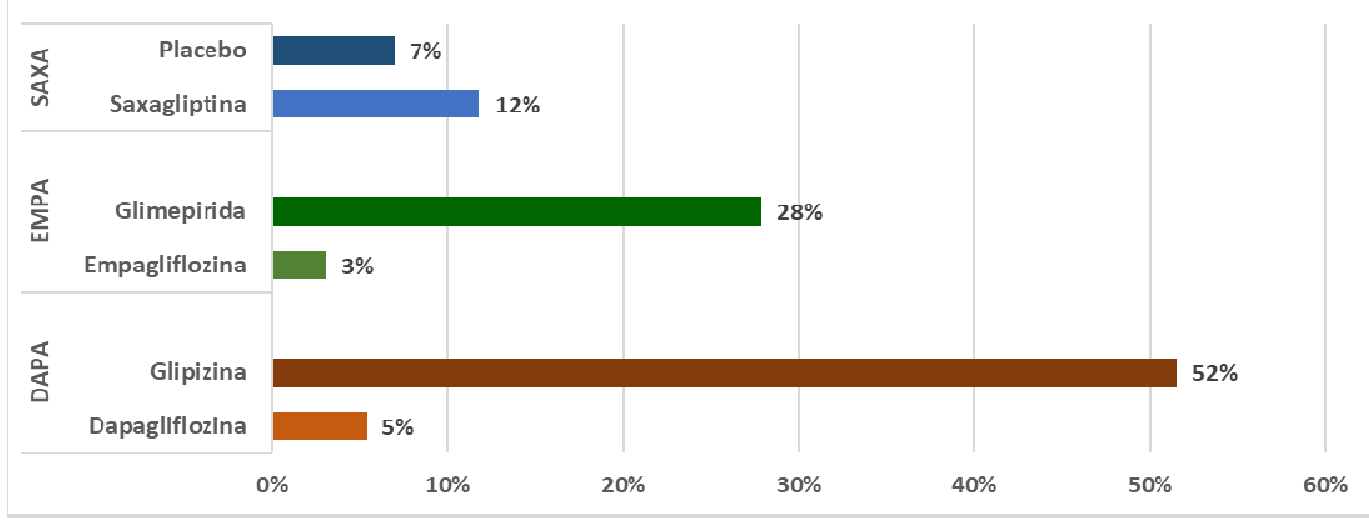

Fonte: resultado da pesquisa 


\subsubsection{Metanálise da frequência de pacientes com hipoglicemia}

Foram analisados os dados referentes à frequência de pacientes com eventos de hipoglicemia durante todo o período de segmento dos estudos DAPA e EMPA. Na Tabela 13, encontra-se o resumo da metanálise e no Gráfico 8, a representação gráfica. 
Tabela 13 - Metanálise para hipoglicemia em quatro anos nos estudos DAPA e EMPA

\begin{tabular}{|c|c|c|c|c|c|c|c|c|c|c|c|}
\hline \multirow{5}{*}{ 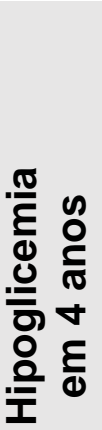 } & \multirow[b]{2}{*}{ Tratamento 1} & \multirow[b]{2}{*}{ Tratamento 2} & \multicolumn{3}{|c|}{$\begin{array}{c}\text { Tratamento } 1 \\
\text { N de }\end{array}$} & \multicolumn{3}{|c|}{ Tratamento 2} & \multicolumn{3}{|c|}{ Efeito do tratameto } \\
\hline & & & Prop & casos & Total & Prop & N de casos & Total & RC & IC95\%I & IC95\%S \\
\hline & dapagliflozina & glipizida & 0,05 & 22 & 406 & 0,51 & 210 & 408 & 0,05 & 0,03 & 0,09 \\
\hline & empagliflozina & glimepirida & 0,03 & 24 & 765 & 0,28 & 218 & 780 & 0,08 & 0,05 & 0,13 \\
\hline & dapagliflozina & empagliflozina & 0,05 & 22 & 406 & 0,03 & 24 & 765 & 1,53 & 0,80 & 2,91 \\
\hline
\end{tabular}

Prop: frequência de pacientes com hipoglicemia, N de casos: número de casos, RC: razão de chances (odds ratio), IC95\%l: 95\% do intervalo de confiança inferior, IC95\%S: 95\% do intervalo de confiança superior

Fonte: resultado da pesquisa

Gráfico 8 - Representação gráfica da metanálise de frequência de pacientes com hipoglicemia nos estudos DAPA e EMPA

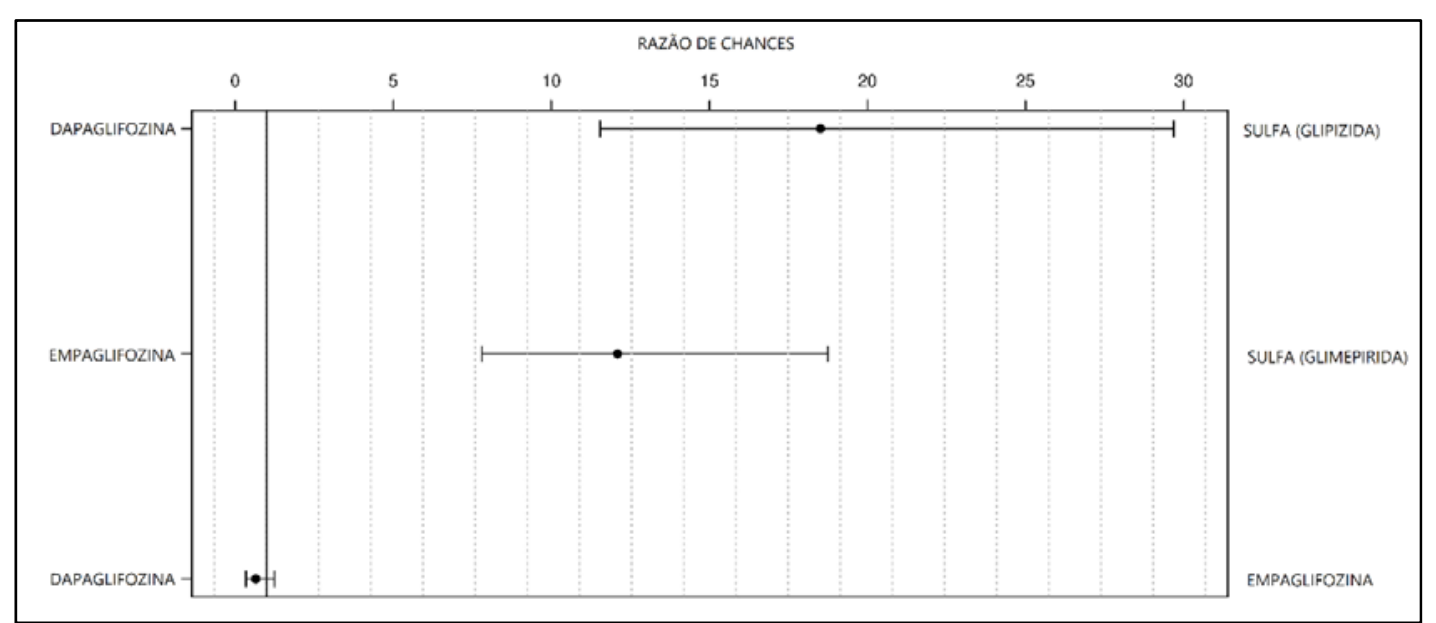

Fonte: resultado da pesquisa 
$\mathrm{Na}$ comparação do estudo DAPA (dapagliflozina versus glipizida), a frequência de pacientes com hipoglicemia foi significativamente maior nos pacientes em uso de glipizida ( $R C=0,05$, IC 95\% 0,03- 0,09). Para o estudo EMPA (empagliflozina versus glimepirida) a análise também demonstrou uma frequência de pacientes com hipoglicemia maior no braço glimepirida $(R C=0,08, I C 95 \% 0,05-$ 0,13). Na meta comparação entre dapagliflozina e empagliflozina não houve significância estatística. A diferença média entre os valores basais e quatro anos de segmento para dapagliflozina, empagliflozina e sulfoniluréias foi de $-0,23,-0,92$ e $0,41 \%$ (respectivamente).

Pela escassa quantidade de estudos não foi possível medir a heterogeneidade e realizar a análise de sensibilidade.

\subsubsection{Resultados da metanálise - subgrupos}

Por falta de dados, as análises referentes à idade, tempo de diagnóstico e sexo não foram realizadas.

\subsubsection{Risco de vieses dos estudos incluídos}

As características metodológicas dos ensaios clínicos randomizados incluídos foram avaliadas individualmente.

Avaliou-se a metodologia adequada de randomização, a descrição do sigilo da lista de alocação, se existiu algum nível de cegamento e se as análises seguiram o princípio de intenção de tratar.

A Tabela 14 resume os achados. De uma maneira geral, os estudos incluídos descrevem as características metodológicas utilizadas e não há limitações sérias entre os estudos. 
Tabela 14 - Risco de viés baseado nas características metodológicas dos estudos incluídos

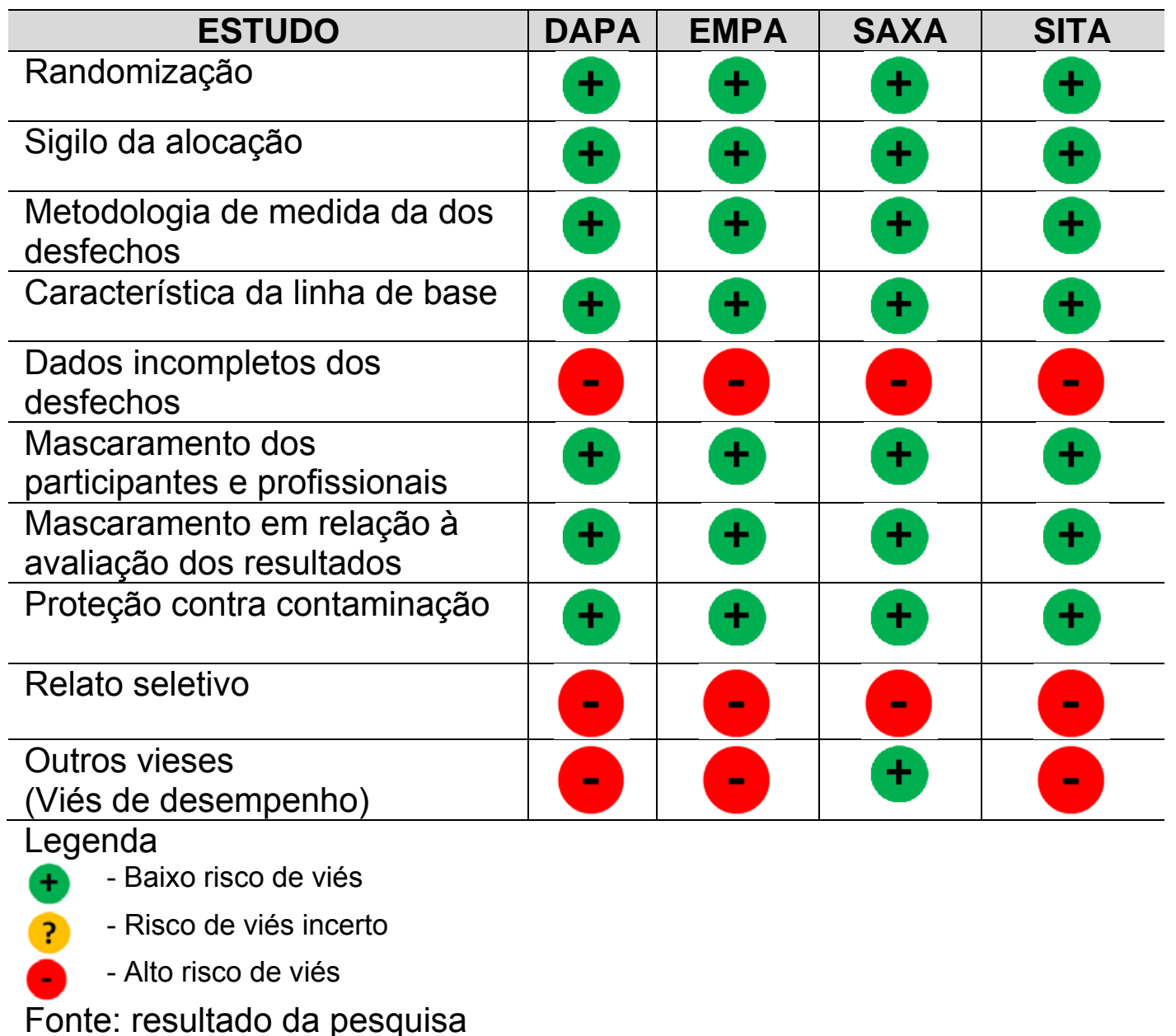

\subsubsection{Qualidade da evidência}

A qualidade da evidência foi avaliada para os desfechos $\mathrm{HbA} 1 \mathrm{c}$, variação de peso e hipoglicemia adaptada da ferramenta GradePro para uma metanálise em rede. A Tabela 15 apresenta os resultados. As notas de rodapé justificam o decréscimo da graduação da qualidade. 


\section{Tabela 15 - Qualidade da evidência}

Qual é a melhor opção teraupêutica após a falência primária com metformina em longo prazo?

Pacientes: DM2 com falência primária de metformina

Intervenção: dapagliflozina e empagliflozina

Comparador: sulfoniluréias (glipizida e glimepirida)

\begin{tabular}{|c|c|c|c|c|c|c|}
\hline \multirow[t]{2}{*}{ Desfechos } & \multicolumn{6}{|c|}{ Diferença média ou razão de chances e intervalo de confiança. } \\
\hline & \multicolumn{2}{|l|}{ Dapagliflozina } & \multicolumn{2}{|l|}{ Empagliflozina } & \multirow{3}{*}{$\begin{array}{l}\text { Sulfoniluréias } \\
-0,4^{2}\end{array}$} & \multirow{3}{*}{$\begin{array}{l}\text { Importância } \\
\text { Crítica }\end{array}$} \\
\hline $\mathrm{HbA1c}(\%)$ & DM 0,2 & $-0,2^{2}$ & DM 0,4 & $-0,9^{2}$ & & \\
\hline & $(-0,2-0,5)$ & $(-0,0-0,4)$ & $(0,2-0,6)$ & $(-0,4--0,5)$ & & \\
\hline \multirow{4}{*}{$\begin{array}{l}\mathrm{HbA} 1 \mathrm{c}<7 \% \text { em } \\
4 \text { anos }\end{array}$} & \multicolumn{2}{|l|}{$5 \%$} & \multicolumn{2}{|l|}{$23 \%$} & \multirow[t]{4}{*}{$5 \%$ (2 estudos) } & \\
\hline & \multicolumn{2}{|c|}{$\oplus \bigcirc \bigcirc \bigcirc$ MUITO BAIXA ${ }^{3}$} & \multicolumn{2}{|c|}{$\oplus \bigcirc \bigcirc \bigcirc$ MUITO BAIXA $^{3}$} & & \\
\hline & \multicolumn{2}{|c|}{ Confidência estimada no risco relativo } & \multicolumn{2}{|c|}{ Confidência estimada no risco relativo } & & \\
\hline & \multicolumn{2}{|c|}{ Baeado em 801 pacientes (1 estudo) } & \multicolumn{2}{|c|}{ Baeado em 1.541 pacientes (1 estudo) } & & \\
\hline Variação do peso & DM 1,1 & $-3,6^{2}$ & DM 2,6 & $-4,1^{2}$ & $0,8^{2}$ & Crítica \\
\hline \multirow[t]{4}{*}{$(\mathrm{Kg})$} & $(0,8-1,3)$ & $(-4,3--3,0)$ & $(2,4-2,8)$ & $(-4,1--3,9)$ & $(-2,3--1,3)$ & \\
\hline & \multicolumn{2}{|c|}{$\oplus \bigcirc \bigcirc \bigcirc$ MUITO BAIXA ${ }^{3}$} & \multicolumn{2}{|c|}{$\oplus \oplus \bigcirc \bigcirc$ BAIXA } & & \\
\hline & \multicolumn{2}{|c|}{ Confidência estimada no risco de vies } & \multicolumn{2}{|c|}{ Confidência estimada no risco de viés } & & \\
\hline & \multicolumn{2}{|c|}{ Baseado em 801 pacientes ( 1 estudo) } & \multicolumn{2}{|c|}{ Baeado em 1.541 pacientes (1 estudo) } & & \\
\hline Hipoglicemia & RC 0,05 & $\begin{array}{l}30 \text { a menos } \\
\text { em } 100\end{array}$ & RC 0,08 & $\begin{array}{l}33 \text { a menos } \\
\text { em } 100\end{array}$ & 36 em 100 & Crítica \\
\hline \multirow[t]{3}{*}{$\begin{array}{l}\text { (Frequência de } \\
\text { pacientes com } \\
\text { hipoglimia) }\end{array}$} & $(0,03-0,09)$ & $\begin{array}{l}(28 \text { a } 33 \text { a } \\
\text { menos) }\end{array}$ & $(0,05-0,13)$ & $\begin{array}{l}(31 \text { a } 34 \text { a } \\
\text { menos) }\end{array}$ & & \\
\hline & \multicolumn{2}{|c|}{$\oplus \oplus \oplus \bigcirc$ MODERADA $^{5}$} & \multicolumn{2}{|c|}{$\oplus \oplus \oplus \bigcirc$ MODERADA $^{5}$} & & \\
\hline & \multicolumn{2}{|c|}{ Confidência estimada no risco de vies } & \multicolumn{2}{|c|}{ Confidência estimada no risco de viés } & & \\
\hline
\end{tabular}




\section{Baseado em 801 pacientes (1 estudo) Baeado em 1.541 pacientes (1 estudo)}

DM: diferença média, IC: intervalo de confiança, RC: razão de chances (odds ratio)

Avalição do GRADE

Alta qualidade: é improvável que outras pesquisas mudem a confiança na estimativa de efeito.

Qualidade moderada: é provável que outras pesquisas tenham um impacto importante na confiança da estimativa de efeito e podem alterar a estimativa.

Baixa qualidade: é muito provável que outras pesquisas tenham um impacto importante na confiança da estimativa de efeito e é provável que alterem a estimativa.

Qualidade muito baixa: há muita incerteza sobre a estimativa.

${ }^{1}$ Sem dados suficientes para a comparação com saxagliptina

${ }^{2} \mathrm{Em}$ relação ao valor basal

${ }^{3}$ Baseado em: risco de viés não grave, inconsistência muito grave, evidência indireta não grave e imprecisão grave.

${ }^{4}$ Baseado em: risco de viés não grave, inconsistência grave, evidência indireta não grave e imprecisão grave.

5 Baseado em: risco de viés não grave, inconsistência grave, evidência indireta não grave e imprecisão grave.

Fonte: resultado da pesquisa 


\section{DISCUSSÃO}

A literatura médica ainda é carente de estudos randomizados para avaliar a eficácia dos medicamentos para diabetes mellitus tipo 2 após a falência da monoterapia com metformina em longo prazo, os três estudos com maior duração encontrados tiveram duração de quatro anos após randomização (DAPA, EMPA e SAXA). Nesta revisão, foram encontrados poucos estudos com segmento maior que dois anos, sendo que outros artigos já descreveram os efeitos terapêuticos das gliptinas e gliflozinas em um período menor ou igual a dois anos $(77,127,128)$. Esta é a primeira revisão a avaliar um período de quatro anos de segmento das gliflozinas e gliptinas após falência do tratamento primário com metformina.

Todos os estudos fizeram referência a pelo menos uma orientação inicial sobre dieta e atividade física. Estratégias bem sucedidas de orientação dietética e atividade física podem provocar uma perda de peso de 5 a $8 \%$ do peso corporal total (129), que pode ter um grande impacto no controle glicêmico em longo prazo (130). Entretanto, os estudos selecionados não demonstram uma preocupação com este tema e suas consequências em longo prazo.

Muitos dos dados referentes à falência medicamentosa em longo prazo são derivados de coortes populacionais (70). O único ensaio clínico randomizado de longo prazo, que teve duração de 20 anos, em pacientes com diabetes mellitus tipo 2 foi o UK Prospective Diabetes Study (UKPDS), financiado pelo governo britânico no período de 1977 a 1997 (12,131). Este estudo foi um marco no tratamento do DM2, suas conclusões indicaram que o tratamento medicamentoso é progressivo, geralmente necessitando de várias drogas em longo prazo (132). Este estudo também consagrou a superioridade da metformina como tratamento inicial em relação à insulina e sulfoniluréias, devido à manutenção do peso e baixo risco de hipoglicemias (131).

As recomendações clínicas da American Diabetes Association, American Association of Clinical Endocrinologists e Sociedade Brasileira de Diabetes sugerem como droga inicial a metformina caso não haja contraindicações $(18,133,134)$, porém a escolha da segunda droga leva em consideração uma série de fatores, não havendo uma droga de escolha, porém os consensos não consideram o tempo de 
falência da terapia. Portanto, se fazem necessários estudos randomizados, revisões sistemáticas e metanálises que avaliem a eficácia dessas medicações e inclusão nas recomendações clínicas como um fator importante de escolha de uma segunda terapia a manutenção do controle glicêmico em longo prazo.

Os estudos selecionados nesta revisão foram patrocinados por laboratórios farmacêuticos e como critério de inclusão principal foram elegidos pacientes com DM2 em falência primária de metformina $(\geq 1.500 \mathrm{mg} / \mathrm{d})$ com $\mathrm{HbA} 1 \mathrm{c}$ de entrada variando de 6,5 a 10\%. Com exceção do estudo DAPA, que permitiu a retirada de um antidiabético oral antes do período de randomização, com subsequente ajuste da dose de metformina (116). Na abordagem mais moderna, alguns consensos já preconizam a terapia dupla inicial com $\mathrm{HbA} 1 \geq 7,5 \%$ (133), devido ao risco cardiovascular aumentado em até $26 \%$ em cinco anos, na demora do controle inicial da glicemia no primeiro ano após o diagnóstico (28). Em nossa revisão, a HbA1c basal variou de 7,6 a $8,2 \%$. Um estudo de 2015, sugeriu que a terapia tripla em paciente com diabetes recém diagnosticados, se mostrou mais eficaz para redução da HbA1c e apresentou menos hipoglicemias que a adição progressiva da terapia tripla em dois anos de seguimento (135). É possível que o tempo perdido até a falência da terapia primária, esteja relacionado a uma perda significativa da função das células $\beta$, nos estudos avaliados.

Os valores de HbA1c basal para o estudo DAPA foram menos elevados, quando comparados ao estudo EMPA em aproximadamente 0,3\%, apesar de ser uma diferença pequena e sem significância estatística, quando se observa a evolução da HbA1c no tempo, partindo do valores iniciais de $\mathrm{HbA} 1 \mathrm{c}$, os braços dapagliflozina e empagliflozina se aproximam em termos de controle da HbA1c em quatro anos, porém, quando os mesmos valores são comparados em relação a variações em relação aos níveis basais de $\mathrm{HbA1c}$, o braço glimepirida se interpõe entre os braços empagliflozina e dapagliflozina, demonstrando que, além do perfil da droga, o valor inicial de HbA1c pode ser um preditor importante do controle em longo prazo (136). Por esta razão, se optou por trabalhar com os dados reais e não variações do basal. $\mathrm{Na}$ comparação entre os valores finais de $\mathrm{HbA} 1 \mathrm{c}$, a empagliflozina apresentou valores estatisticamente mais baixos que a dapagliflozina, duas explicações são possíveis, a primeira é que a medicação é mais potente e a 
segunda é que houve um escalonamento da dose da dapagliflozina ao longo do estudo, não tendo seu efeito terapêutico máximo desde o início do estudo.

Os estudos DAPA e EMPA utilizaram como comparadores sulfoniluréias (glipizida e glimepirida), permitindo a titulação até metade da dose máxima. São limitadas na literatura as comparações entre sulfoniluréias (137), e algumas metanálises em rede usam as drogas desta classe como um meta comparador único $(128,138)$. A curva de evolução da $\mathrm{HbA} 1 \mathrm{c}$ das sulfoniluréias foi semelhante à das gliflozinas. Somente $63 \%$ dos pacientes no braço glipizida receberam a dose máxima do estudo de $20 \mathrm{mg}$ (110). Não existem dados de quatro anos referentes ao braço glimepirida do estudo EMPA, porém na publicação de dois anos, somente $40 \%$ dos pacientes do grupo glimepirida utilizaram a dose máxima permitida no estudo de $4 \mathrm{mg}$, enquanto as doses de 1, 2 e $3 \mathrm{mg}$ foram utilizadas por 26, 16 e 17\% da população, respectivamente (91). Em um estudo que avaliou a canagliflozina após a falência primária com metformina, não incluído nesta revisão por ter uma duração de dois anos, a titulação de glimepirida foi realizada até a dose máxima recomendada de 8 mg/d (139). Apesar de algumas publicações na literatura indicarem que a potência entre meia dose e dose cheia de sulfoniluréias teriam efeitos semelhantes $(137,140)$, não se pode caracterizar falência terapêutica das sulfoniluréias nos estudos avaliados, em pacientes que não chegam à meia dose preconizada da medicação. Além de que a hipoglicemia pode ter sido um fator limitante na titulação das drogas.

$\mathrm{Na}$ superposição das curvas de evolução da HbA1c nos estudos DAPA e EMPA, percebe-se que em menos de um ano a média da $\mathrm{HbA} 1 \mathrm{c}$ já se encontrava maior que $7 \%$, ou seja, a eficácia terapêutica não é adequada em longo prazo, compatível com outros estudos de coorte para outras classes antidiabéticas (70, 141), assim como para a canagliflozina no estudo Canvas (142). Os estudos selecionados fazem referência ao valor basal, porém os valores reais demonstram descontrole glicêmico precoce. A extrapolação das curvas da dapagliflozina e empagliflozina neste estudo mostram que em um ano apenas 35 e $43 \%$, e em quatro anos, 5 e 23\% teriam $\mathrm{HbA} 1 \mathrm{c} \leq 7 \%$, respectivamente. Esses dados sugerem que $\mathrm{o}$ controle glicêmico já se torna ineficiente em menos de um ano de segmento na maioria dos pacientes. O estudo SAXA forneceu o número de pacientes em 
saxagliptina $5 \mathrm{mg}$ com $\mathrm{HbA} 1 \mathrm{c} \leq 7 \%$ com dois e quatro anos de segmento (23 e 7\%), corroborando o mesmo achado, tanto para as gliflozinas, quanto para a saxagliptina. No estudo que avaliou canagliflozina após falência da metformina após dois anos, o perfil de evolução é semelhante ao encontrado para dapagliflozina e empagliflozina (139).

Quando comparados valores finais de $\mathrm{HbA} 1 \mathrm{c}$, o controle glicêmico foi semelhante nos dois braços do estudo DAPA (dapagliflozina versus gliclazida), diferente dos achados dos autores, já no estudo EMPA, a empagliflozina obteve uma resposta melhor em relação à glimepirida. Da meta comparação entre as duas gliflozinas, não houve diferença significante, levando em consideração os critérios para resgate e titulação da droga de cada estudo. A análise apresenta qualidade da evidência baixa, devido à inconsistência grave (heterogeneidade que não pôde ser calculada) e à imprecisão grave (somente dois estudos). A manutenção dos níveis de HbA1c é "crítica" para o acompanhamento em longo prazo da doença $(18,132)$.

Em menos de um ano de tratamento houve uma estabilização do peso nos estudos DAPA e EMPA nos quatro braços. As duas gliflozinas tiveram uma resposta melhor em relação à variação do peso em relação às sulfoniluréias, sendo que na meta comparação houve superioridade da empagliflozina em relação à dapagliflozina. Estes dados devem ser analisados considerando possíveis confundidores como a titulação progressiva da dapagliflozina, a metodologia utilizada para dados incompletos de cada estudo (LOCF versus MMRM) e que no estudo DAPA os resultados da variação de peso incluem pacientes após o resgate terapêutico, o que dependendo da escolha poderia influenciar o resultado de maneira positiva ou negativa. A análise apresenta qualidade da evidência baixa, devido à inconsistência grave (heterogeneidade que não pôde ser calculada, mas provavelmente alta) e à imprecisão grave (somente dois estudos).

A hipoglicemia é uma preocupação frequente no tratamento do diabetes, especialmente em pacientes em uso de sulfoniluréias, nesta revisão ficou comprovada a baixa frequência de pacientes com hipoglicemia na classe das gliflozinas (dapagliflozina $5 \%$ e empagliflozina $3 \%$ ) em relação a das sulfoniluréias (glipizida 52\% e glimepirida 28\%). Este resultado deve ser avaliado levando em consideração que cada estudo teve critérios próprios de hipoglicemia objetivos e 
subjetivos. Na meta comparação não houve diferença estatisticamente significante entre dapagliflozina e empagliflozina. A análise apresenta qualidade de evidência moderada, devido à inconsistência grave (métodos de medida diferentes), evidência indireta não grave e uma imprecisão grave (somente dois estudos).

Os dados dos ensaios clínicos randomizados foram apresentados de forma diferente, o que pode ter impactactado na análise dos resultados. No estudo DAPA, para as análises dos dados faltantes foi utilizada a estratégia last observation carried forward (LOCF), no estudo EMPA mixed model for repeated measures (MMRM), já no estudo SITA a estratégia foi observed case (OC). Cada método faz inferências relativas às características dos dados faltantes. Se os pressupostos para qualquer método particular não são válidos, os resultados desse método podem ser tendenciosos. Os resultados baseados nesses diferentes métodos analíticos podem, portanto, ser inconsistentes, tornando a interpretação dos resultados do estudo clínico confusa. Em uma análise das diferentes metodologias feitas por um estudo em 2008 (143), conclui-se que LOCF consistentemente subestima as mudanças médias dentro do grupo de eficácia (benefício) e segurança (risco), enquanto MMRM e OC tendem a superestimar os resultados dentro do grupo.

A análise de subgrupos é um passo importante nas metanálises, pela limitação do número de estudos, tamanho das amostras e dados ausentes não foi possível realizar a mesma em relação a idade, sexo e tempo de diagnóstico do diabetes.

Um fator a ser considerado nesta análise é de perda de segmento nos estudos devido ao desenho. No estudo DAPA a previsão inicialmente de segmento era de dois anos, sendo que durante o perído, foram planejados dois anos adicionais. $O$ estudo EMPA foi idealizado para dois anos, com extensão de mais dois anos, sendo que alguns centros descontinuaram a segunda parte do estudo.

Outros fatores importantes a serem considerados são: os dados incompletos dos desfechos, relato seletivo e de desempenho (sub dose de sulfoniluréias como comparador).

Outros fatores não avaliados nesta revisão sistemática e que podem impactar a utilização clínica das gliflozinas e gliptinas são efeitos adversos, segurança 
cardiovascular e custo efetividade. A literatura indica um risco maior de infecção urinária e genital na classe das gliflozinas (78), estudos recentes tem indicado também um perfil cardiovascular benéfico para as mesmas $(142,144)$. O custo efetividade das classes ainda precisa ser estudado na população brasileira. 


\section{CONCLUSÕES}

Faltam estudos em longo prazo para avaliar a eficácia das gliptinas e gliflozinas após falência terapêutica de monoterapia da metformina em pacientes com DM2.

Foi identificado somente um estudo com segmento de quatro anos para gliptinas (saxagliptina), porém com dados críticos faltantes.

A eficácia do tratamento, definida pela porcentagem de pacientes com $\mathrm{HbA} 1 \mathrm{c}$ $\leq 7 \%$, é baixa em menos de um ano de segmento para as gliflozinas (empagliflozina e dapagliflozina) $<50 \%$. Em quatro anos de segmento, o perfil de eficácia da empagliflozina (23\%) foi melhor que para saxagliptina $(7 \%)$ e da dapagliflozina $(5 \%)$, porém com valores médios de $\mathrm{HbA} 1 \mathrm{c}$ não estatisticamente significantes. A qualidade da evidência é baixa.

A variação da perda de peso foi sustentada em quatro anos para as gliflozinas em relação às sulfoniluréias (DMP 1,1 a $2,6 \mathrm{Kg}$ ), sendo mais favorável a empagliflozina. A qualidade da evidência é baixa.

A frequência de pacientes com hipoglicemia foi significativamente melhor para as gliflozinas em relação as sulfoniluréias. Não houve diferenças dentro da classe das gliflozinas. A qualidade da evidência é moderada.

A heterogeneidade observada nas análises, advém do desenho dos estudos e impacta na validade externa dos achados. Desta forma, os resultados desta revisão sistemática e metanálise sugerem cautela nas generalizações dos resultados para outras populações.

Mais estudos são necessários para avaliar eficácia do controle glicêmico, variação de peso e frequência de hipoglicemia em longo prazo após introdução das gliflozinas e gliptinas na falência primária de metformina em pacientes com DM2. 


\section{ANEXOS}

Anexo A. Aprovação Comitê de Ética FMUSP

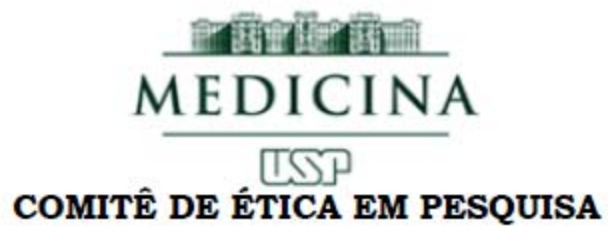

APROVAÇÃO

o Comitê de Ética em Pesquisa da Faculdade de Medicina da Universidade de São Paulo, em sessão de 15/07/2015, APROVOU o Protocolo de Pesquisa $n^{\circ}$ 293/15 intitulado: "ESTUDO DOS INIBIDORES DA DIPEPTIDIL PEPTIDASE-4 (IDPP-4) VERSUS INIBIDORES SELETIVOS DA SÓDIO-CO-TRANSPORTADOR DE GLICOSE 2 (SGLT2) APÓS FALÊNCIA DO TRATAMENTO COM METFORMINA EM PACIENTES COM DIABETES MELLITUS TIPO ^" apresentado pelo Departamento de CLÍNICA MÉDICA

Cabe ao pesquisador elaborar e apresentar ao CEPFMUSP, os relatórios parciais e final sobre a pesquisa (Resolução do Conselho Nacional de Saúde no 466/12, inciso IX.2, letra "c").

Pesquisador (a) Responsável: Fabiano Pinheiro da Silva Pesquisador (a) Executante: Renato Wilberto Zilli

CEP-FMUSP, 15 de Julho de 2015.

$$
\text { Rluamin. }
$$

Prof. Dr. Roger Chammas Coordenador Comitê de Ética em Pesquisa 


\section{Anexo B. Registro na base PROSPERO}

\section{PROSPERO International prospective register of systematic reviews}

\section{Review title and timescale}

1 Review title

Long-term efficacy of gliflozins versus gliptins in the treatment of type 2 diabetes mellitus after metformin failure as monotherapy: systematic review and network meta-analysis

2 Original language title

Eficácia em longo prazo das gliflozinas versus gliptinas no tratamento do diabetes mellitus tipo 2 após falência da metformina como monoterapia: revisão sistemática e metanálise em rede

3 Anticipated or actual start date

01 September 2015

4 Anticipated completion date

01 September 2017

5 Stage of review at time of this submission

Indicate the stage of progress of the review by ticking the relevant boxes. Reviews that have progressed beyond the point of completing data extraction at the time of initial registration are not eligible for inclusion in PROSPERO. This field should be updated when any amendments are made to a published record.

The review has not yet started $x$

Review stage

Preliminary searches

Piloting of the study selection process

Formal screening of search results against eligibility criteria

Data extraction

Risk of bias (quality) assessment

Data analysis

Provide any other relevant information about the stage of the review here.

Review team details

$6 \quad$ Named contact

Renato Zilli

$7 \quad$ Named contact email

rwzilli@gmail.com

$8 \quad$ Named contact address

Alameda Jau, 605 ap 151 São Paulo - SP Brazil Zip 01420-000

$9 \quad$ Named contact phone number

+5511996317432

10 Organisational affiliation of the review

University of Sao Paulo Medical School.

http://www.fm.usp.br/site/Home

11 Review team members and their organisational affiliations

Give the title, first name and last name of all members of the team working directly on the review. Give the organisational affiliations of each member of the review team.

$\begin{array}{lll}\text { Title } & \text { First name } & \text { Last name } \\ \mathrm{Dr} & \text { Renato } & \text { Zilli } \\ \mathrm{Dr} & \text { Fabiano } & \text { Pinheiro } \\ \mathrm{Dr} & \text { Renato } & \text { Baena } \\ \mathrm{Dr} & \text { Fernando } & \text { Zampieri }\end{array}$

12 Funding sources/sponsors

University of Sao Paulo Medical School

13 Conflicts of interest

None

14 Collaborators

Give the name, affiliation and role of any individuals or organisations who are working on the review but who are not listed as review team members.

Mrs Rosa $\quad$ Fischi

$\begin{array}{ll}\text { Started } & \text { Completed } \\ \text { Yes } & \text { Yes } \\ \text { Yes } & \text { No } \\ \text { No } & \text { No } \\ \text { No } & \text { No } \\ \text { No } & \text { No } \\ \text { No } & \text { No }\end{array}$

Affiliation

University of Sao Paulo Medical School

University of Sao Paulo Medical School

University of Sao Paulo Medical School

University of São Paulo Faculty of Medicine

Clinics Hospital

\section{Review methods}

15 Review question(s) 
Compare long-term efficacy (HbA1c, weight and hypoglycemia) of dipeptidyl peptidase-4 (DPP-4) inhibitors versus sodium-glucose co-transporter 2 (SGLT2) inhibitors in patients with type 2 diabetes inadequately controlled with metformin.

16 Searches

We will search the following electronic bibliographic databases: MEDLINE (PubMed), EMBASE, Web of Science the Cochrane Central Register of Controlled Trials (CENTRAL), Lilacs and Clinical Trials (http://www.clinicaltrials.gov).

We will search the 2015 conference proceedings of American Diabetes Association and European Association of the

Study of Diabetes (EASD).

The search strategy will include only terms relating to or describing the intervention. The terms will be combined with the Cochrane MEDLINE filter for controlled trials of interventions. The search strategy for MEDLINE is available in the published protocol. The search terms will be adapted for use with other bibliographic databases in combination with database-specific filters for controlled trials, where these are available.

There will be no language restrictions. Studies published will be consider until the date of search.

17 URL to search strategy

If you have one, give the link to your search strategy here. Alternatively you can e-mail this to PROSPERO and we will store and link to it.

I give permission for this file to be made publicly available Yes

18 Condition or domain being studied

Treatment options in type 2 diabetes, comparing of dipeptidyl peptidase-4 (DPP-4) inhibitors versus sodiumglucose co-transporter 2 (SGLT2) inhibitors

19 Participants/population

Inclusion: clinical trials that evaluated the efficacy of treatment of DPP-4 inhibitors or SGLT2 inhibitors in patients with type 2 diabetes inadequately controlled with metformin after 52 weeks of treatment or more. Exclusion: Non-RCT trials, RCT trials shorter than 52 weeks, animal studies

20 Intervention(s), exposure(s)

RCT's in type 2 diabetes patients inadequately controlled with metformin evaluating the efficacy of DPP-4 inhibitors or SGT2 inhibitors lasting more than 52 weeks.

21 Comparator(s)/control

These drugs class are relatively new. We decided to do a network analysis including other hypoglycemic agents as comparators.

22 Types of study to be included initially

We will include randomized trials to assess the efficacy of DPP-4 inhibitors and SGT2 inhibitors after metformin treatment failure in patients with type 2 diabetes.

23 Context Compare long-term efficacy (HbA1c, weight and hypoglycemia) of dipeptidyl peptidase-4 (DPP-4) inhibitors versus sodium-glucose co-transporter 2 (SGLT2) inhibitors in patients with type 2 diabetes inadequately controlled with metformin.

24 Primary outcome(s)

Primary outcome is mean change in $\mathrm{HbA} 1 \mathrm{c}$ after 52 weeks of treatment.

25 Secondary outcomes

Secondary outcomes are weight range and the occurrence of hypoglycemias.

26 Data extraction, (selection and coding)

Our draft eligibility criteria will be pilot-tested by the team. Subsequently, two reviewers will screen the literature search results in duplicate. Conflicts will be resolved by team discussion. We will follow a consistent process for screening full-text articles and abstracting data from included studies. We will abstract data on the following characteristics: studies (e.g., location of study conduct, study design), patients (e.g., mean age and standard deviation), and results (mean change from baseline $\mathrm{HbA} 1 \mathrm{c}$ and weight and at least one episode of hypoglycemia analyzed on an odds ratio scale)

27 Risk of bias (quality) assessment

The quality of eligible studies will be evaluated according to the Cochrane Collaboration's risk of bias tool for assessing risk of bias.

28 Strategy for data synthesis

Using the results of the systematic review to create a network of evidence, indirect comparisons will be made comparing the relative effects of treatments against the comparators.

29 Analysis of subgroups or subsets 'one planned.

Review general information 
30 Type of review

Intervention.

31 Language

English, Portuguese

32 Country

Brazil

33 Other registration details

Ethics Committees of University of Sao Paulo Medical School registration number - 293/15.

34 Reference and/or URL for published protocol

None.

I give permission for this file to be made publicly available

Yes

35 Dissemination plans

Give brief details of plans for communicating essential messages from the review to the appropriate audiences.

Do you intend to publish the review on completion?

Yes

36 Keywords

Type 2 diabetes, metformin, treatment outcome, HbA1c, DPP-4 inhibitors, SGT2 inhibitors

37 Details of any existing review of the same topic by the same authors None.

38 Current review status

Review status should be updated when the review is completed and when it is published.

39 Any additional information

Provide any further information the review team consider relevant to the registration of the review.

40 Details of final report/publication(s)

This field should be left empty until details of the completed review are available.

Give the full citation for the final report or publication of the systematic review.

Give the URL where available. 


\section{Anexo C. Busca nas bases de dados}

\section{Base Pubmed via Medline}

("Diabetes Mellitus, Type 2"[Mesh]) OR

(Diabetes mellitus, Type 2) OR

(NIDDM) OR

(Maturity-Onset Diabetes) OR

(Diabetes Mellitus, Noninsulin-Dependent) OR

(Diabetes Mellitus, Adult-Onset) OR

(Adult-Onset Diabetes Mellitus) OR

(Diabetes Mellitus, Adult Onset) OR

(Diabetes Mellitus, Ketosis-Resistant) OR

(Diabetes Mellitus, Ketosis Resistant) OR

(Ketosis-Resistant Diabetes Mellitus) OR

(Diabetes Mellitus, Maturity-Onset) OR

(Diabetes Mellitus, Maturity Onset) OR

(Diabetes Mellitus, Non Insulin Dependent) OR

(Diabetes Mellitus, Non-Insulin-Dependent) OR

(Non-Insulin-Dependent Diabetes Mellitus) OR

(Diabetes Mellitus, Noninsulin Dependent) OR

(Diabetes Mellitus, Slow-Onset) OR

(Diabetes Mellitus, Slow Onset) OR

(Slow-Onset Diabetes Mellitus) OR

(Diabetes Mellitus, Stable) OR

(Stable Diabetes Mellitus) OR

(Diabetes Mellitus, Type II) OR

(Maturity-Onset Diabetes Mellitus) OR

(Maturity Onset Diabetes Mellitus) OR

(MODY) OR

(Type 2 Diabetes Mellitus) OR

(Noninsulin-Dependent Diabetes Mellitus) OR

(type 2 diabetes)

\section{AND}

("Metformin"[Mesh]) OR

(Dimethylbiguanidine) OR

(Dimethylguanylguanidine) OR

(Glucophage)

\footnotetext{
AND

("Dipeptidyl-Peptidase IV Inhibitors"[Mesh]) OR (Dipeptidyl Peptidase IV Inhibitors) OR (Inhibitors, Dipeptidyl-Peptidase IV) OR (Dipeptidyl-Peptidase 4 Inhibitors) OR (Dipeptidyl Peptidase 4 Inhibitors) OR (Gliptins) OR
} 
("sitagliptin" [Supplementary Concept]) OR

(Sitagliptin) OR

(4-oxo-4-(3-(trifluoromethyl)-5,6-dihydro(1,2,4) triazolo(4,3-a)pyrazin-7(8H)-yl)-1-

(2,4,5-trifluorophenyl)butan-2-amine) OR

(sitagliptin phosphate anhydrous) OR

(sitagliptin phosphate) OR

(sitagliptin monophosphate monohydrate) OR

(sitagliptin phosphate monohydrate) OR

(Januvia) OR

(MK 0431) OR

(MK0431) OR

(MK-0431) OR

("saxagliptin" [Supplementary Concept]) OR

(saxagliptin) OR

(3-hydroxyadamantylglycine-4,5-methanoprolinenitrile hydrate) OR

(Onglyza) OR

(BMS 477118) OR

(BMS477118) OR

(BMS-477118) OR

("vildagliptin" [Supplementary Concept]) OR

(vildagliptin) OR

((2S)-(((3-hydroxyadamantan-1-yl)amino)acetyl)pyrrolidine-2-carbonitrile) OR

(Galvus) OR

(NVP-LAF237) OR

("Linagliptin" [Supplementary Concept]) OR

(linagliptin) OR

(Tradjenta) OR

(BI 1356) OR

(BI1356) OR

(BI-1356) OR

("alogliptin" [Supplementary Concept]) OR

(alogliptin) OR

(nesina) OR

(2-((6-((3R)-3-aminopiperidin-1-yl)-3-methyl-2,4-dioxo-3,4-dihydropyrimidin-1(2H)yl)methyl) benzonitrile) OR

(SYR 322) OR

(SYR322) OR

(SYR-322) OR

("anagliptin" [Supplementary Concept]) OR

(anagliptin) OR

(anagliptin hydrochloride) OR

(N-(2-((2-((2S)-2-cyanopyrrolidin-1-yl)-2-oxoethyl)amino)-2-methylpropyl)-2-methyl pyrazolo(1,5-a)pyrimidine-6-carboxamide hydrochloride) OR

(3-(4-(4-(3-methyl-1-phenyl-1H-pyrazol-5-yl)piperazin-1-yl)pyrrolidin-2-

ylcarbonyl)thiazolidine" [Supplementary Concept]) OR

(teneligliptin) OR

("LC15-0444" [Supplementary Concept]) OR

(gemigliptin) OR

("trelagliptin" [Supplementary Concept]) OR

(trelagliptin) OR 
(SYR-472) OR

(trelagliptin succinate) $O R$

("gosogliptin" [Supplementary Concept]) OR

(Gosogliptin) OR

(PF-00734200) OR

(PF-734,200) OR

(PF-734200) OR

(xagliptin) OR

(retagliptin) OR

(evogliptin) OR

("dutogliptin" [Supplementary Concept]) OR

(dutogliptin) OR

(PHX1149T) OR

("denagliptin" [Supplementary Concept]) OR

(denagliptin) OR

(4-fluoro-1-(4-fluoro-(4-fluorophenyl)phenylalanyl)-2-pyrrolidinecarbonitrile) OR

("Sodium-Glucose Transporter 2"[Mesh]) OR

(Sodium Glucose Transporter 2) OR

(SGLT2 Protein) OR

(sodium-glucose cotransporter 2 (SGLT-2) inhibitors) OR

(sodium-glucose cotransporter 2 inhibitor) OR

(sodium glucose cotransporter 2 inhibitor) OR

(inhibitor of sodium-glucose cotransporter 2) OR

(SGLT2 inhibitor) OR

("canagliflozin" [Supplementary Concept]) OR

(canagliflozin) OR

(Invokana) OR

(1-(glucopyranosyl)-4-methyl-3-(5-(4-fluorophenyl)-2-thienylmethyl)benzene) OR

("2-(3-(4-ethoxybenzyl)-4-chlorophenyl)-6-hydroxymethyltetrahydro-2H-pyran-3,4,5triol" [Supplementary Concept]) OR

(dapagliflozin) OR

((2S,3R,4R,5S,6R)-2-(4-chloro-3-(4-ethoxybenzyl)phenyl)-6-

(hydroxymethyl)tetrahydro-2H-pyran-3,4,5-triol) OR

(forxiga) OR

(BMS 512148) OR

(BMS512148) OR

(BMS-512148) OR

("empagliflozin" [Supplementary Concept]) OR

(empagliflozin) OR

(1-chloro-4-(glucopyranos-1-yl)-2-(4-(tetrahydrofuran-3-yloxy)benzyl)benzene) OR

(BI 10773) OR

(Bl10773) OR

(BI-10773) OR

(Jardiance) OR

("ipragliflozin" [Supplementary Concept]) OR

(ipragliflozin) OR

(Suglat) OR

("remogliflozin etabonate" [Supplementary Concept]) OR

(remogliflozin) OR

("sergliflozin" [Supplementary Concept]) OR 
(sergliflozin) OR

("6-((4-ethylphenyl)methyl)-3',4',5',6'-tetrahydro-6'-

(hydroxymethyl)spiro(isobenzofuran-1(3H),2'-(2H)pyran)-3',4',5'-triol" [Supplementary Concept]) OR

(CSG452) OR

(Apleway) OR

(Deberza) OR

(tofogliflozin) OR

(1,5-anhydro-1-(5-(4-ethoxybenzyl)-2-methoxy-4-methylphenyl)-1-thioglucitol

[Supplementary Concept]) OR

(luseogliflozin) OR

(Lusefi) OR

(TS 071) OR

(TS071 cpd) OR

(TS-071) OR

(bexagliflozin) OR

(ertugliflozin) OR

("3-(4-(4-(3-methyl-1-phenyl-1H-pyrazol-5-yl)piperazin-1-yl)pyrrolidin-2-

ylcarbonyl)thiazolidine" [Supplementary Concept]) OR

(teneligliptin) OR

("LC15-0444" [Supplementary Concept]) OR

(gemigliptin) OR

("carmegliptin" [Supplementary Concept]) OR

(carmegliptin) OR

("denagliptin" [Supplementary Concept]) OR

(denagliptin) OR

(evogliptin) OR

(DA-1229) OR

("isoleucyl-thiazolidide" [Supplementary Concept]) OR (isoleucine-thiazolidide) OR

(lle-thiazolidide) OR

(melogliptin) OR

("2-(2,5-difluorophenyl)-5-(2-(methylsulfonyl)-2,6-dihydropyrrolo(3,4-c)pyrazol-5(4H)yl)tetrahydro-2H-pyran-3-amine" [Supplementary Concept]) OR

(Omarigliptin) OR

(MK-3102) OR

(PSN-9301) OR

(PSN 9301) OR

("3-(4-(4-(3-methyl-1-phenyl-1H-pyrazol-5-yl)piperazin-1-yl)pyrrolidin-2-

ylcarbonyl)thiazolidine" [Supplementary Concept]) OR

(teneligliptin) OR

(atigliflozin) OR

(sotagliflozin)

AND

randomized controlled trial [pt] OR controlled clinical trial [pt] OR randomized [tiab] OR placebo [tiab] OR drug therapy [sh] OR randomly [tiab] OR trial [tiab] OR groups [tiab] NOT (animals[mh] NOT humans[Mesh]) 


\section{Busca na base Lilacs}

MH:("Diabetes Mellitus Tipo 2") OR

$\mathrm{MH}$ :("Diabetes Mellitus, Type 2") OR

TW:(Diabetes Mellitus de Início no Adulto) OR

TW:(Diabetes Mellitus Resistente a Cetose) OR

TW:(Diabetes Mellitus não Insulinodependente) OR

TW:(Diabetes Mellitus não Insulino-Dependente) OR

TW:(Diabetes Mellitus não Dependente de Insulina) OR

TW:(Diabetes Mellitus Estável) OR

TW:(DMNID) OR

TW:(Diabetes Mellitus de Início na Maturidade) OR

TW:(MODY) OR

TW:(Diabetes Mellitus de Início Gradativo) OR

$\mathrm{MH}: \mathrm{C} 18.452 .394 .750 .149^{*} \mathrm{OR}$

$\mathrm{MH}: \mathrm{C} 19.246 .300^{*}$

AND

$\mathrm{MH}:(" M e t f o r m i n a ~ ") ~ O R$

$\mathrm{MH}:($ "Metformin ") OR

TW:( Dimetil Guanil Guanidina) OR

$\mathrm{MH}$ : D02.078.370.141.450*

AND

MH:("Inibidores da Dipeptidil Peptidase IV") OR

$\mathrm{MH}$ :("Dipeptidyl-Peptidase IV Inhibitors") OR

$\mathrm{MH}$ :("Inhibidores de Dipeptidil-Peptidasa IV") OR

TW:(Gliptinas) OR

TW:(DPP4 inhibitor) OR

TW:(Inibidores da Dipeptidil Peptidase IV) OR

$\mathrm{MH}: \mathrm{D} 27.505 .519 .389 .745 .335^{*} \mathrm{OR}$

$\mathrm{MH}: \mathrm{D} 27.505 .696 .422 .500^{*} \mathrm{OR}$

TW:(sitagliptin) OR

TW:(saxagliptin) OR

TW:(vildagliptin) OR

TW:(linagliptin) OR

TW:(alogliptin) OR

TW:(anagliptin) OR

TW:(teneligliptin) OR

TW:(gemigliptin) OR

TW:(trelagliptin) OR

TW:(gosogliptin) OR

TW:(xagliptin) OR

TW:(retagliptin) OR

TW:(dutogliptin) OR

TW:(denagliptin) OR

TW:(bisegliptin) OR

TW:(carmegliptin) OR

TW:(evogliptin) OR 
TW:(melogliptin) OR

TW:(omarigliptin) OR

MH:("Transportador 2 de Glucose-Sódio") OR

MH:("Sodium-Glucose Transporter 2") OR

MH:("Transportador 2 de Sodio-Glucosa") OR

TW:(Transportador 2 de Sódio-Glucose) OR

TW:(Transportador de Glucose-Sódio, 2) OR

TW:(Transportador de Sódio-Glucose, 2) OR

TW:(SGLT2) OR

TW:(SGLT2 inhibitor) OR

$M H: D 12.776 .157 .530 .450 .625 .437 .750^{*}$ OR

$\mathrm{MH}: \mathrm{D} 12.776 .157 .530 .500 .750 .750^{*} \mathrm{OR}$

$M H: D 12.776 .543 .585 .450 .625 .562 .750^{*}$ OR

$\mathrm{MH}: \mathrm{D} 12.776 .543 .585 .500 .750 .750 * \mathrm{OR}$

TW:(canagliflozin) OR

TW:(dapagliflozin) OR

TW:(ipragliflozin) OR

TW:(empagliflozin) OR

TW:(remogliflozin) OR

TW:(sergliflozin) OR

TW:(tofogliflozin) OR

TW:(luseogliflozin) OR

TW:(bexagliflozin) OR

TW:(ertugliflozin) OR

TW:(atigliflozin) OR

TW:(sotagliflozin)

\section{Busca na base Central}

ID Search Hits

\#1 MeSH descriptor: [Diabetes Mellitus, Type 2] explode all trees 9267

\#2 MeSH descriptor: [Dipeptidyl-Peptidase IV Inhibitors] explode all trees 310

\#3 MeSH descriptor: [Sodium-Glucose Transporter 2] explode all trees 59

\#4 sitagliptin or saxagliptin or vildagliptin or linagliptin or alogliptin or anagliptin or teneligliptin or gemigliptin or trelagliptin or gosogliptin or xagliptin or retagliptin or dutogliptin or denagliptin or canagliflozin or dapagliflozin or ipragliflozin or empagliflozin or remogliflozin or sergliflozin or tofogliflozin or luseogliflozin or bexagliflozin or ertugliflozin or bisegliptin or carmegliptin or evogliptin or melogliptin or omarigliptin or atigliflozin or sotagliflozin $\quad 1429$
\#5 metformin 4102
\#6 \#4 or \#3 or \#2 1474
\#7 \#1 and \#5 and \#6 236 
Mesh descriptor para metformina retornou somente - 1651, optado pelo termo livre

\section{Busca na base Embase}

\#1

'non insulin dependent diabetes mellitus'/exp OR

'non insulin dependent diabetes mellitus'

\#2

'Diabetes mellitus, Type 2' OR

'NIDDM' OR

'Maturity-Onset Diabetes' OR

'Diabetes Mellitus, Noninsulin-Dependent' OR

'Diabetes Mellitus, Adult-Onset' OR

'Adult-Onset Diabetes Mellitus' OR

'Diabetes Mellitus, Adult Onset' OR

'Diabetes Mellitus, Ketosis-Resistant' OR

'Diabetes Mellitus, Ketosis Resistant' OR

'Ketosis-Resistant Diabetes Mellitus' OR

'Diabetes Mellitus, Maturity-Onset' OR

'Diabetes Mellitus, Maturity Onset' OR

'Diabetes Mellitus, Non Insulin Dependent' OR

'Diabetes Mellitus, Non-Insulin-Dependent' OR

'Non-Insulin-Dependent Diabetes Mellitus' OR

'Diabetes Mellitus, Noninsulin Dependent' OR

'Diabetes Mellitus, Slow-Onset' OR

'Diabetes Mellitus, Slow Onset' OR

'Slow-Onset Diabetes Mellitus' OR

'Diabetes Mellitus, Stable' OR

'Stable Diabetes Mellitus' OR

'Diabetes Mellitus, Type II' OR

'Maturity-Onset Diabetes Mellitus' OR

'Maturity Onset Diabetes Mellitus' OR

'MODY' OR

'Type 2 Diabetes Mellitus' OR

'Noninsulin-Dependent Diabetes Mellitus' OR

'type 2 diabetes'

\#2

'sodium glucose cotransporter 2 inhibitor'/exp OR

'sodium glucose cotransporter 2 inhibitor'

\#3

'dipeptidyl peptidase iv inhibitor'/exp OR

'dipeptidyl peptidase iv inhibitor'

\#4

'sitagliptin'/exp OR sitagliptin OR

'saxagliptin'/exp OR saxagliptin OR 


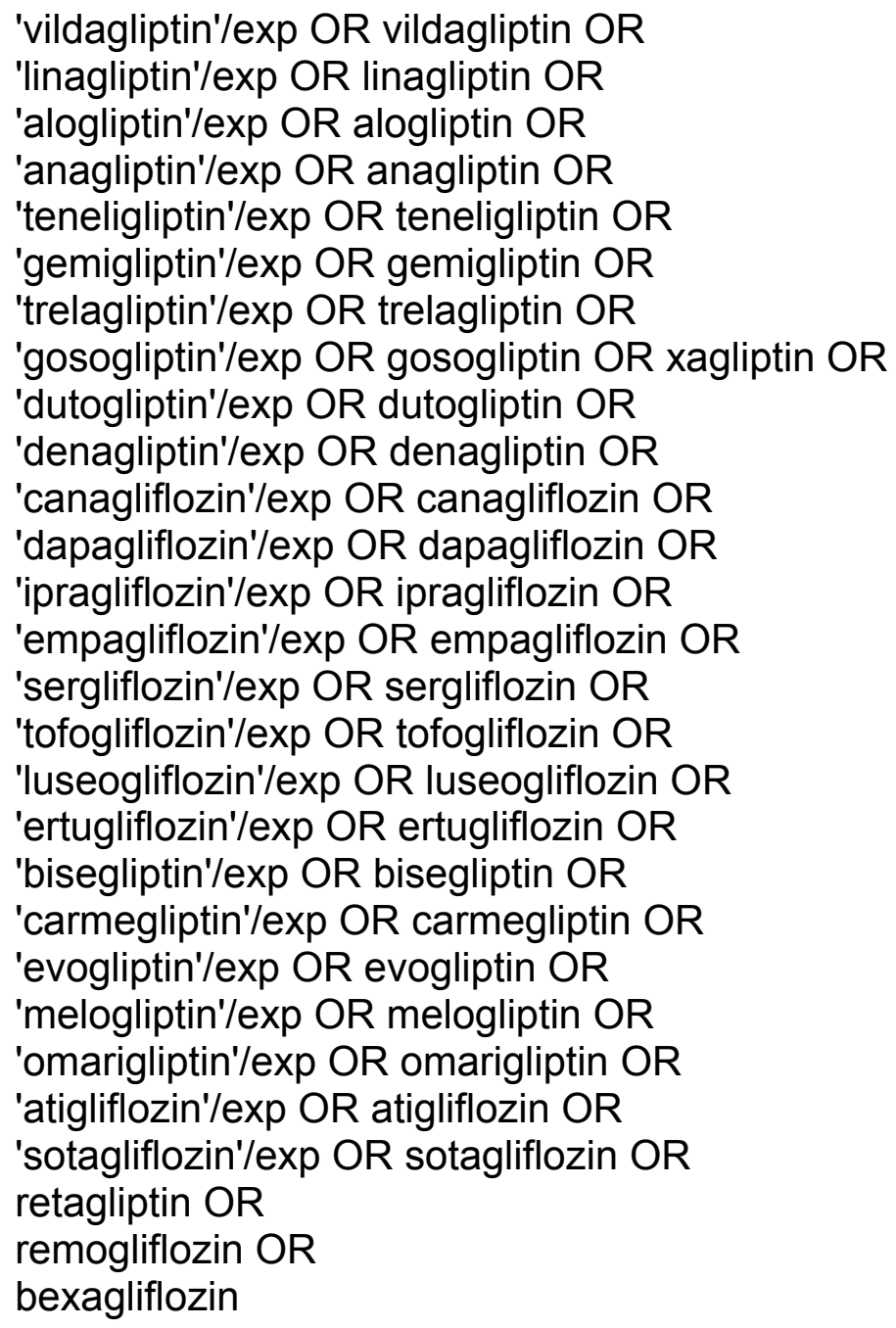

\# 5

'metformin'/exp OR 'metformin'

\#6

'crossover procedure':de OR 'double-blind procedure':de OR 'randomized controlled trial':de OR 'single-blind procedure':de OR random*:de,ab,ti OR factorial*:de, ab,ti OR crossover*:de,ab,ti OR (cross NEXT/1 over*):de,ab,ti OR placebo*:de, ab,ti OR (doubl* NEAR/1 blind*):de,ab,ti OR (singl* NEAR/1 blind*):de,ab,ti OR assign*:de,ab,ti OR allocat*:de,ab,ti OR volunteer*:de,ab,ti

\#7 \#1 OR \#2

\#8 \#2 OR \#3 OR \#4

\#9 \#5 AND \#6 AND \#7 AND \#8

\#10 'human'/de AND [embase]/lim NOT [medline]/lim

\#11 \#9 AND \#10 
Anexo D. Ficha padronizada de coleta para elegibilidade

Revisor:

\section{FORMULÁRIO DE AVALIAÇÃO DE ELEGIBILIDADE}
( ) Anna Maria Buehler
( ) Renato Baena
( ) Marcelo Silva
( ) Rodrigo Antonio Brandão
( ) Renato Zilli

Sobrenome do autor (Se a autoria pertencer a um grupo de Pesquisa, entrar com o primeiro nome significativo do grupo)

Nome do jornal (abreviação comumente utilizada, nome no PubMed ou nome completo do jornal)

\begin{tabular}{ll}
\hline Ano de Publicação & Volume \\
$\mathrm{N}^{\circ}$ da $1^{\circ}$ página do artigo &
\end{tabular}

\section{Critérios de Elegibilidade}

1. É um Ensaio Clínico Randomizado (ECR)?

$\square$ SIM $\square$ Não $\square$ Sem descrição/desconhecido

2. O estudo inclui portadores de diabetes mellitus tipo $\mathbf{2}$ em uso antes da randomização de metformina isoladamente?

$\square$ SIM $\square$ Não $\square$ Sem descrição/desconhecido

3. Algum dos braços da randomização é composto por iDDP-4 (inibidores da dipeptidil peptidase-4 I DDP-4 inhibitor ou inibidores seletivos da sódio-cotransportador de glicose 2 ( SGLT2 inhibitor) - sufixo gliptina ou glizofina)?

$\square$ SIM $\square$ Não $\square$ Sem descrição/desconhecido

4. Após a randomização, o segmento é igual ou maior a 24 semanas?

$\square$ SIM $\quad \square$ Não $\quad \square$ Sem descrição/desconhecido

Serão incluídos apenas estudos que são considerados elegíveis pelas instruções de elegibilidade do presente documento, isto é, caso todas as respostas para as questões a até 4 forem "sim". Caso as respostas para algumas das questões não esteja clara no texto, os autores serão contatados para esclarecimentos. 


\section{Anexo E. Ficha de extração de dados (em inglês)}

Data Extraction

\begin{tabular}{|l|l|l|}
\hline Study ID: & & Date form completed: \\
\hline First author: & Year of study: & Data extractor: \\
\hline \multicolumn{2}{|l|}{ Report ID's (type of article, abstract, site) } \\
\hline
\end{tabular}

Country of study:

Funding source of study:

Potential conflict of interest from funding? $\mathrm{Y} / \mathrm{N} / \mathrm{unclear}$

2. Study Eligibility

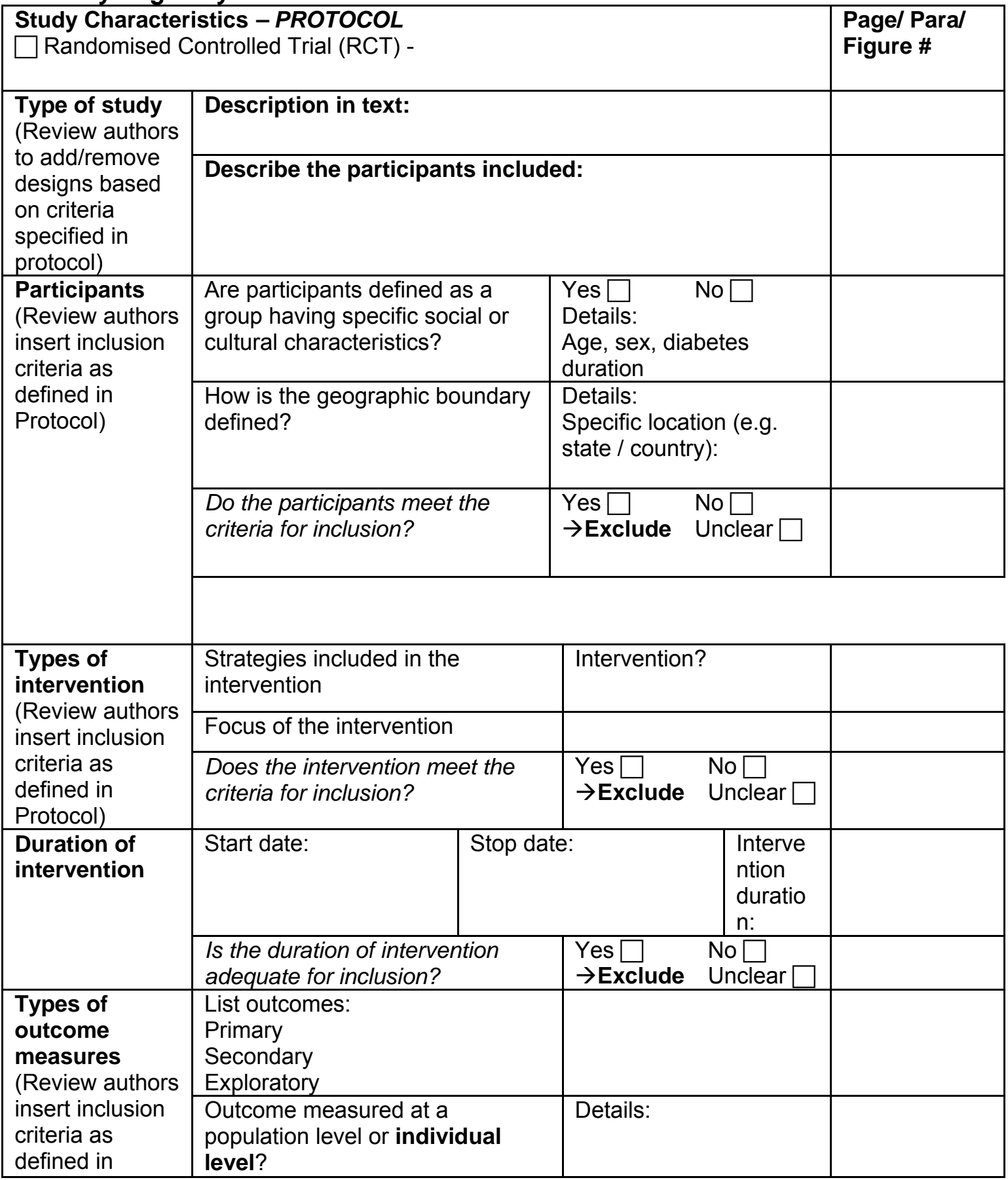




\begin{tabular}{|l|l|l|l|}
\hline Protocol) & $\begin{array}{l}\text { Do the outcome measures meet } \\
\text { the criteria for inclusion? }\end{array}$ & $\begin{array}{l}\text { Yes } \square \\
\rightarrow \text { Exclude Unclear } \square\end{array}$ & \\
\hline
\end{tabular}

3. Study details

\begin{tabular}{|l|l|l|}
\hline Study intention & Descriptions as stated in the report/paper & $\begin{array}{l}\text { Pagel Paral } \\
\text { Figure \# }\end{array}$ \\
\hline $\begin{array}{l}\text { Aim of } \\
\text { intervention }\end{array}$ & $\begin{array}{l}\text { What was the problem that this intervention was designed } \\
\text { to address? }\end{array}$ & \\
\hline Aim of study & $\begin{array}{l}\text { What was the study designed to assess? Are these } \\
\text { clearly stated? }\end{array}$ & $\begin{array}{l}\text { e.g. was study conducted in a particular setting that might } \\
\text { target/exclude specific populations? See also } \\
\text { Inclusion/exclusion criteria under Methods, below. }\end{array}$ \\
\hline $\begin{array}{l}\text { Equity pointer: } \\
\text { Social context of } \\
\text { the study }\end{array}$ & & \\
\hline $\begin{array}{l}\text { Total study } \\
\text { duration }\end{array}$ & & \\
\hline
\end{tabular}

\begin{tabular}{|l|l|l|}
\hline Methods & $\begin{array}{l}\text { Descriptions as stated } \\
\text { in the report/paper }\end{array}$ & $\begin{array}{l}\text { Pagel Paral } \\
\text { Figure \# }\end{array}$ \\
\hline $\begin{array}{l}\text { Method/s of recruitment of participants } \\
\text { (How were potential participants approached and } \\
\text { invited to participate? Where were participants } \\
\text { recruited from? Does this differ from the } \\
\text { intervention setting?) }\end{array}$ & & \\
\hline $\begin{array}{l}\text { Inclusion/exclusion criteria for participation in } \\
\text { study }\end{array}$ & & \\
\hline $\begin{array}{l}\text { Representativeness of sample: Are participants in } \\
\text { the study likely to be representative of the target } \\
\text { population? }\end{array}$ & & \\
\hline Total number of intervention groups & & \\
\hline $\begin{array}{l}\text { Assumed risk estimate } \\
\text { (e. baseline or population risk noted in } \\
\text { Background) }\end{array}$ & References: & \\
\hline $\begin{array}{l}\text { Sample size calculation: } \\
\text { What assumptions were made? } \\
\text { Were these assumptions appropriate? }\end{array}$ & (Yes/No/Unclear) & \\
\hline $\begin{array}{l}\text { What was the unit of randomisation? } \\
\text { Allocation by individuals or cluster/groups }\end{array}$ & & \\
\hline $\begin{array}{l}\text { What was the unit of analysis? } \\
\text { Is this the same as the unit of randomisation? }\end{array}$ & (Yes/No/Unclear) & \\
\hline $\begin{array}{l}\text { Statistical methods used and appropriateness of } \\
\text { these methods }\end{array}$ & $\begin{array}{l}\text { (Check with your } \\
\text { statistician if unsure about } \\
\text { appropriateness) }\end{array}$ & \\
\hline
\end{tabular}

\section{Results}

\begin{tabular}{|l|l|l|}
\hline $\begin{array}{l}\text { Participants } \\
\text { Include if relevant }\end{array}$ & $\begin{array}{l}\text { Include information for each group (i.e. } \\
\text { intervention and controls) under study }\end{array}$ & $\begin{array}{l}\text { Page/ Paral } \\
\text { Figure \# }\end{array}$ \\
\hline $\begin{array}{l}\text { What percentage of selected } \\
\text { individuals agreed to } \\
\text { participate? }\end{array}$ & & \\
\hline
\end{tabular}


- Total number randomised (or total pop. at start of study for NRCTs)

- Number allocated to each intervention group (no. of individuals)

- Where there any significant baseline imbalances?

- Number and reason for (and sociodemographic differences of) withdrawals and exclusions for each intervention group

- Were patients who entered the study adequately accounted for?

- What percentage of patients completed the study?

- What percentage of participants received the allocated intervention of interest?

- Is the analysis performed by intervention allocation status (intention to treat) rather than the actual intervention received? Have any attempts been made to impute missing data?

- Age (median, mean and range if possible)

- Sex

- Race/Ethnicity

- Diabetes duration

- Diagnostic criteria Metformin failure

- Co-morbidity

Subgroups

Sex

Time since diabetes diagnosis

Age (> 65 years old)

\section{Intervention Group 1}

(copy and paste table for each Intervention group)

\begin{tabular}{|l|l|}
\hline Group name: & (State brief name for this intervention
\end{tabular} group.)

Pagel Paral Figure \#

Details of intervention or control condition (Include if relevant in sufficient detail for replication)

- Setting eg multicentre, university teaching hospitals, rural, metropolitan, school, workplace, community, GP

clinic, etc. 


\begin{tabular}{|c|c|}
\hline $\begin{array}{l}\text { - Theoretical basis (include key } \\
\text { references) }\end{array}$ & \\
\hline $\begin{array}{l}\text { - Content (list the strategies } \\
\text { intended and delivered) }\end{array}$ & \\
\hline $\begin{array}{l}\text { Did the intervention include } \\
\text { strategies to address } \\
\text { diversity/disadvantage? }\end{array}$ & $\begin{array}{l}\text { Enter a description of any relevant } \\
\text { strategies }\end{array}$ \\
\hline $\begin{array}{l}\text { Delivery (eg. Stages } \\
\text { (sequential or simultaneous), } \\
\text { timing, frequency, duration, } \\
\text { intensity, fidelity - process } \\
\text { indicators) }\end{array}$ & \\
\hline $\begin{array}{l}\text { Providers (who, number, } \\
\text { education/training in } \\
\text { intervention delivery, ethnicity } \\
\text { etc. if potentially relevant to } \\
\text { acceptance and uptake by } \\
\text { participants }\end{array}$ & \\
\hline $\begin{array}{l}\text { Co-interventions } \\
\text { Diet and exercise? } \\
\text { Other medications? }\end{array}$ & \\
\hline Duration of intervention & \\
\hline Duration of follow-up & \\
\hline
\end{tabular}

\section{Outcomes A1c, weight, hypoglaecemia}

(This table is set up for 2 outcome measure to save spaces, copy and paste table as often as required)

\begin{tabular}{|l|l|l|l|l|l|l|}
\hline \multicolumn{1}{|c|}{ Question } & $\begin{array}{c}\text { Outcom } \\
\text { e 1 } \\
\text { A1c }\end{array}$ & $\begin{array}{c}\text { Pagel Paral } \\
\text { Figure \# }\end{array}$ & $\begin{array}{c}\text { Outcom } \\
\text { e 2 } \\
\text { weight }\end{array}$ & $\begin{array}{c}\text { Pagel } \\
\text { Paral } \\
\text { Figure \# }\end{array}$ & $\begin{array}{c}\text { Outcome 3 } \\
\text { Hypoglaecemi } \\
\text { a } \\
\text { Frequency }\end{array}$ & $\begin{array}{c}\text { Pagel } \\
\text { Paral } \\
\text { Figure \# }\end{array}$ \\
\hline $\begin{array}{l}\text { Is there an analytic } \\
\text { framework applied } \\
\text { (e.g. logic model, } \\
\text { conceptual } \\
\text { framework)? }\end{array}$ & & & & & & \\
\hline $\begin{array}{l}\text { Outcome definition } \\
\text { (with diagnostic } \\
\text { criteria if relevant) }\end{array}$ & & & & & & \\
\hline $\begin{array}{l}\text { Type of outcome: Is } \\
\text { this a modifiable } \\
\text { variable (Community } \\
\text { level, neighbourhood } \\
\text { level, individual level) } \\
\text { or desired health } \\
\text { outcome }\end{array}$ & & & & & & \\
\hline $\begin{array}{l}\text { Time points } \\
\text { measured }\end{array}$ & & & & & & \\
\hline Time points reported & & & & & & \\
\hline $\begin{array}{l}\text { Is there adequate } \\
\text { latency for the } \\
\text { outcome to be }\end{array}$ & & & & & & \\
\hline
\end{tabular}




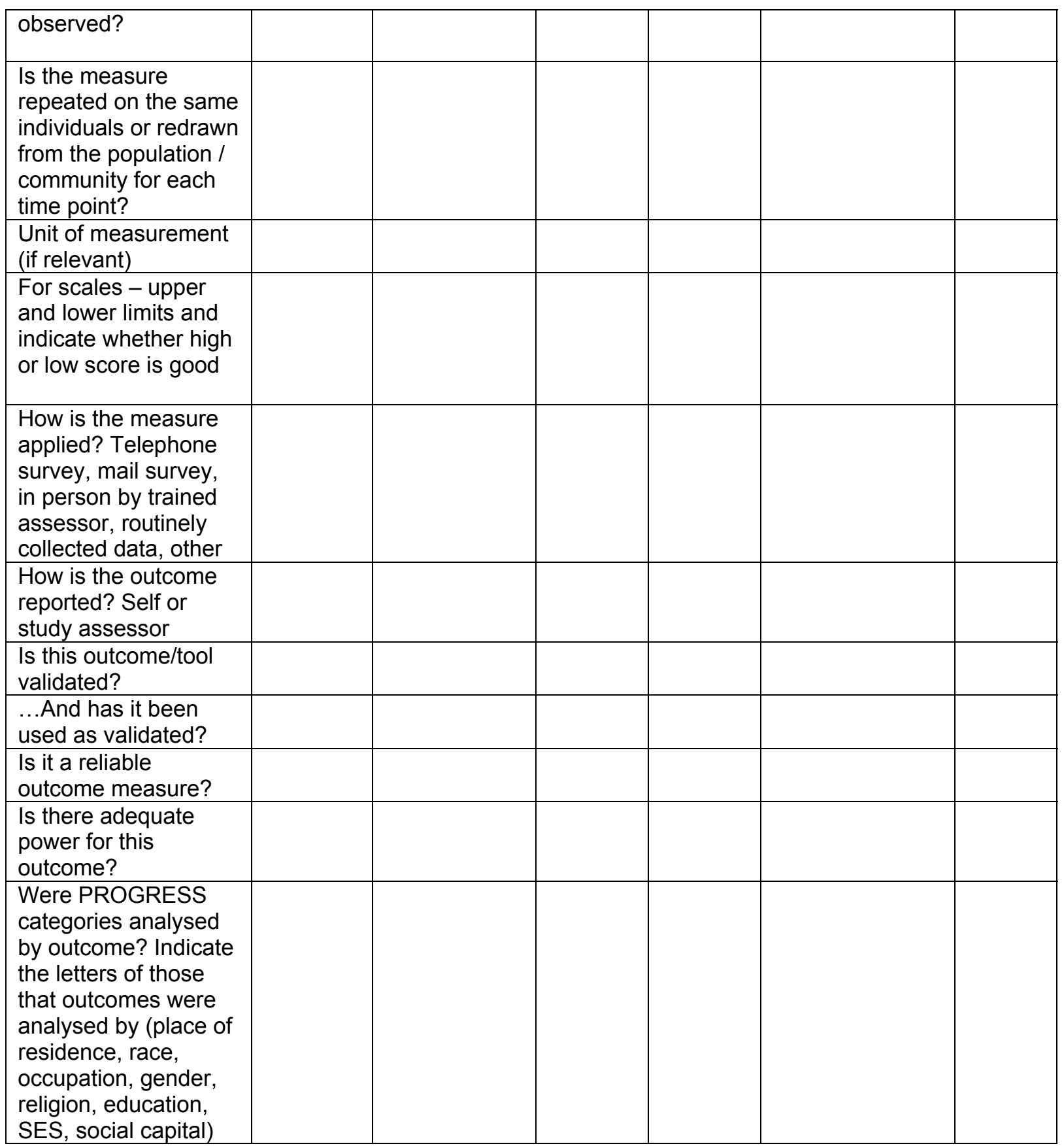




\section{Results}

Copy and paste the appropriate table for each outcome and subgroup at each timepoint, including baseline

For RCT/CCT

Dichotomous outcome

page/para/fig

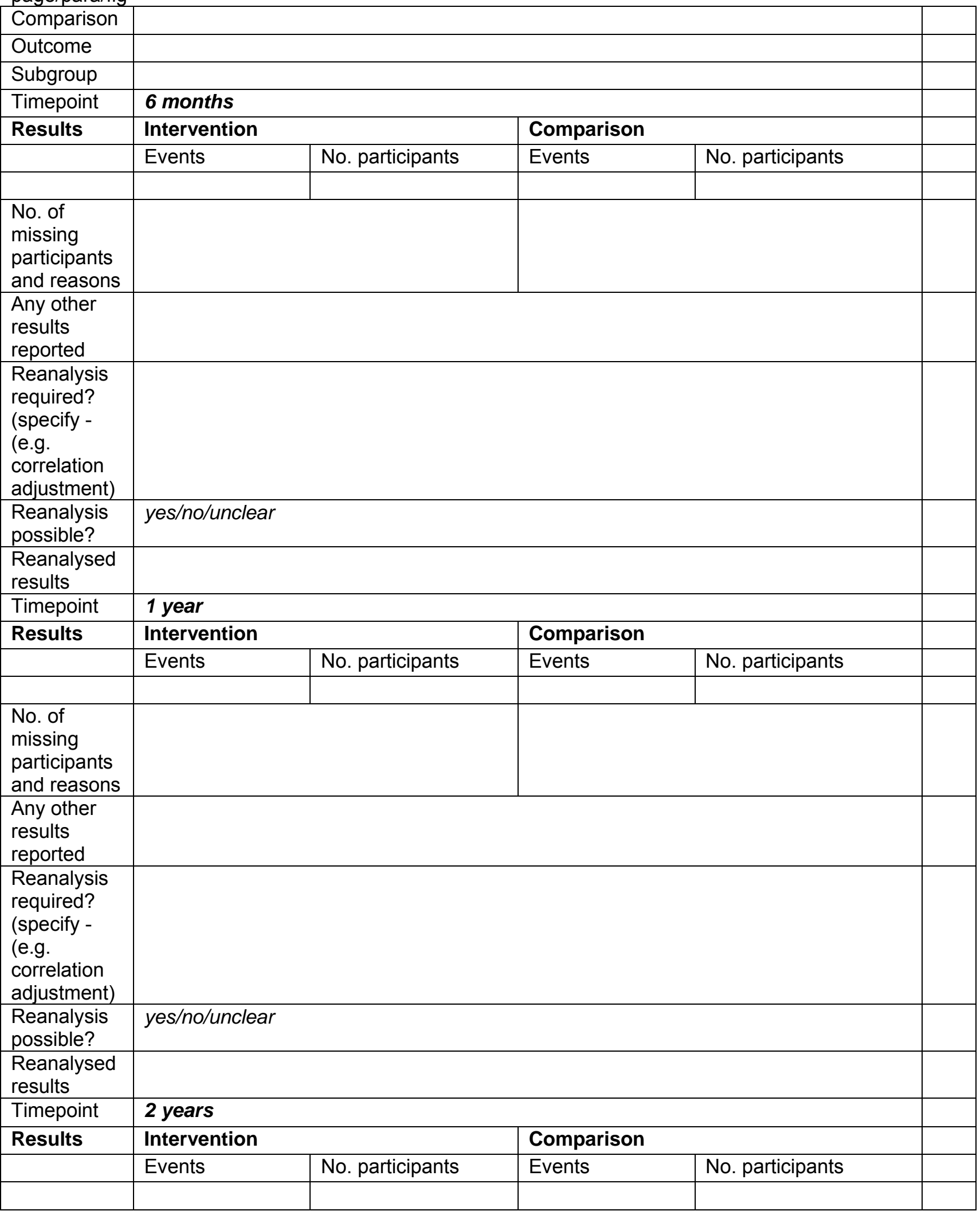




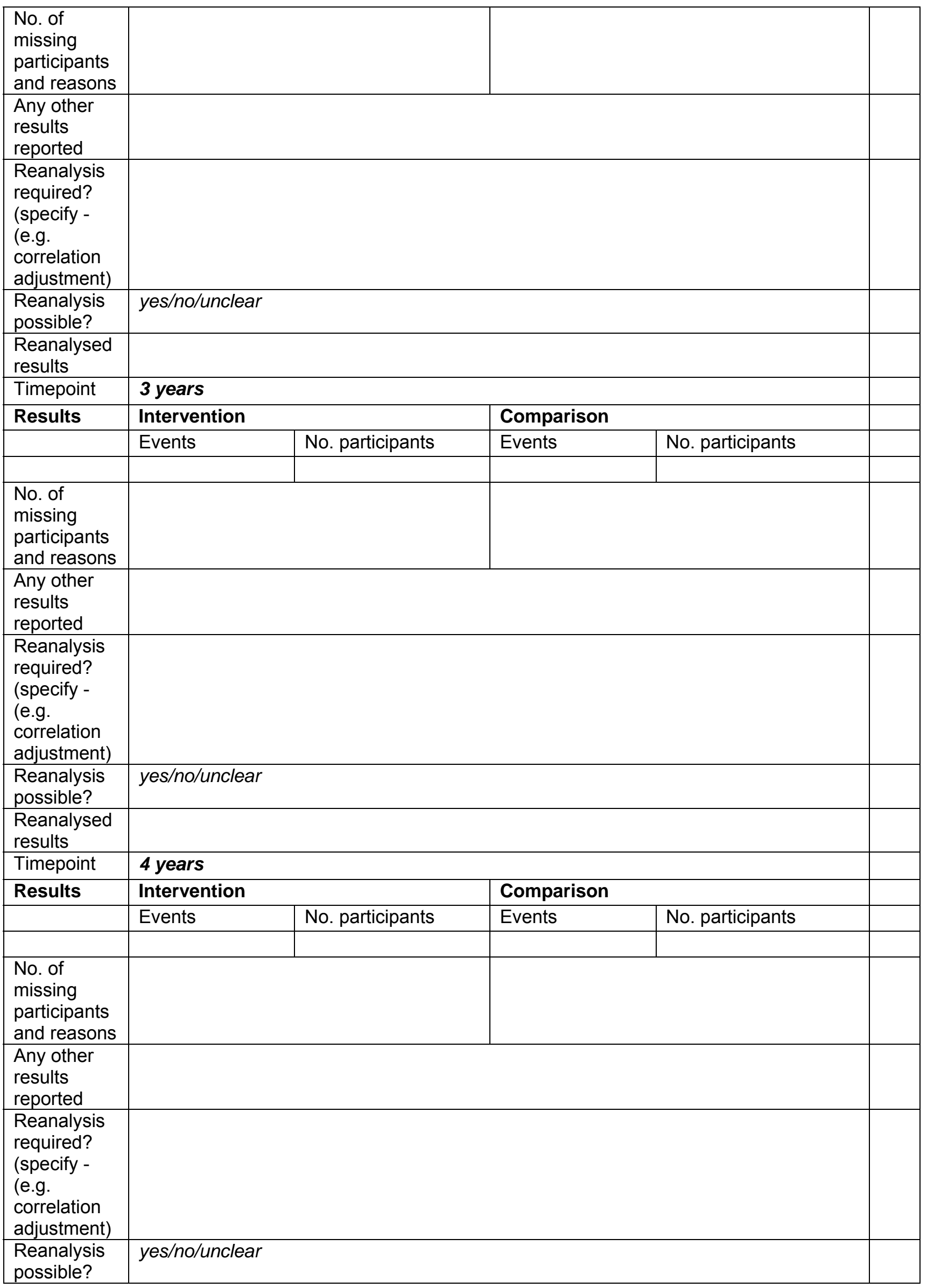


Reanalysed

results

For RCT/CCT

Continuous outcome

HbA1c

page/para/fig

Comparison

Outcome

Subgroup

Baseline

\begin{tabular}{|l|l|l|l|l|l|l|} 
& Mean & $\begin{array}{l}\text { SD (or } \\
\text { other } \\
\text { variance) }\end{array}$ & $\begin{array}{l}\text { No. } \\
\text { participants }\end{array}$ & Mean & $\begin{array}{l}\text { SD (or } \\
\text { other } \\
\text { variance) }\end{array}$ & $\begin{array}{l}\text { No. } \\
\text { participants }\end{array}$ \\
\cline { 2 - 7 } & \multicolumn{3}{|l|}{} & & & \\
\hline Timepoint & $\mathbf{6}$ months & & & & \\
\hline
\end{tabular}

Post-

intervention

or change

from

baseline?

Results

No. missing
participants

and

reasons

Any other

results

reported

Reanalysis

required?

(specify)

Reanalysis yes/no/unclear

possible?

Reanalysed

results

Timepoint

Post-

intervention

or change

from

baseline?

Results

No. missing

and

reasons

Any other

results

reported 


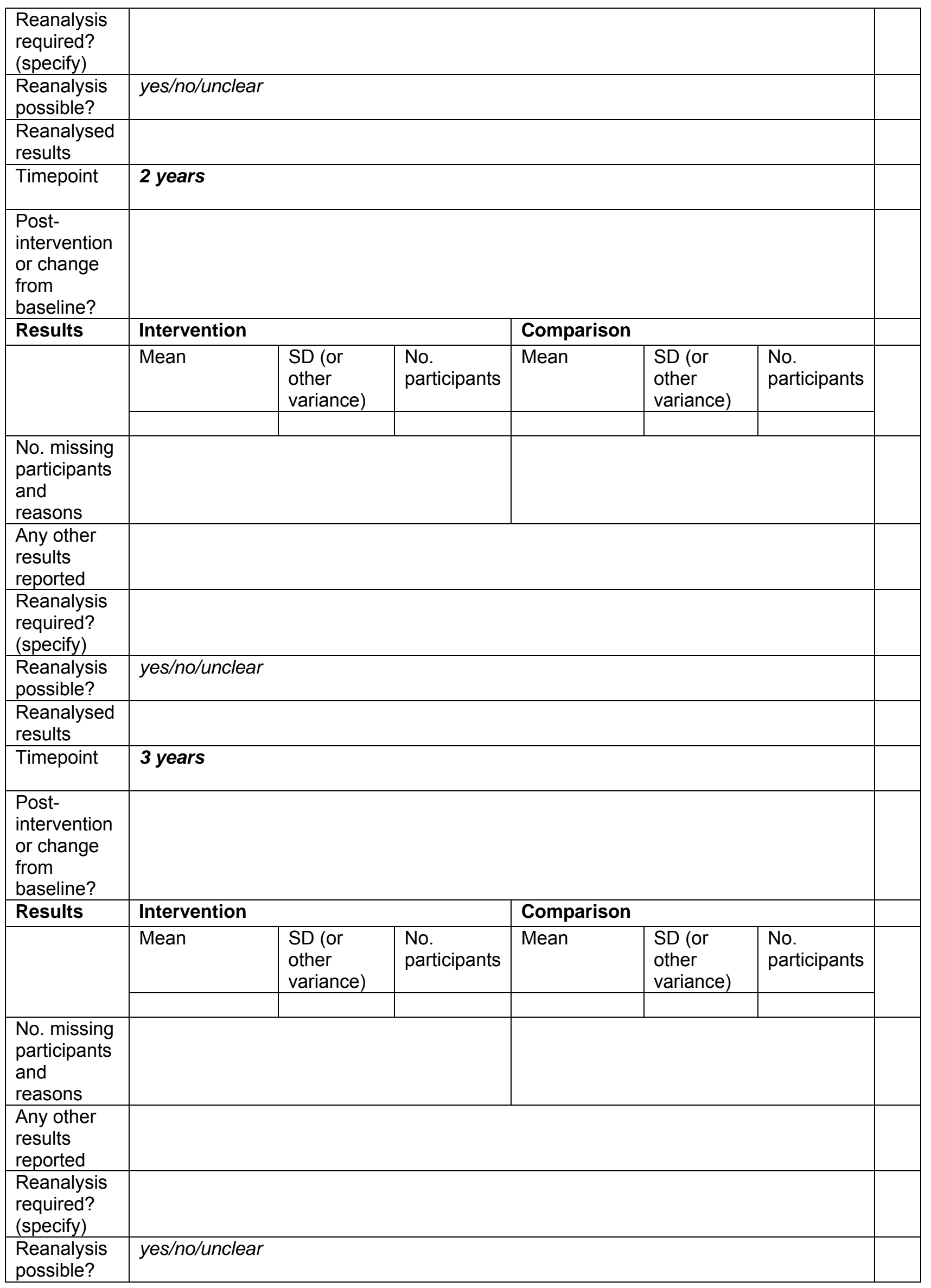




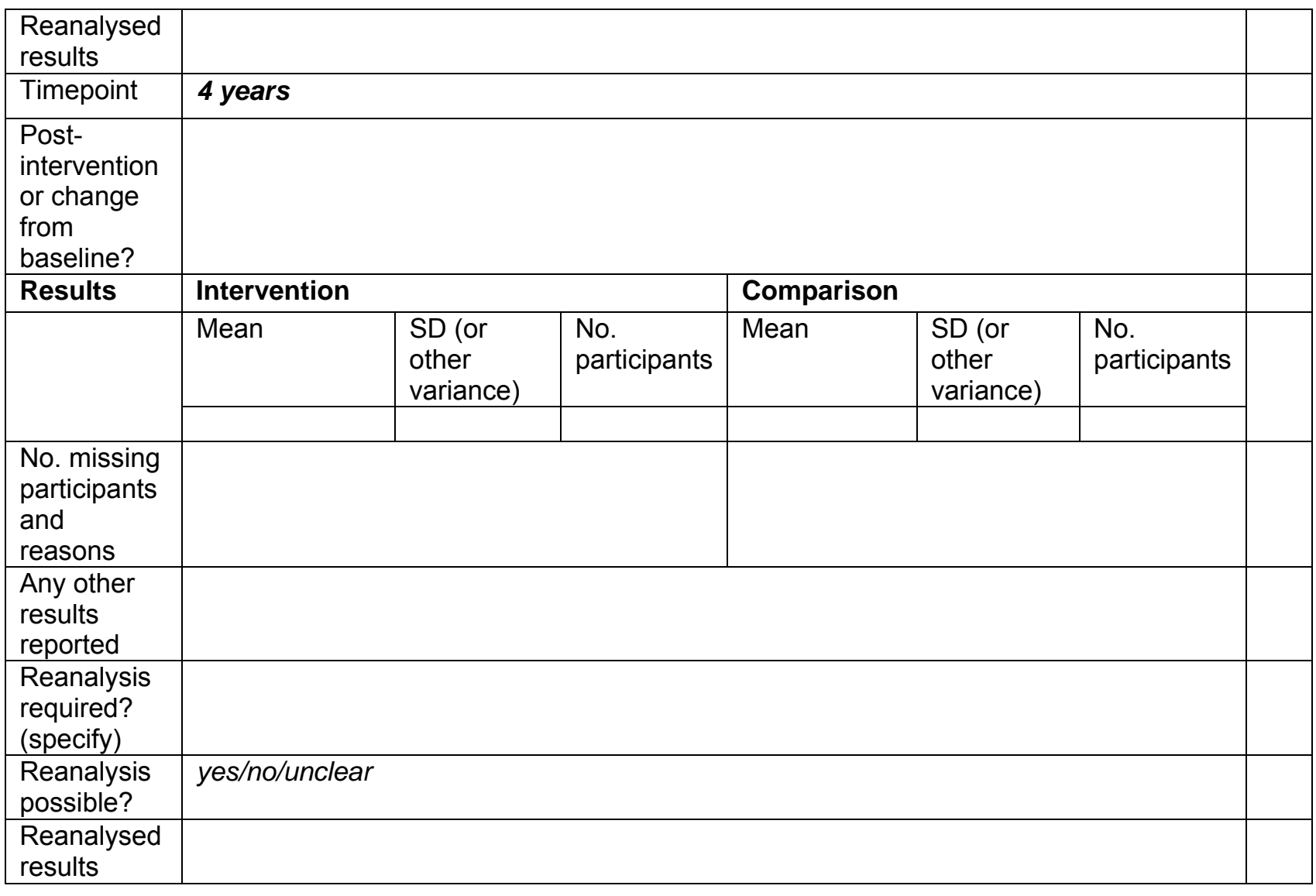

\section{Continuous outcome}

Weight

page/para/fig

\begin{tabular}{|c|c|c|c|c|c|c|}
\hline Comparison & & & & & & \\
\hline Outcome & & & & & & \\
\hline Subgroup & & & & & & \\
\hline Baseline & Interv & & & Comp & & \\
\hline & Mean & $\begin{array}{l}\text { SD (or } \\
\text { other } \\
\text { variance) }\end{array}$ & $\begin{array}{l}\text { No. } \\
\text { participants }\end{array}$ & Mean & $\begin{array}{l}\text { SD (or } \\
\text { other } \\
\text { variance) }\end{array}$ & $\begin{array}{l}\text { No. } \\
\text { participants }\end{array}$ \\
\hline Timepoint & $6 \mathrm{mon}$ & & & & & \\
\hline $\begin{array}{l}\text { Post- } \\
\text { intervention } \\
\text { or change } \\
\text { from } \\
\text { baseline? }\end{array}$ & & & & & & \\
\hline Results & Interv & & & Comp & & \\
\hline & Mean & $\begin{array}{l}\text { SD (or } \\
\text { other } \\
\text { variance) }\end{array}$ & $\begin{array}{l}\text { No. } \\
\text { participants }\end{array}$ & Mean & $\begin{array}{l}\text { SD (or } \\
\text { other } \\
\text { variance) }\end{array}$ & $\begin{array}{l}\text { No. } \\
\text { participants }\end{array}$ \\
\hline $\begin{array}{l}\text { No. missing } \\
\text { participants } \\
\text { and } \\
\text { reasons }\end{array}$ & & & & & & \\
\hline $\begin{array}{l}\text { Any other } \\
\text { results } \\
\text { renorted }\end{array}$ & & & & & & \\
\hline
\end{tabular}




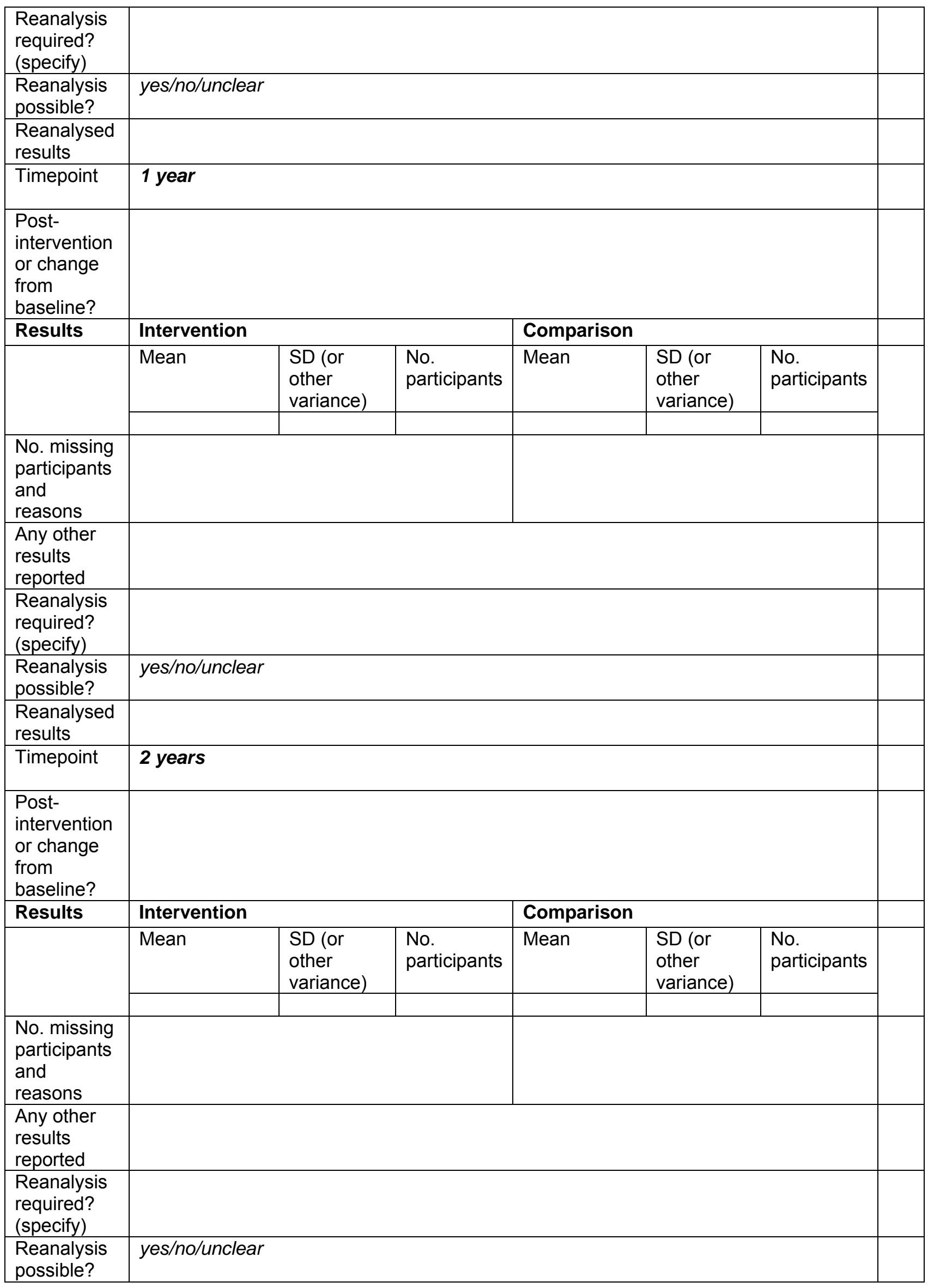




\begin{tabular}{|c|c|c|c|c|c|c|c|}
\hline \multirow{2}{*}{$\begin{array}{l}\text { Reanalysed } \\
\text { results } \\
\text { Timenoint }\end{array}$} & & & & & & & \\
\hline & \multicolumn{6}{|l|}{3 years } & \\
\hline \multicolumn{7}{|l|}{$\begin{array}{l}\text { Post- } \\
\text { intervention } \\
\text { or change } \\
\text { from } \\
\text { baseline? }\end{array}$} & \\
\hline Results & \multicolumn{3}{|l|}{ Intervention } & \multicolumn{3}{|c|}{ Comparison } & \\
\hline & Mean & $\begin{array}{l}\text { SD (or } \\
\text { other } \\
\text { variance) }\end{array}$ & $\begin{array}{l}\text { No. } \\
\text { participants }\end{array}$ & Mean & $\begin{array}{l}\text { SD (or } \\
\text { other } \\
\text { variance) }\end{array}$ & $\begin{array}{l}\text { No. } \\
\text { participants }\end{array}$ & \\
\hline \multicolumn{8}{|l|}{$\begin{array}{l}\text { No. missing } \\
\text { participants } \\
\text { and } \\
\text { reasons }\end{array}$} \\
\hline \multicolumn{8}{|l|}{$\begin{array}{l}\text { Any other } \\
\text { results } \\
\text { reported }\end{array}$} \\
\hline \multicolumn{7}{|l|}{$\begin{array}{l}\text { Reanalysis } \\
\text { required? } \\
\text { (specify) }\end{array}$} & \\
\hline $\begin{array}{l}\text { Reanalysis } \\
\text { possible? }\end{array}$ & \multicolumn{6}{|l|}{ yes/no/unclear } & \\
\hline \multicolumn{7}{|l|}{$\begin{array}{l}\text { Reanalysed } \\
\text { results }\end{array}$} & \\
\hline Timepoint & \multicolumn{6}{|l|}{4 years } & \\
\hline \multicolumn{8}{|l|}{$\begin{array}{l}\text { Post- } \\
\text { intervention } \\
\text { or change } \\
\text { from } \\
\text { baseline? }\end{array}$} \\
\hline Results & \multicolumn{3}{|l|}{ Intervention } & \multicolumn{3}{|c|}{ Comparison } & \\
\hline & Mean & $\begin{array}{l}\text { SD (or } \\
\text { other } \\
\text { variance) }\end{array}$ & $\begin{array}{l}\text { No. } \\
\text { participants }\end{array}$ & Mean & $\begin{array}{l}\text { SD (or } \\
\text { other } \\
\text { variance) }\end{array}$ & $\begin{array}{l}\text { No. } \\
\text { participants }\end{array}$ & \\
\hline $\begin{array}{l}\text { No. missing } \\
\text { participants } \\
\text { and } \\
\text { reasons }\end{array}$ & & & & & & & \\
\hline $\begin{array}{l}\text { Any other } \\
\text { results } \\
\text { reported }\end{array}$ & & & & & & & \\
\hline $\begin{array}{l}\text { Reanalysis } \\
\text { required? } \\
\text { (specify) }\end{array}$ & & & & & & & \\
\hline $\begin{array}{l}\text { Reanalysis } \\
\text { possible? }\end{array}$ & yes/no/unclear & & & & & & \\
\hline $\begin{array}{l}\text { Reanalysed } \\
\text { results }\end{array}$ & yes/no/unclear & & & & & & \\
\hline
\end{tabular}

\section{Other relevant information}




\begin{tabular}{|l|l|l|}
\hline $\begin{array}{l}\text { Were outcomes relating to harms/unintended } \\
\text { effects of the intervention described? Include } \\
\text { any data for these in the outcomes tables } \\
\text { above }\end{array}$ & & \\
\hline $\begin{array}{l}\text { Potential for author conflict ie. evidence that } \\
\text { author or data collectors would benefit if } \\
\text { results favoured the intervention under study } \\
\text { or the control }\end{array}$ & & \\
\hline Key conclusions of the study authors & & \\
\hline $\begin{array}{l}\text { Could the inclusion of this study potentially } \\
\text { bias the generalisability of the review? Equity } \\
\text { pointer: Remember to consider whether } \\
\text { disadvantaged populations may have been } \\
\text { excluded from the study. }\end{array}$ & & \\
\hline Additional notes by review authors & & \\
\hline
\end{tabular}

\section{Risk of bias assessment}

Please refer to Chapter 8 - Table 8.5.c: Criteria for judging risk of bias in the 'Risk of bias' assessment tool and to the Cochrane EPOC Group's guidance for assessing Risk of bias for studies with a separate control group (RCTs, CCTs, CBAs).

\begin{tabular}{|c|c|c|c|}
\hline Domain & $\begin{array}{l}\text { Review } \\
\text { authors' } \\
\text { judgement* }\end{array}$ & Description & $\begin{array}{l}\text { Pagel } \\
\text { Paral } \\
\text { Figure \# }\end{array}$ \\
\hline $\begin{array}{l}\text { Was the allocation } \\
\text { sequence } \\
\text { adequately } \\
\text { generated? }\end{array}$ & $\begin{array}{l}\text { Yes I No I } \\
\text { Unclear }\end{array}$ & $\begin{array}{l}\text { Describe the method used to generate the allocation sequence in } \\
\text { sufficient detail to allow an assessment of whether it should } \\
\text { produce comparable groups. }\end{array}$ & \\
\hline $\begin{array}{l}\text { Was allocation } \\
\text { adequately } \\
\text { concealed? }\end{array}$ & $\begin{array}{l}\text { Yes } I \text { No I } \\
\text { Unclear }\end{array}$ & $\begin{array}{l}\text { Describe the method used to conceal the allocation sequence in } \\
\text { sufficient detail to determine whether intervention allocations } \\
\text { could have been foreseen in advance of, or during, enrolment. }\end{array}$ & \\
\hline $\begin{array}{l}\text { Were baseline } \\
\text { outcome } \\
\text { measurements } \\
\text { similar? }\end{array}$ & $\begin{array}{l}\text { Yes/No/Uncl } \\
\text { ear }\end{array}$ & $\begin{array}{l}\text { Note whether baseline outcome measurements were reported } \\
\text { and whether there were any important differences between } \\
\text { groups. If there were important differences between groups, note } \\
\text { whether appropriate adjusted analysis was performed to account } \\
\text { for this. }\end{array}$ & \\
\hline $\begin{array}{l}\text { Were baseline } \\
\text { characteristics } \\
\text { similar? }\end{array}$ & $\begin{array}{l}\text { Yes/No/Uncl } \\
\text { ear }\end{array}$ & $\begin{array}{l}\text { Note whether baseline characteristics were reported and whether } \\
\text { there were any important differences between groups. }\end{array}$ & \\
\hline $\begin{array}{l}\text { Were incomplete } \\
\text { outcome data } \\
\text { adequately } \\
\text { addressed? } \\
\text { Assessments } \\
\text { should be made for } \\
\text { each main outcome } \\
\text { (or class of } \\
\text { outcomes). }\end{array}$ & $\begin{array}{l}\text { Yes I No I } \\
\text { Unclear }\end{array}$ & $\begin{array}{l}\text { Describe the completeness of outcome data for each main } \\
\text { outcome, including attrition and exclusions from the analysis. } \\
\text { State whether attrition and exclusions were reported, the } \\
\text { numbers in each intervention group (compared with total } \\
\text { randomized participants), reasons for attrition/exclusions where } \\
\text { reported, and any re-inclusions in analyses performed by the } \\
\text { review authors. }\end{array}$ & \\
\hline
\end{tabular}




\begin{tabular}{|c|c|c|}
\hline $\begin{array}{l}\text { Was knowledge of } \\
\text { the allocated } \\
\text { intervention } \\
\text { adequately } \\
\text { prevented during } \\
\text { the study? } \\
\text { Separate } \\
\text { assessments } \\
\text { should be made for } \\
\text { relevant groups of } \\
\text { people involved in } \\
\text { the study i.e } \\
\text { participants, } \\
\text { outcome } \\
\text { assessors, } \\
\text { investigators, data } \\
\text { assessors etc }\end{array}$ & $\begin{array}{l}\text { Yes I No I } \\
\text { Unclear }\end{array}$ & $\begin{array}{l}\text { Describe all measures used, if any, to blind study participants } \\
\text { and personnel from knowledge of which intervention a } \\
\text { participant received. Provide any information relating to whether } \\
\text { the intended blinding was effective, or whether blinding was } \\
\text { appropriate. } \\
\text { - Participants - yes, no, unclear [record supporting statement from } \\
\text { study]. } \\
\text { - Investigators - yes, no, unclear [record supporting statement } \\
\text { from study]. } \\
\text { - Outcomes assessors - yes, no, unclear [record supporting } \\
\text { statement from study]. } \\
\text { Data assessors - yes, no, unclear [record supporting statement from } \\
\text { study]. }\end{array}$ \\
\hline $\begin{array}{l}\text { Was the study } \\
\text { adequately } \\
\text { protected against } \\
\text { contamination? }\end{array}$ & $\begin{array}{l}\text { Yes/No/Uncl } \\
\text { ear }\end{array}$ & $\begin{array}{l}\text { State whether and how the possibility of contamination was } \\
\text { minimised by the study design/implementation. }\end{array}$ \\
\hline $\begin{array}{l}\text { Are reports of the } \\
\text { study free of } \\
\text { suggestion of } \\
\text { selective outcome } \\
\text { reporting? } \\
\text { Assessments } \\
\text { should be made for } \\
\text { each main outcome } \\
\text { (or class of } \\
\text { outcomes). }\end{array}$ & $\begin{array}{l}\text { Yes I No I } \\
\text { Unclear }\end{array}$ & $\begin{array}{l}\text { State how the possibility of selective outcome reporting was } \\
\text { examined by the review authors, and what was found. }\end{array}$ \\
\hline $\begin{array}{l}\text { Other sources of } \\
\text { bias } \\
\text { - }\end{array}$ & $\begin{array}{l}\text { Yes I No I } \\
\text { Unclear }\end{array}$ & $\begin{array}{l}\text { State any important concerns about bias not addressed in the } \\
\text { other domains in the tool. }\end{array}$ \\
\hline
\end{tabular}

* Note: For each section above 'Yes' indicates a 'low risk of bias'; 'No' indicates a 'high risk of bias'; 'Unclear' indicates an 'uncertain risk of bias'. When entering the data into RevMan, the options to choose from will be 'Low', 'High' and 'Unclear'

\section{Results}

Comparison: 
Outcome:

Subcategory:

\begin{tabular}{|c|c|l|c|}
\hline \multicolumn{2}{|l|}{ Treatment group: } & \multicolumn{1}{l|}{ Control group: } \\
\hline Observed $(\mathrm{n})$ & total $(\mathrm{N})$ & observed $(\mathrm{n})$ & total $(\mathrm{N})$ \\
\hline & & & \\
\hline
\end{tabular}

\begin{tabular}{|l|l|l|}
\hline & Treatment group: & Control group: \\
\hline Total randomised & & \\
\hline excluded* & & \\
\hline Observed & & \\
\hline lost to follow up* & & \\
\hline
\end{tabular}

*Reasons for loss/exclusion:

Subcategory:

\begin{tabular}{|c|c|c|c|}
\hline \multicolumn{2}{|l|}{ Treatment group: } & \multicolumn{1}{l|}{ Control group: } \\
\hline Observed $(\mathrm{n})$ & total $(\mathrm{N})$ & observed $(\mathrm{n})$ & total $(\mathrm{N})$ \\
\hline & & & \\
\hline
\end{tabular}

\begin{tabular}{|l|l|l|}
\hline & Treatment group: & Control group: \\
\hline Total randomised & & \\
\hline excluded* & & \\
\hline Observed & & \\
\hline lost to follow up* & & \\
\hline
\end{tabular}

*Reasons for loss/exclusion 


\section{Anexo F. Razão das exclusões}

Legenda:

1- Não é um estudo randomizado controlado

2- Não tem monoterapia prévia com metformina

3- Estudos com duração menor que dois anos ou subanálise destes.

\begin{tabular}{|c|c|c|c|}
\hline Titulo & Autores & Revista & Motivo \\
\hline $\begin{array}{l}\text { 2-year efficacy and safety of linagliptin } \\
\text { compared with glimepiride in patients } \\
\text { with type } 2 \text { diabetes inadequately } \\
\text { controlled on metformin: a randomised, } \\
\text { double-blind, non-inferiority trial. }\end{array}$ & $\begin{array}{l}\text { Gallwitz, Baptist; } \\
\text { Rosenstock, Julio; } \\
\text { Rauch, Thomas; } \\
\text { Bhattacharya, Sudipta; } \\
\text { Patel, Sanjay; von } \\
\text { Eynatten, Maximilian; } \\
\text { Dugi, Klaus A; Woerle, } \\
\text { Hans-Juergen }\end{array}$ & Lancet & 3 \\
\hline $\begin{array}{l}\text { Achieving reductions in HbA1c â\%o¥1\% } \\
\text { without hypoglycemia with saxagliptin } \\
\text { combination therapy: Post HOC } \\
\text { analysis of } 4 \text { randomized controlled } \\
\text { studies in patients with type } 2 \text { diabetes }\end{array}$ & $\begin{array}{l}\text { Karyekar, C.; } \\
\text { Frederich, R.; } \\
\text { Donovan, M.; } \\
\text { Ravichandran, S. }\end{array}$ & Diabetes & 3 \\
\hline $\begin{array}{l}\text { Achieving the composite end point of } \\
\mathrm{HbA} 1 \mathrm{c}<7 \% \text {, no hypos, and no weight } \\
\text { gain: Comparison between vildagliptin } \\
\text { and glimepiride after } 2 \text { years of } \\
\text { treatment }\end{array}$ & $\begin{array}{l}\text { Bader, G.; Ferrannini, } \\
\text { E.; Bo, A.; Fonseca, } \\
\text { V.; Zinman, B.; } \\
\text { Matthews, D. }\end{array}$ & Diabetologia & 3 \\
\hline $\begin{array}{l}\text { Achieving the composite end point of } \\
\mathrm{HbA} 1 \mathrm{c}<7 \% \text {, no hypos, and no weight } \\
\text { gain: Comparison between vildagliptin } \\
\text { and glimepiride after } 2 \text { years of } \\
\text { treatment }\end{array}$ & $\begin{array}{l}\text { Ferrannini, E.; Bader, } \\
\text { G.; Ahren, B.; } \\
\text { Fonseca, V.; Zinman, } \\
\text { B.; Matthews, D. }\end{array}$ & Diabetes & 3 \\
\hline $\begin{array}{l}\text { Addition of sitagliptin to ongoing } \\
\text { metformin monotherapy improves } \\
\text { glycemic control in Japanese patients } \\
\text { with type } 2 \text { diabetes over } 52 \text { weeks. }\end{array}$ & $\begin{array}{l}\text { Kadowaki,T.; } \\
\text { Tajima,N.; } \\
\text { Odawara,M.; Nishii,M.; } \\
\text { Taniguchi,T.; } \\
\text { Ferreira,J. C. A. }\end{array}$ & $\begin{array}{l}\text { Journal of Diabetes } \\
\text { Investigation }\end{array}$ & 1 \\
\hline $\begin{array}{l}\text { After one year of treatment, liraglutide } \\
\text { more successfully reduces } \mathrm{HbA} 1 \mathrm{c} \text { than } \\
\text { sitaglitpin across a broad range of }\end{array}$ & $\begin{array}{l}\text { Davies, M.; Pratley, R.; } \\
\text { Montanya Mias, E.; Xu, } \\
\text { Y.; Hartvig, H.; Sesti, }\end{array}$ & Diabetologia & 3 \\
\hline
\end{tabular}




\begin{tabular}{|c|c|c|c|}
\hline $\begin{array}{l}\text { baseline HbA1c values, when both are } \\
\text { combined with metformin }\end{array}$ & G. & & \\
\hline $\begin{array}{l}\text { Alogliptin superior to glipizide in } \\
\text { composites of a1c reduction, no } \\
\text { hypoglycemia, and no weight gain in } \\
\text { patients with T2DM }\end{array}$ & $\begin{array}{l}\text { Camisasca, R.; Bron, } \\
\text { M.; Fleck, P.; Wilson, } \\
\text { C.; Del Prato, S. }\end{array}$ & Diabetes & 3 \\
\hline $\begin{array}{l}\text { Anagliptin and sitagliptin as add-ons to } \\
\text { metformin for patients with type } 2 \\
\text { diabetes: A 24-week, multicentre, } \\
\text { randomized, double-blind, active- } \\
\text { controlled, phase III clinical trial with a } \\
\text { 28-week extension }\end{array}$ & $\begin{array}{l}\text { Jin, S. M.; Park, S. W.; } \\
\text { Yoon, K. H.; Min, K. } \\
\text { W.; Song, K. H.; Park, } \\
\text { K. S.; Park, J. Y.; Park, } \\
\text { I. B.; Chung, C. H.; } \\
\text { Baik, S. H.; Choi, S. } \\
\text { H.; Lee, H. W.; Lee, I. } \\
\text { K.; Kim, D. M.; Lee, M. } \\
\text { K. }\end{array}$ & $\begin{array}{l}\text { Diabetes, Obesity } \\
\text { and Metabolism }\end{array}$ & 3 \\
\hline $\begin{array}{l}\text { Association of patient-reported } \\
\text { hypoglycemia with health-related quality } \\
\text { of life (HRQL) in type } 2 \text { diabetes (T2D) } \\
\text { patients: Results from a clinical trial }\end{array}$ & $\begin{array}{l}\text { Gruenberger, J. B.; } \\
\text { Gilet, H.; EstÃ "̈ve, L.; } \\
\text { Viala-Danten, M. }\end{array}$ & Diabetes & 3 \\
\hline $\begin{array}{l}\text { Canagliflozin (CANA) compared with } \\
\text { sitagliptin (SITA) in subjects with type } 2 \\
\text { diabetes mellitus (T2DM) on metformin } \\
\text { (MET) over } 52 \text { weeks }\end{array}$ & $\begin{array}{l}\text { Lavalle GonzÃ ilez, F. } \\
\text { J.; Januszewicz, A.; } \\
\text { Davidson, J.; Qiu, R.; } \\
\text { Tong, C.; Meininger, } \\
\text { G. }\end{array}$ & Diabetes & 3 \\
\hline $\begin{array}{l}\text { Canagliflozin demonstrates durable } \\
\text { glycaemic improvements over } 104 \\
\text { weeks compared with glimepiride in } \\
\text { subjects with type } 2 \text { diabetes mellitus } \\
\text { on metformin }\end{array}$ & $\begin{array}{l}\text { Langslet, G.; Cefalu, } \\
\text { W. T.; Leiter, L. A.; } \\
\text { Yoon, K. H.; Arias, P.; } \\
\text { Xie, J.; Balis, D.; } \\
\text { Millington, D.; } \\
\text { Vercruysse, F.; } \\
\text { Canovatchel, W.; } \\
\text { Meininger, G. }\end{array}$ & Diabetologia & 3 \\
\hline $\begin{array}{l}\text { Canagliflozin demonstrates durable } \\
\text { glycemic improvements over } 104 \text { weeks } \\
\text { compared with glimepiride in subjects } \\
\text { with type } 2 \text { diabetes mellitus on } \\
\text { metformin }\end{array}$ & $\begin{array}{l}\text { Leiter, L. A.; Langslet, } \\
\text { G.; Cefalu, W. T.; Ho } \\
\text { Yoon, K.; Arias, P.; } \\
\text { Xie, J.; Balis, D.; } \\
\text { Millington, D.; } \\
\text { Vercruysse, F.; } \\
\text { Canovatchel, W.; } \\
\text { Meininger, G. }\end{array}$ & $\begin{array}{l}\text { Canadian Journal of } \\
\text { Diabetes }\end{array}$ & 3 \\
\hline
\end{tabular}




\begin{tabular}{|c|c|c|c|}
\hline $\begin{array}{l}\text { Canagliflozin is superior to sitagliptin in } \\
\text { reducing both } \mathrm{HbA} 1 \mathrm{c} \text { and body weight } \\
\text { in patients with type } 2 \text { diabetes mellitus }\end{array}$ & $\begin{array}{l}\text { Schernthaner, G.; } \\
\text { Hieronymus, L.; Jodon, } \\
\text { H.; Vijapurkar, U.; } \\
\text { Meininger, G.; } \\
\text { Canovatchel, W. }\end{array}$ & Diabetologia & 3 \\
\hline $\begin{array}{l}\text { Canagliflozin reduces both } \mathrm{HbA} 1 \mathrm{c} \text { and } \\
\text { body weight in patients with type } 2 \\
\text { diabetes mellitus on background } \\
\text { metformin }\end{array}$ & $\begin{array}{l}\text { Langslet, G.; } \\
\text { Davidson, J. A.; } \\
\text { Valentine, V.; } \\
\text { Vijapurkar, U.; } \\
\text { Canovatchel, W.; } \\
\text { Meininger, G. }\end{array}$ & Diabetologia & 3 \\
\hline $\begin{array}{l}\text { Canagliflozin, a sodium glucose co- } \\
\text { transporter } 2 \text { inhibitor, reduces body } \\
\text { weight mainly through loss of fat mass } \\
\text { in subjects with type } 2 \text { diabetes }\end{array}$ & $\begin{array}{l}\text { Toubro, S.; Cefalu, W. } \\
\text { T.; Xie, J.; Sullivan, D.; } \\
\text { Usiskin, K.; } \\
\text { Canovatchel, W.; } \\
\text { Meininger, G. }\end{array}$ & Diabetologia & 3 \\
\hline $\begin{array}{l}\text { Changes in prandial glucagon levels } \\
\text { after a 2-year treatment with vildagliptin } \\
\text { or glimepiride in patients with type } 2 \\
\text { diabetes inadequately controlled with } \\
\text { metformin monotherapy }\end{array}$ & $\begin{array}{l}\text { AhrÃ@n, B.; Foley, J. } \\
\text { E.; Ferrannini, E.; } \\
\text { Matthews, D. R.; } \\
\text { Zinman, B.; Dejager, } \\
\text { S.; Fonseca, V. A. }\end{array}$ & Diabetes care & 3 \\
\hline $\begin{array}{l}\text { Clinical experience with vildagliptin in } \\
\text { the management of type } 2 \text { diabetes in a } \\
\text { patient population }>/=75 \text { years: a } \\
\text { pooled analysis from a database of } \\
\text { clinical trials }\end{array}$ & $\begin{array}{l}\text { Schweizer, A.; } \\
\text { Dejager, S.; Foley, J. } \\
\text { E.; Shao, Q.; Kothny, } \\
\text { W. }\end{array}$ & $\begin{array}{l}\text { Diabetes, Obesity } \\
\text { and Metabolism }\end{array}$ & 3 \\
\hline $\begin{array}{l}\text { Combination of empagliflozin and } \\
\text { linagliptin as second-line therapy in } \\
\text { subjects with type } 2 \text { diabetes } \\
\text { inadequately controlled on metformin. }\end{array}$ & $\begin{array}{l}\text { DeFronzo RA.; Lewin } \\
\text { A.; Patel S.; Liu D.; } \\
\text { Kaste R.; Woerle HJ.; } \\
\text { Broedl UC. }\end{array}$ & Diabetes care & 3 \\
\hline $\begin{array}{l}\text { Comparative study of sitagliptin with } \\
\text { pioglitazone for strict glycemic control: } \\
\text { The compass study }\end{array}$ & $\begin{array}{l}\text { Takihata, M.; } \\
\text { Nakamura, A.; Tajima, } \\
\text { K.; Inazumi, T.; } \\
\text { Komatsu, Y.; Tamura, } \\
\text { H.; Yamazaki, S.; } \\
\text { Kondou, Y.; Yamada, } \\
\text { M.; Kimura, M.; } \\
\text { Terauchi, Y. }\end{array}$ & Diabetes & 3 \\
\hline $\begin{array}{l}\text { A comparison of efficacy and safety of } \\
\text { vildagliptin and gliclazide in combination }\end{array}$ & Filozof, C; Gautier, J-F & Diabetic medicine & 3 \\
\hline
\end{tabular}




\begin{tabular}{|c|c|c|c|}
\hline $\begin{array}{l}\text { with metformin in patients with Type } 2 \\
\text { diabetes inadequately controlled with } \\
\text { metformin alone: a 52-week, } \\
\text { randomized study. }\end{array}$ & & & \\
\hline $\begin{array}{l}\text { Comparison of vildagliptin and } \\
\text { pioglitazone in patients with type } 2 \\
\text { diabetes inadequately controlled with } \\
\text { metformin. }\end{array}$ & $\begin{array}{l}\text { Bolli,G.; Dotta,F.; } \\
\text { Colin,L.; Minic,B.; } \\
\text { Goodman,M. }\end{array}$ & $\begin{array}{l}\text { Diabetes, Obesity } \\
\text { and Metabolism }\end{array}$ & 3 \\
\hline $\begin{array}{l}\text { Dapagliflozin add-on to metformin in } \\
\text { type } 2 \text { diabetes inadequately controlled } \\
\text { with metformin: a randomized, double- } \\
\text { blind, placebo-controlled } 102 \text {-week trial }\end{array}$ & $\begin{array}{l}\text { Bailey, C. J.; Gross, J. } \\
\text { L.; Hennicken, D.; } \\
\text { lqbal, N.; Mansfield, T. } \\
\text { A.; List, J. F. }\end{array}$ & BMC Medicine & 3 \\
\hline $\begin{array}{l}\text { Dapagliflozin and insulin resistance in } \\
\text { patients with type } 2 \text { diabetes }\end{array}$ & Katz, A.; Yeh, H. & Diabetes & 3 \\
\hline $\begin{array}{l}\text { Dapagliflozin as Monotherapy or } \\
\text { Combination Therapy in Japanese } \\
\text { Patients with Type } 2 \text { Diabetes: an } \\
\text { Open-Label Study }\end{array}$ & $\begin{array}{l}\text { Kaku K; Maegawa H; } \\
\text { Tanizawa Y; Kiyosue } \\
\text { A; Ide Y; Tokudome T; } \\
\text { Hoshino Y; Yang J; } \\
\text { Langkilde AM }\end{array}$ & Diabetes Therapy & 1 \\
\hline $\begin{array}{l}\text { Dapagliflozin in type } 2 \text { diabetes: } \\
\text { Effectiveness across the spectrum of } \\
\text { disease and over time }\end{array}$ & $\begin{array}{l}\text { Parikh, S.; Wilding, J.; } \\
\text { Jabbour, S.; Hardy, E. }\end{array}$ & $\begin{array}{l}\text { International Journal } \\
\text { of Clinical Practice }\end{array}$ & 3 \\
\hline $\begin{array}{l}\text { Dapagliflozin produces long-term } \\
\text { reductions in body weight, waist } \\
\text { circumference and total fat mass in } \\
\text { patients with type } 2 \text { diabetes } \\
\text { inadequately controlled on metformin }\end{array}$ & $\begin{array}{l}\text { Bolinder, J.; Ljunggren, } \\
\text { Ã-; Johansson, L.; } \\
\text { Wilding, J. P. H.; } \\
\text { Langkilde, A. M.; } \\
\text { SjÃđstrÃđm, C. D.; } \\
\text { Sugg, J.; Parikh, S. }\end{array}$ & Diabetologia & 3 \\
\hline $\begin{array}{l}\text { Dapagliflozin, a sodium-glucose } \\
\text { cotransporter } 2 \text { inhibitor, has a low } \\
\text { propensity to cause hypoglycemia in } \\
\text { patients with type } 2 \text { diabetes }\end{array}$ & $\begin{array}{l}\text { Rohwedder, K.; Hruba, } \\
\text { V.; Salsali, A.; Ying, L. } \\
\text { H.; Sugg, J.; List, J. F.; } \\
\text { Parikh, S. J. }\end{array}$ & Diabetes & 3 \\
\hline $\begin{array}{l}\text { Dapagliflozin, a selective SGLT2 } \\
\text { inhibitor, has a low propensity to cause } \\
\text { hypoglycaemia in patients with type } 2 \\
\text { diabetes }\end{array}$ & $\begin{array}{l}\text { Rohwedder, K.; Hrub, } \\
\text { V.; Salsali, A.; Ying, L.; } \\
\text { Sugg, J.; List, J. F.; } \\
\text { Parikh, S. }\end{array}$ & Diabetologia & 3 \\
\hline $\begin{array}{l}\text { Diabetes-Related Quality Measure } \\
\text { Attainment: Canagliflozin Versus } \\
\text { Sitagliptin Based on a Pooled Analysis }\end{array}$ & $\begin{array}{l}\text { Bailey, Robert, A.; } \\
\text { Vijapurkar, Ujjwala; } \\
\text { Meininger, Gary, E.; }\end{array}$ & $\begin{array}{l}\text { American Journal of } \\
\text { Managed Care }\end{array}$ & 3 \\
\hline
\end{tabular}




\begin{tabular}{|c|c|c|c|}
\hline of 2 Clinical Trials. & $\begin{array}{l}\text { Rupnow, Marcia F. T.; } \\
\text { Blonde, Lawrence }\end{array}$ & & \\
\hline $\begin{array}{l}\text { A randomized, double-blind, placebo- } \\
\text { controlled trial evaluating sitagliptin } \\
\text { action on insulin resistance parameters } \\
\text { and beta-cell function. }\end{array}$ & $\begin{array}{l}\text { Derosa,G.; } \\
\text { Carbone,A.; } \\
\text { D'angelo,A.; Querci,F.; } \\
\text { Fogari,E.; Cicero,A. F.; } \\
\text { Maffioli,P. }\end{array}$ & $\begin{array}{l}\text { Expert opinion on } \\
\text { pharmacotherapy }\end{array}$ & 3 \\
\hline $\begin{array}{l}\text { Regardless of the degree of glycaemic } \\
\text { control, linagliptin has lower } \\
\text { hypoglycaemia risk than all doses of } \\
\text { glimepiride, at all time points, over the } \\
\text { course of a 2-year trial }\end{array}$ & $\begin{array}{l}\text { Gallwitz, B.; } \\
\text { Rosenstock, J.; Patel, } \\
\text { S.; von Eynatten, M.; } \\
\text { Hehnke, U.; } \\
\text { Mehlburger, L.; Dugi, } \\
\text { K. A.; Woerle, H. J. }\end{array}$ & $\begin{array}{l}\text { Diabetes, Obesity } \\
\text { and Metabolism }\end{array}$ & 3 \\
\hline $\begin{array}{l}\text { Regardless of the degree of glycemic } \\
\text { control, Linagliptin (LINA) has lower } \\
\text { hypoglycemia risk than all doses of } \\
\text { Glimepiride (GLIM), at all timeg points } \\
\text { over a 2-year trial }\end{array}$ & $\begin{array}{l}\text { Gallwitz, B.; } \\
\text { Rosenstock, J.; Patel, } \\
\text { S.; Von Eynatten, M.; } \\
\text { Hehnke, U.; } \\
\text { Mehlburger, L.; Dugi, } \\
\text { K. A.; Woerle, H. J. }\end{array}$ & $\begin{array}{l}\text { Diabetologie und } \\
\text { Stoffwechsel }\end{array}$ & 3 \\
\hline $\begin{array}{l}\text { Safety and efficacy of dulaglutide } \\
\text { versus sitagliptin after } 104 \text { weeks in } \\
\text { type } 2 \text { diabetes (award-5) }\end{array}$ & $\begin{array}{l}\text { Guerci, B.; Weinstock, } \\
\text { R.; Umpierrez, G.; } \\
\text { Nauck, M.; Boleyn, K.; } \\
\text { Skrivanek, Z.; } \\
\text { Milicevic, Z. }\end{array}$ & $\begin{array}{l}\text { Canadian Journal of } \\
\text { Diabetes }\end{array}$ & 3 \\
\hline $\begin{array}{l}\text { Safety and efficacy of dulaglutide vs } \\
\text { sitagliptin after } 104 \text { weeks in type } 2 \\
\text { diabetes (AWARD-5) }\end{array}$ & $\begin{array}{l}\text { Guerci, B.; Weinstock, } \\
\text { R. S.; Umpierrez, G.; } \\
\text { Nauck, M. A.; Boleyn, } \\
\text { K.; Skrivanek, Z.; } \\
\text { Milicevic, Z. }\end{array}$ & Diabetologia & 3 \\
\hline $\begin{array}{l}\text { Safety and efficacy of dulaglutide vs. } \\
\text { sitagliptin after } 104 \text { weeks in type } 2 \\
\text { diabetes (AWARD-5) }\end{array}$ & $\begin{array}{l}\text { Weinstock, R. S.; } \\
\text { Umpierrez, G. E.; } \\
\text { Guerci, B.; Nauck, M. } \\
\text { A.; Boleyn, K. L.; } \\
\text { Skrivanek, Z.; } \\
\text { Milicevic, Z. }\end{array}$ & Diabetes & 3 \\
\hline $\begin{array}{l}\text { Safety and efficacy of once-weekly } \\
\text { dulaglutide versus sitagliptin after } 2 \\
\text { years in metformin-treated patients with } \\
\text { type } 2 \text { diabetes (AWARD-5): a }\end{array}$ & $\begin{array}{l}\text { Weinstock, R. S.; } \\
\text { Guerci, B.; Umpierrez, } \\
\text { G.; Nauck, M. A.; } \\
\text { Skrivanek, Z.; } \\
\text { Milicevic, Z. }\end{array}$ & $\begin{array}{l}\text { Diabetes, Obesity } \\
\text { andMetabolism }\end{array}$ & 3 \\
\hline
\end{tabular}




\begin{tabular}{|c|c|c|c|}
\hline randomized, phase III study & & & \\
\hline $\begin{array}{l}\text { Safety and efficacy of treatment with } \\
\text { sitagliptin or glipizide in patients with } \\
\text { type } 2 \text { diabetes inadequately controlled } \\
\text { on metformin: a 2-year study }\end{array}$ & $\begin{array}{l}\text { Seck, T.; Nauck, M.; } \\
\text { Sheng, D.; Sunga, S.; } \\
\text { Davies, M. J.; Stein, P. } \\
\text { P.; Kaufman, K. D.; } \\
\text { Amatruda, J. M. }\end{array}$ & $\begin{array}{l}\text { International Journal } \\
\text { of Clinical Practice }\end{array}$ & 3 \\
\hline $\begin{array}{l}\text { Safety and tolerability of empagliflozin } \\
\text { (EMPA) in phase III trials and their } \\
\text { extensions in patients with type } 2 \\
\text { diabetes (T2DM) }\end{array}$ & $\begin{array}{l}\text { Roden, M.; Merker, L.; } \\
\text { Christiansen, A. V.; } \\
\text { Roux, F.; Hantel, S.; } \\
\text { Salsali, A.; Kim, G.; } \\
\text { Meinicke, T.; Lund, S. } \\
\text { S.; Hach, T.; Woerle, } \\
\text { H. J.; Broedl, U. C. }\end{array}$ & Diabetologia & 3 \\
\hline $\begin{array}{l}\text { Safety and tolerability of linagliptin in } \\
\text { patients with type } 2 \text { diabetes: A } \\
\text { comprehensive pooled analysis of } 22 \\
\text { placebo-controlled studies. }\end{array}$ & $\begin{array}{l}\text { Lehrke,M.; Marx,N.; } \\
\text { Patel,S.; Seck,T.; } \\
\text { Crowe,S.; Cheng,K.; } \\
\text { Von Eynatten,M.; } \\
\text { Johansen,O. E. }\end{array}$ & Clinical therapeutics & 3 \\
\hline $\begin{array}{l}\text { Safety and tolerability of sitagliptin in } \\
\text { clinical studies: A pooled analysis of } \\
\text { data from 10,246 patients with type } 2 \\
\text { diabetes }\end{array}$ & $\begin{array}{l}\text { Williams-Herman, D.; } \\
\text { Engel, S. S.; Round, } \\
\text { E.; Johnson, J.; Golm, } \\
\text { G. T.; Guo, H.; Musser, } \\
\text { B. J.; Davies, M. J.; } \\
\text { Kaufman, K. D.; } \\
\text { Goldstein, B. J. }\end{array}$ & $\begin{array}{l}\text { BMC Endocrine } \\
\text { Disorders }\end{array}$ & 3 \\
\hline $\begin{array}{l}\text { Safety and tolerability of sitagliptin in } \\
\text { patients with type } 2 \text { diabetes: a pooled } \\
\text { analysis }\end{array}$ & \multicolumn{2}{|c|}{$\begin{array}{l}\text { Williams-Herman, D.; Round, E.; Swern, A. S.; } \\
\text { Musser, B.; Davies, M. J.; Stein, P. P.; } \\
\text { Kaufman, K. D.; Amatruda, J. M. }\end{array}$} & 3 \\
\hline $\begin{array}{l}\text { Safety and tolerability of sitagliptin in } \\
\text { type } 2 \text { diabetes: pooled analysis of } 25 \\
\text { clinical studies }\end{array}$ & \multicolumn{2}{|c|}{$\begin{array}{l}\text { Engel, S. S.; Round, E.; Golm, G. T.; } \\
\text { Kaufman, K. D.; Goldstein, B. J. }\end{array}$} & 3 \\
\hline $\begin{array}{l}\text { Safety of linagliptin in } 8,778 \text { patients } \\
\text { with type } 2 \text { diabetes mellitus: Pooled } \\
\text { analysis of } 23 \text { placebo-controlled, } \\
\text { randomized clinical trials }\end{array}$ & $\begin{array}{l}\text { Schernthaner, G.; } \\
\text { Khunti, K.; Patel, S.; } \\
\text { Cheng, K.; Mattheus, } \\
\text { M.; Woerle, H. J. }\end{array}$ & Diabetes & 3 \\
\hline $\begin{array}{l}\text { Safety of linagliptin in } 8778 \text { patients } \\
\text { with type } 2 \text { diabetes mellitus: Pooled } \\
\text { analysis of } 23 \text { placebo-controlled } \\
\text { randomised clinical trials }\end{array}$ & $\begin{array}{l}\text { Schernthaner, G.; } \\
\text { Khunti, K.; Patel, S.; } \\
\text { Cheng, K.; Mattheus, } \\
\text { M.; Woerle, H. J. }\end{array}$ & Diabetologia & 3 \\
\hline
\end{tabular}




\begin{tabular}{|c|c|c|c|}
\hline $\begin{array}{l}\text { Safety of saxagliptin: events of special } \\
\text { interest in } 9156 \text { patients with type } 2 \\
\text { diabetes mellitus }\end{array}$ & $\begin{array}{l}\text { Hirshberg, B.; Parker, } \\
\text { A.; Edelberg, H.; } \\
\text { Donovan, M.; Iqbal, N. }\end{array}$ & $\begin{array}{l}\text { Diabetes/Metabolism } \\
\text { Research and } \\
\text { Reviews }\end{array}$ & 3 \\
\hline $\begin{array}{l}\text { Safety profile of saxagliptin (SAXA) in } \\
\text { combination with } 2 \text { other agents: Data } \\
\text { from dual-therapy trials in patients } \\
\text { receiving rescue treatment }\end{array}$ & $\begin{array}{l}\text { Allen, E.; Karyekar, C.; } \\
\text { Ã-hman, P. }\end{array}$ & Diabetes & 3 \\
\hline $\begin{array}{l}\text { Safety of sitagliptin in elderly patients } \\
\text { with type } 2 \text { diabetes: A pooled analysis } \\
\text { of } 25 \text { clinical studies }\end{array}$ & $\begin{array}{l}\text { Round, E. M.; Engel, } \\
\text { S. S.; Golm, G. T.; } \\
\text { Davies, M. J.; } \\
\text { Kaufman, K. D.; } \\
\text { Goldstein, B. J. }\end{array}$ & Drugs and Aging & 3 \\
\hline $\begin{array}{l}\text { Saxagliptin (SAXA) efficacy and safety } \\
\text { in patients with type } 2 \text { diabetes mellitus } \\
\text { (T2DM) stratified by cardiovascular } \\
\text { (CV) risk factors }\end{array}$ & $\begin{array}{l}\text { Cook, W.; Bryzinski, } \\
\text { B.; Allen, E.; } \\
\text { Hirshberg, B. }\end{array}$ & Endocrine Reviews. & 3 \\
\hline $\begin{array}{l}\text { Saxagliptin (SAXA) vs Glipizide (GLIP) } \\
\text { as add-on therapy to metformin (MET) } \\
\text { for type } 2 \text { diabetes (T2D): Assessment } \\
\text { of HbA1c, hypoglycemia, and weight } \\
\text { gain }\end{array}$ & $\begin{array}{l}\text { Bouzamondo, H.; } \\
\text { Karyekar, C.; Berglind, } \\
\text { N.; Allen, E. }\end{array}$ & Diabetes & 3 \\
\hline $\begin{array}{l}\text { Saxagliptin efficacy and safety in } \\
\text { patients with type } 2 \text { diabetes mellitus } \\
\text { stratified by cardiovascular disease } \\
\text { history and cardiovascular risk factors: } \\
\text { analysis of } 3 \text { clinical trials }\end{array}$ & $\begin{array}{l}\text { Cook, W.; Minervini, } \\
\text { G.; Bryzinski, B.; } \\
\text { Hirshberg, B. }\end{array}$ & Postgrad Med & 3 \\
\hline $\begin{array}{l}\text { Distribution of weight loss with } \\
\text { canagliflozin in patients with type } 2 \\
\text { diabetes mellitus over } 104 \text { weeks }\end{array}$ & $\begin{array}{l}\text { StenlÃđf, K.; Blonde, } \\
\text { L.; Fung, A.; Xie, J.; } \\
\text { Canovatchel, W.; } \\
\text { Meininger, G. }\end{array}$ & Diabetologia & 3 \\
\hline $\begin{array}{l}\text { Durability of the efficacy and safety of } \\
\text { alogliptin compared to glipizide over } 2 \\
\text { years when used in combination with } \\
\text { metformin }\end{array}$ & $\begin{array}{l}\text { Del Prato, S.; } \\
\text { Camisasca, R.; Wilson, } \\
\text { C.; Wilson, C.; Fleck, } \\
\text { P. }\end{array}$ & Diabetologia & 3 \\
\hline $\begin{array}{l}\text { Durability of the efficacy and safety of } \\
\text { alogliptin compared to glipizide when } \\
\text { used in combination with metformin: } 1 \\
\text { year interim analysis }\end{array}$ & $\begin{array}{l}\text { Del Prato, S.; } \\
\text { Camisasca, R.; Wilson, } \\
\text { C.; Fleck, P. }\end{array}$ & Diabetes & 3 \\
\hline Durability of the efficacy and safety of & Del Prato, S.; & Diabetes, Obesity & 3 \\
\hline
\end{tabular}




\begin{tabular}{|c|c|c|c|}
\hline $\begin{array}{l}\text { alogliptin compared with glipizide in } \\
\text { type } 2 \text { diabetes mellitus: a } 2 \text {-year study }\end{array}$ & $\begin{array}{l}\text { Camisasca, R.; Wilson, } \\
\text { C.; Fleck, P. }\end{array}$ & andMetabolism & \\
\hline $\begin{array}{l}\text { Early onset of increased hypoglycemic } \\
\text { incidence with glipizide (GLIP) vs. } \\
\text { saxagliptin (SAXA) in type } 2 \text { diabetes } \\
\text { patients on metformin }\end{array}$ & $\begin{array}{l}\text { Frederich, R.; Parikh, } \\
\text { S. J.; Allen, E.; } \\
\text { Bryzinski, B.; Cook, } \\
\text { W.; Hirshberg, B. }\end{array}$ & Diabetes & 3 \\
\hline $\begin{array}{l}\text { Effects of a combination of sitagliptin } \\
\text { plus metformin vs metformin } \\
\text { monotherapy on glycemic control, ?-cell } \\
\text { function and insulin resistance in type } 2 \\
\text { diabetic patients }\end{array}$ & $\begin{array}{l}\text { Derosa, G.; Carbone, } \\
\text { A.; Franzetti, I.; Querci, } \\
\text { F.; Fogari, E.; Bianchi, } \\
\text { L.; Bonaventura, A.; } \\
\text { Romano, D.; Cicero, A. } \\
\text { F.; Maffioli, P. }\end{array}$ & $\begin{array}{l}\text { Diabetes Research } \\
\text { and Clinical Practice }\end{array}$ & 3 \\
\hline $\begin{array}{l}\text { The efficacy and effectiveness in } \\
\mathrm{HbA} 1 \mathrm{c}-\text { lowering is dependent on } \\
\text { baseline body mass index (BMI) for } \\
\text { sitagliptin but not canagliflozin in the } \\
\text { treatment of type } 2 \text { diabetes mellitus } \\
\text { (T2DM) }\end{array}$ & $\begin{array}{l}\text { Diels, J.; Angermund, } \\
\text { R.; Schroeder, M.; } \\
\text { Worbes-Cerezo, M.; } \\
\text { Thompson, G. }\end{array}$ & Value in Health & 3 \\
\hline $\begin{array}{l}\text { Efficacy and safety of alogliptin added } \\
\text { to metformin in Japanese patients with } \\
\text { type } 2 \text { diabetes: A randomized, double- } \\
\text { blind, placebo-controlled trial with an } \\
\text { open-label, long-term extension study. }\end{array}$ & $\begin{array}{l}\text { Seino,Y.; Miyata,Y.; } \\
\text { Hiroi,S.; Hirayama,M.; } \\
\text { Kaku,K. }\end{array}$ & $\begin{array}{l}\text { Diabetes, Obesity } \\
\text { and Metabolism }\end{array}$ & 3 \\
\hline $\begin{array}{l}\text { Efficacy and safety of anagliptin } \\
\text { compared with sitagliptin as an add-on } \\
\text { for patients with type } 2 \text { diabetes } \\
\text { inadequately controlled with metformin } \\
\text { monotherapy }\end{array}$ & $\begin{array}{l}\text { Jin, S. M.; Park, S. W.; } \\
\text { Yoon, K. H.; Lee, M. K. }\end{array}$ & $\begin{array}{l}\text { Diabetes research } \\
\text { and clinical practice }\end{array}$ & 3 \\
\hline $\begin{array}{l}\text { Efficacy and safety of Canagliflozin in } \\
\text { subjects with type } 2 \text { diabetes mellitus } \\
\text { on background metformin }\end{array}$ & $\begin{array}{l}\text { Januszewicz, A.; } \\
\text { Lavalle GonzÃ̈lez, F.; } \\
\text { Davidson, J.; Qiu, R.; } \\
\text { Tong, C.; Hamilton, G.; } \\
\text { Meininger, G. }\end{array}$ & $\begin{array}{l}\text { Diabetologie und } \\
\text { Stoffwechsel }\end{array}$ & 3 \\
\hline $\begin{array}{l}\text { Efficacy and safety of canagliflozin } \\
\text { versus glimepiride in patients with type } \\
2 \text { diabetes inadequately controlled with } \\
\text { metformin (CANTATA-SU): } 52 \text { week } \\
\text { results from a randomised, double- } \\
\text { blind, phase } 3 \text { non-inferiority trial }\end{array}$ & $\begin{array}{l}\text { Cefalu, W. T.; Leiter, L. } \\
\text { A.; Yoon, K. H.; Arias, } \\
\text { P.; Niskanen, L.; Xie, } \\
\text { J.; Balis, D. A.; } \\
\text { Canovatchel, W.; } \\
\text { Meininger, G. }\end{array}$ & The Lancet & 3 \\
\hline
\end{tabular}




\begin{tabular}{|c|c|c|c|}
\hline $\begin{array}{l}\text { Efficacy and safety of canagliflozin, a } \\
\text { sodium glucose co-transporter } 2 \\
\text { inhibitor, compared with glimepiride in } \\
\text { patients with type } 2 \text { diabetes on } \\
\text { background metformin }\end{array}$ & $\begin{array}{l}\text { Niskanen, L.; Cefalu, } \\
\text { W. T.; Leiter, L. A.; Xie, } \\
\text { J.; Balis, D.; } \\
\text { Canovatchel, W.; } \\
\text { Meininger, G. }\end{array}$ & Diabetologia & 3 \\
\hline $\begin{array}{l}\text { Efficacy and safety of canagliflozin, a } \\
\text { sodium glucose co-transporter } 2 \\
\text { inhibitor, versus glimepiride in patients } \\
\text { with type } 2 \text { diabetes on background } \\
\text { metformin }\end{array}$ & $\begin{array}{l}\text { Leiter, L. A.; Cefalu, W. } \\
\text { T.; Niskanen, L.; Xie, } \\
\text { J.; Millington, D.; } \\
\text { Canovatchel, W.; } \\
\text { Meininger, G. }\end{array}$ & $\begin{array}{l}\text { Canadian Journal of } \\
\text { Diabetes }\end{array}$ & 3 \\
\hline $\begin{array}{l}\text { Efficacy and safety of dulaglutide } \\
\text { versus sitagliptin after } 52 \text { weeks in type } \\
2 \text { diabetes (award-5) }\end{array}$ & $\begin{array}{l}\text { Nauck, M.; Weinstock, } \\
\text { R.; Umpierrez, G.; } \\
\text { Guerci, B.; Boleyn, K.; } \\
\text { Skrivanek, Z.; } \\
\text { Milicevic, Z. }\end{array}$ & $\begin{array}{l}\text { Canadian Journal of } \\
\text { Diabetes }\end{array}$ & 3 \\
\hline $\begin{array}{l}\text { Efficacy and safety of dulaglutide } \\
\text { versus sitagliptin after } 52 \text { weeks in type } \\
2 \text { diabetes in a randomized controlled } \\
\text { trial (AWARD-5) }\end{array}$ & $\begin{array}{l}\text { Nauck, M.; Weinstock, } \\
\text { R. S.; Umpierrez, G. } \\
\text { E.; Guerci, B.; } \\
\text { Skrivanek, Z.; } \\
\text { Milicevic, Z. }\end{array}$ & Diabetes Care & 3 \\
\hline $\begin{array}{l}\text { Efficacy and safety of dulaglutide vs. } \\
\text { Sitagliptin after } 52 \text { weeks in type } 2 \\
\text { diabetes (AWARD-5) }\end{array}$ & $\begin{array}{l}\text { Nauck, M. A.; } \\
\text { Weinstock, R. S.; } \\
\text { Umpierrez, G. E.; } \\
\text { Guerci, B.; Boleyn, K.; } \\
\text { Skrivanek, Z.; } \\
\text { Milicevic, Z. }\end{array}$ & Diabetes & 3 \\
\hline $\begin{array}{l}\text { Efficacy and safety of ipragliflozin } \\
\text { (IPRA) in Japanese patients with type } 2 \\
\text { diabetes mellitus (T2DM) stratified by } \\
\text { Body Mass Index (BMI) }\end{array}$ & $\begin{array}{l}\text { Kashiwagi, A.; } \\
\text { Yoshida, S.; } \\
\text { Nakamura, I.; Kazuta, } \\
\text { K.; Ueyama, E.; } \\
\text { Takahashi, H.; Satomi, } \\
\text { H.; Kosakai, Y.; } \\
\text { Kawamuki, K. }\end{array}$ & Diabetes & 3 \\
\hline $\begin{array}{l}\text { Efficacy and safety of luseogliflozin } \\
\text { added to various oral antidiabetic drugs } \\
\text { in Japanese patients with type } 2 \\
\text { diabetes mellitus }\end{array}$ & $\begin{array}{l}\text { Seino, Y.; Inagaki, N.; } \\
\text { Haneda, M.; Kaku, K.; } \\
\text { Sasaki, T.; Fukatsu, A.; } \\
\text { Ubukata, M.; Sakai, S.; } \\
\text { Samukawa, Y. }\end{array}$ & J Diabetes Invest & 2 \\
\hline $\begin{array}{l}\text { Efficacy and safety of saxagliptin in } \\
\text { patients with type } 2 \text { diabetes mellitus }\end{array}$ & $\begin{array}{l}\text { Minervini, G.; Cook, } \\
\text { W.; Bryzinski, B.; }\end{array}$ & Endocrine Practice & 3 \\
\hline
\end{tabular}




\begin{tabular}{|c|c|c|c|}
\hline $\begin{array}{l}\text { and cardiovascular disease history or } \\
\text { cardiovascular risk factors }\end{array}$ & Hirshberg, B. & & \\
\hline $\begin{array}{l}\text { Efficacy and safety of switching from } \\
\text { the DPP-4 inhibitor sitagliptin to the } \\
\text { human GLP-1 analog liraglutide after } 52 \\
\text { weeks in metformin-treated patients } \\
\text { with type } 2 \text { diabetes a randomized, } \\
\text { open-label trial. }\end{array}$ & $\begin{array}{l}\text { Pratley,R. E.; } \\
\text { Nauck,M. A.; Bailey,T.; } \\
\text { Montanya,E.; Filetti,S.; } \\
\text { Garber,A. J.; } \\
\text { Thomsen,A. B.; } \\
\text { Furber,S.; Davies,M. }\end{array}$ & Diabetes care & 3 \\
\hline $\begin{array}{l}\text { Efficacy and safety of taspoglutide } \\
\text { versus sitagliptin for type } 2 \text { diabetes } \\
\text { mellitus (T-Emerge } 4 \text { Trial) }\end{array}$ & $\begin{array}{l}\text { Bergenstal, R. M.; } \\
\text { Forti, A.; Chiasson, J. } \\
\text { L.; Woloschak, M.; } \\
\text { Boldrin, M.; Balena, R. }\end{array}$ & Diabetes Therapy & 3 \\
\hline $\begin{array}{l}\text { Efficacy and safety of the dipeptidyl } \\
\text { peptidase- } 4 \text { inhibitor, sitagliptin, } \\
\text { compared with the sulfonylurea, } \\
\text { glipizide, in patients with type } 2 \\
\text { diabetes inadequately controlled on } \\
\text { metformin alone: a randomized, double- } \\
\text { blind, non-inferiority trial }\end{array}$ & $\begin{array}{l}\text { Nauck, M. A.; } \\
\text { Meininger, G.; Sheng, } \\
\text { D.; Terranella, L.; } \\
\text { Stein, P. P. }\end{array}$ & $\begin{array}{l}\text { Diabetes, Obesity } \\
\text { and Metabolism }\end{array}$ & 3 \\
\hline $\begin{array}{l}\text { Efficacy and tolerability of saxagliptin } \\
\text { compared with glimepiride in elderly } \\
\text { patients with type } 2 \text { diabetes: a } \\
\text { randomized, controlled study } \\
\text { (GENERATION) }\end{array}$ & $\begin{array}{l}\text { Schernthaner, G.; } \\
\text { Duran-Garcia, S.; } \\
\text { Hanefeld, M.; Langslet, } \\
\text { G.; Niskanen, L.; } \\
\text { Ostgren, C. J.; } \\
\text { Malvolti, E.; Hardy, E. }\end{array}$ & $\begin{array}{l}\text { Diabetes, Obesity } \\
\text { and Metabolism }\end{array}$ & 3 \\
\hline $\begin{array}{l}\text { Efficacy of add-on vildagliptin therapy to } \\
\text { metformin for type ii diabetes mellitus } \\
\text { patients in South Indian resource } \\
\text { limited settings-pilot study }\end{array}$ & $\begin{array}{l}\text { Vigneshwaran, E.; } \\
\text { Venkatesh, K.; } \\
\text { Kirankumar, C.; } \\
\text { Rubiya, S.; } \\
\text { Padmanabhareddy, Y.; } \\
\text { Achar, B. }\end{array}$ & Value in Health & 2 \\
\hline $\begin{array}{l}\text { Efficacy of saxagliptin as an add-on to } \\
\text { oral monotherapy in the phase } 3 \text { clinical } \\
\text { development program: predictive } \\
\text { factors of the treatment response in } \\
\text { type } 2 \text { diabetes }\end{array}$ & $\begin{array}{l}\text { Gautier, J. F.; } \\
\text { Sauvanet, J. P. }\end{array}$ & $\begin{array}{l}\text { Ann Endocrinol } \\
\text { (Paris) }\end{array}$ & 3 \\
\hline $\begin{array}{l}\text { Empagliflozin as add-on to metformin in } \\
\text { patients with Type } 2 \text { diabetes }\end{array}$ & $\begin{array}{l}\text { Merker, L.; Haring, H. } \\
\text { U.; Vedel Christiansen, } \\
\text { A.; Roux, F.; Salsali, } \\
\text { A.; Kim, G.; Meinicke, }\end{array}$ & Diabetic Medicine & 3 \\
\hline
\end{tabular}




\begin{tabular}{|c|c|c|c|}
\hline & $\begin{array}{l}\text { T.; Woerle, H. J.; } \\
\text { Broedl, U. C. }\end{array}$ & & \\
\hline $\begin{array}{l}\text { Evaluation of the positive effects on } \\
\text { insulin-resistance and beta-cell } \\
\text { measurements of vildagliptin in addition } \\
\text { to metformin in type } 2 \text { diabetic patients }\end{array}$ & $\begin{array}{l}\text { Derosa, G.; Ragonesi, } \\
\text { P. D.; Carbone, A.; } \\
\text { Fogari, E.; D'Angelo, } \\
\text { A.; Cicero, A. F.; } \\
\text { Maffioli, P. }\end{array}$ & $\begin{array}{l}\text { Pharmacological } \\
\text { Research }\end{array}$ & 3 \\
\hline $\begin{array}{l}\text { Fifty-two-week efficacy and safety of } \\
\text { vildagliptin vs. glimepiride in patients } \\
\text { with type } 2 \text { diabetes mellitus } \\
\text { inadequately controlled on metformin } \\
\text { monotherapy }\end{array}$ & $\begin{array}{l}\text { Ferrannini, E.; } \\
\text { Fonseca, V.; Zinman, } \\
\text { B.; Matthews, D.; } \\
\text { Ahren, B.; Byiers, S.; } \\
\text { Shao, Q.; Dejager, S. }\end{array}$ & $\begin{array}{l}\text { Diabetes, Obesity } \\
\text { and Metabolism }\end{array}$ & 3 \\
\hline $\begin{array}{l}\text { Fixed dose combinations of } \\
\text { empagliflozin/linagliptin for } 52 \text { weeks as } \\
\text { add-on to metformin in subjects with } \\
\text { type } 2 \text { diabetes }\end{array}$ & $\begin{array}{l}\text { Patel, S.; DeFronzo, } \\
\text { R.; Lewin, A.; Liu, D.; } \\
\text { Kaste, R.; Woerle, H. } \\
\text { J.; Broedl, U. C. }\end{array}$ & Diabetologia & 3 \\
\hline $\begin{array}{l}\text { Further improvement in glycaemic } \\
\text { control and weight loss observed in } \\
\text { patients with Type } 2 \text { diabetes switched } \\
\text { from the DPP-4 inhibitor sitagliptin to } \\
\text { the human GLP-1 analogue liraglutide }\end{array}$ & $\begin{array}{l}\text { Matthews, D. R.; } \\
\text { Pratley, R. E.; Nauck, } \\
\text { M. A.; Bailey, T.; } \\
\text { Montanya, E.; Davies, } \\
\text { M. J.; Filetti, S.; } \\
\text { Garber, A. J.; } \\
\text { Thomsen, A. B. }\end{array}$ & Diabetic medicine & 3 \\
\hline $\begin{array}{l}\text { The GLP-1 analogue liraglutide } \\
\text { provides more effective glycaemic } \\
\text { control and weight reduction over one } \\
\text { year compared with sitagliptin, a DPP-4 } \\
\text { inhibitor, In patients with Type } 2 \\
\text { diabetes inadequately controlled with } \\
\text { metformin }\end{array}$ & $\begin{array}{l}\text { Davies, M.; Nauck, M.; } \\
\text { Bailey, T.; Montanya, } \\
\text { E.; Cuddihy, R.; Filetti, } \\
\text { S.; Garber, A.; } \\
\text { Thomsen, A. B.; } \\
\text { Hartvig, H.; Pratley, R. }\end{array}$ & Diabetic medicine & 3 \\
\hline $\begin{array}{l}\text { Saxagliptin efficacy and safety in } \\
\text { patients with type } 2 \text { diabetes receiving } \\
\text { concomitant statin therapy }\end{array}$ & $\begin{array}{l}\text { Bryzinski, B.; Allen, E.; } \\
\text { Cook, W.; Hirshberg, } \\
\text { B. }\end{array}$ & $\begin{array}{l}\text { Journal of Diabetes } \\
\text { and Its } \\
\text { Complications }\end{array}$ & 3 \\
\hline $\begin{array}{l}\text { Saxagliptin is non-inferior to glipizide in } \\
\text { patients with type } 2 \text { diabetes mellitus } \\
\text { inadequately controlled on metformin } \\
\text { alone: a } 52 \text {-week randomised controlled } \\
\text { trial. }\end{array}$ & $\begin{array}{l}\text { Goke, B; Gallwitz, B; } \\
\text { Eriksson, J; Hellqvist, } \\
\text { A; Gause-Nilsson, I; } \\
\text { D1680C00001 } \\
\text { Investigators }\end{array}$ & $\begin{array}{l}\text { International journal } \\
\text { of clinical practice }\end{array}$ & 3 \\
\hline Saxagliptin is non-inferior to glipizide & GÃTke, B.; Gallwitz, & Diabetes & 3 \\
\hline
\end{tabular}




\begin{tabular}{|c|c|c|c|}
\hline $\begin{array}{l}\text { when added to metformin in patients } \\
\text { with type } 2 \text { diabetes mellitus }\end{array}$ & $\begin{array}{l}\text { B.; Eriksson, J.; } \\
\text { Hellqvist, A.; Gause- } \\
\text { Nilsson, I. }\end{array}$ & & \\
\hline $\begin{array}{l}\text { Saxagliptin is similar in glycaemic } \\
\text { variability more effective in metabolic } \\
\text { control than acarbose in aged type } 2 \\
\text { diabetes inadequately controlled with } \\
\text { metformin }\end{array}$ & $\begin{array}{l}\text { Wang, M. M.; Lin, S.; } \\
\text { Chen, Y. M.; Shu, J.; } \\
\text { Lu, H. Y.; Zhang, Y. J.; } \\
\text { Xie, R. Y.; Zeng, L. Y.; } \\
\text { Mu, P. W. }\end{array}$ & $\begin{array}{l}\text { Diabetes research } \\
\text { and clinical practice }\end{array}$ & 3 \\
\hline $\begin{array}{l}\text { Saxagliptin versus glipizide as add-on } \\
\text { therapy to metformin: assessment of } \\
\text { hypoglycemia }\end{array}$ & $\begin{array}{l}\text { Mintz, M. L.; Minervini, } \\
\text { G. }\end{array}$ & Curr Med Res Opin & 3 \\
\hline $\begin{array}{l}\text { Saxagliptin vs glipizide as add-on } \\
\text { therapy to metformin in patients with } \\
\text { type } 2 \text { diabetes: A 2-year assessment } \\
\text { of HbA1c, hypoglycaemia, and weight } \\
\text { gain in a randomised, double-blind } \\
\text { study }\end{array}$ & Allen, E.; Berglind, N. & Diabetologia & 3 \\
\hline $\begin{array}{l}\text { Saxagliptin vs. glipizide as add-on } \\
\text { therapy in patients with type } 2 \text { diabetes } \\
\text { mellitus inadequately controlled on } \\
\text { metformin alone: long-term (52-week) } \\
\text { extension of a 52-week randomised } \\
\text { controlled trial }\end{array}$ & $\begin{array}{l}\text { Goke, B.; Gallwitz, B.; } \\
\text { Eriksson, J. G.; } \\
\text { Hellqvist, A.; Gause- } \\
\text { Nilsson, I. }\end{array}$ & Int J Clin Pract & 3 \\
\hline $\begin{array}{l}\text { Saxagliptin vs glipizide as add-on } \\
\text { therapy to metformin for type } 2 \text { diabetes } \\
\text { mellitus (T2DM): Long-term safety and } \\
\text { efficacy }\end{array}$ & $\begin{array}{l}\text { Goke, B.; Gallwitz, B.; } \\
\text { Eriksson, J.; } \\
\text { Bokelundsingh, S.; } \\
\text { Gause-Nilsson, I. }\end{array}$ & Diabetes & 3 \\
\hline $\begin{array}{l}\text { Simultaneous reduction in both } \mathrm{HbA} 1 \mathrm{c} \\
\text { and body weight with canagliflozin } \\
\text { compared with glimepiride in metformin- } \\
\text { treated patients with type } 2 \text { diabetes } \\
\text { mellitus over } 104 \text { weeks }\end{array}$ & $\begin{array}{l}\text { Langslet, G.; Leiter, L. } \\
\text { A.; Vijapurkar, U.; } \\
\text { Davies, M.; } \\
\text { Canovatchel, W. }\end{array}$ & Diabetologia & 3 \\
\hline $\begin{array}{l}\text { Sitagliptin (SITA) provides similar } \\
\text { glycemic control with weight loss and } \\
\text { less hypoglycemia compared to } \\
\text { sulfonylurea (SU) in older patients with } \\
\text { type } 2 \text { diabetes (T2DM) }\end{array}$ & $\begin{array}{l}\text { Seck, T.; Engel, S. S.; } \\
\text { Chen, Y.; Golm, G. T.; } \\
\text { Davies, M. J.; } \\
\text { Kaufman, K. D.; } \\
\text { Goldstein, B. }\end{array}$ & Diabetes & 3 \\
\hline $\begin{array}{l}\text { Sitagliptin more effectively achieves a } \\
\text { composite endpoint for } \mathrm{A} 1 \mathrm{C} \text { reduction, }\end{array}$ & $\begin{array}{l}\text { Seck, T. L.; Engel, S. } \\
\text { S.; Williams-Herman, }\end{array}$ & $\begin{array}{l}\text { Diabetes Research } \\
\text { and Clinical Practice }\end{array}$ & 3 \\
\hline
\end{tabular}




\begin{tabular}{|c|c|c|c|}
\hline $\begin{array}{l}\text { lack of hypoglycemia and no body } \\
\text { weight gain compared with glipizide }\end{array}$ & $\begin{array}{l}\text { D. E.; Sisk, C. M.; } \\
\text { Golm, G. T.; Wang, H.; } \\
\text { Kaufman, K. D.; } \\
\text { Goldstein, B. J. }\end{array}$ & & \\
\hline $\begin{array}{l}\text { Sitagliptin provides similar glycaemic } \\
\text { improvement with less hypoglycaemia } \\
\text { in the elderly with type } 2 \text { diabetes } \\
\text { mellitus compared to sulphonylurea }\end{array}$ & $\begin{array}{l}\text { Shankar, R.; Engel, S. } \\
\text { S.; Xu, L.; Golm, G. T.; } \\
\text { Davies, M. J.; } \\
\text { Kaufman, K. D.; } \\
\text { Goldstein, B. J. }\end{array}$ & Diabetologia & 3 \\
\hline $\begin{array}{l}\text { Sitagliptin provides similar glycemic } \\
\text { improvement with less hypoglycemia in } \\
\text { the elderly with type } 2 \text { diabetes mellitus } \\
\text { compared to sulfonylurea }\end{array}$ & $\begin{array}{l}\text { Shankar, R.; Engel, S. } \\
\text { S.; Xu, L.; Golm, G. T.; } \\
\text { Davies, M. J.; } \\
\text { Kaufman, K. D.; } \\
\text { Goldstein, B. J. }\end{array}$ & Diabetes & 3 \\
\hline $\begin{array}{l}\text { Slope of decline in } \mathrm{HbA} 1 \mathrm{c} \text { with } \\
\text { empagliflozin appears to be greater } \\
\text { than the slope with sitagliptin or } \\
\text { glimepiride in patients with type } 2 \\
\text { diabetes (T2DM) }\end{array}$ & $\begin{array}{l}\text { Defronzo, R. A.; } \\
\text { Elsasser, U.; Lund, S. } \\
\text { S.; Hach, T. }\end{array}$ & Diabetes & 3 \\
\hline $\begin{array}{l}\text { Sustained efficacy of dapagliflozin when } \\
\text { added to metformin in type } 2 \text { diabetes } \\
\text { inadequately controlled by metformin } \\
\text { monotherapy }\end{array}$ & $\begin{array}{l}\text { Bailey, C. J.; Gross, J. } \\
\text { L.; Yadav, M.; Iqbal, } \\
\text { N.; Mansfield, T. A.; } \\
\text { List, J. F. }\end{array}$ & Diabetologia & 3 \\
\hline $\begin{array}{l}\text { Sustained reductions in weight and } \\
\mathrm{HbA} 1 \mathrm{c} \text { with dapagliflozin: Long-term } \\
\text { results from phase III clinical studies in } \\
\text { type } 2 \text { diabetes }\end{array}$ & $\begin{array}{l}\text { Bailey, C. J.; Wilding, } \\
\text { J.; Nauck, M. A.; } \\
\text { Ferrannini, E.; } \\
\text { Ptaszynska, A. A.; } \\
\text { Apanovitch, A. M.; } \\
\text { Sugg, J.; Parikh, S. J. }\end{array}$ & Diabetologia & 3 \\
\hline $\begin{array}{l}\text { Switching from the DPP-4 inhibitor } \\
\text { sitagliptin to the human GLP-1 analog } \\
\text { liraglutide further improves glycemic } \\
\text { control and weight loss in patients with } \\
\text { type } 2 \text { diabetes }\end{array}$ & $\begin{array}{l}\text { Pratley, R.; Nauck, M.; } \\
\text { Bailey, T.; Montanya, } \\
\text { E.; Filetti, S.; Garber, } \\
\text { A.; Thomsen, A. B.; } \\
\text { Hartvig, H.; Davies, M. }\end{array}$ & Diabetes & 3 \\
\hline $\begin{array}{l}\text { A greater proportion of people with } \\
\text { Type } 2 \text { diabetes, inadequately } \\
\text { controlled on metformin, reach the } \\
\text { composite target of } \mathrm{HbA} 1 \mathrm{c}<7.5 \% \\
(59 \mathrm{mmol} / \mathrm{mol} \text { ) and } \mathrm{a} \% \text { } ¥ 3 \% \text { body weight } \\
\text { reduction when treated with either }\end{array}$ & $\begin{array}{l}\text { Stein, L.; Schroeder, } \\
\text { M.; Diels, J.; Hamilton, } \\
\text { G.; Canovatchel, W. }\end{array}$ & Diabetic medicine & 3 \\
\hline
\end{tabular}




\begin{tabular}{|c|c|c|c|}
\hline $\begin{array}{l}\text { canagliflozin } 100 \mathrm{mg} \text { or } 300 \mathrm{mg} \\
\text { compared with sitagliptin } 100 \mathrm{mg} \text { at } 52 \\
\text { weeks }\end{array}$ & & & \\
\hline $\begin{array}{l}\text { Health-related quality of life (EQ-5D) } \\
\text { among type } 2 \text { diabetes mellitus patients } \\
\text { treated with dapagliflozin over } 2 \text { years }\end{array}$ & $\begin{array}{l}\text { Grandy, S.; Langkilde, } \\
\text { A. M.; Sugg, J. E.; } \\
\text { Parikh, S.; } \\
\text { SjÃ IstrÃđm, C. D. }\end{array}$ & $\begin{array}{l}\text { International journal } \\
\text { of clinical practice }\end{array}$ & 3 \\
\hline $\begin{array}{l}\text { Higher Risk of Hypoglycemia with } \\
\text { Glimepiride Versus Vildagliptin in } \\
\text { Patients with Type } 2 \text { Diabetes is not } \\
\text { Driven by High Doses of Glimepiride: } \\
\text { Divergent Patient Susceptibilities? }\end{array}$ & $\begin{array}{l}\text { Ahren B; Foley JE; } \\
\text { Dejager S; Akacha M; } \\
\text { Shao Q; Heimann G; } \\
\text { Dworak M; Schweizer } \\
\text { A }\end{array}$ & Diabetes Therapy & 3 \\
\hline $\begin{array}{l}\text { Impact of treatment with saxagliptin on } \\
\text { glycaemic stability and } \hat{I}^{2} \text {-cell function in } \\
\text { the SAVOR-TIMI } 53 \text { study }\end{array}$ & $\begin{array}{l}\text { Leibowitz, G.; Cahn, } \\
\text { A.; Bhatt, D. L.; } \\
\text { Hirshberg, B.; } \\
\text { Mosenzon, O.; Wei, C.; } \\
\text { Jermendy, G.; Sheu, } \\
\text { W. H. H.; Sendon, J. } \\
\text { L.; Im, K.; Braunwald, } \\
\text { E.; Scirica, B. M.; Raz, } \\
\text { I. }\end{array}$ & $\begin{array}{l}\text { Diabetes, Obesity } \\
\text { and Metabolism }\end{array}$ & 2 \\
\hline $\begin{array}{l}\text { Improved meal-related beta-cell } \\
\text { function and insulin sensitivity by the } \\
\text { dipeptidyl peptidase-IV inhibitor } \\
\text { vildagliptin in metformin-treated patients } \\
\text { with type } 2 \text { diabetes over } 1 \text { year }\end{array}$ & $\begin{array}{l}\text { AhrÃ@n, B.; Pacini, G.; } \\
\text { Foley, J. E.; } \\
\text { Schweizer, A. }\end{array}$ & Diabetes Care & 3 \\
\hline $\begin{array}{l}\text { Improved meal-related insulin } \\
\text { processing contributes to the } \\
\text { enhancement of B-cell function by the } \\
\text { DPP-4 inhibitor vildagliptin in patients } \\
\text { with type } 2 \text { diabetes. }\end{array}$ & $\begin{array}{l}\text { Ahren,B.; Pacini,G.; } \\
\text { Tura,A.; Foley,J. E.; } \\
\text { Schweizer,A. }\end{array}$ & $\begin{array}{l}\text { Hormone and } \\
\text { Metabolic Research }\end{array}$ & 3 \\
\hline $\begin{array}{l}\text { Improvement in postprandial glucose } \\
\text { after 2-year treatment with alogliptin in } \\
\text { patients with type } 2 \text { diabetes } \\
\text { inadequately controlled with metformin }\end{array}$ & $\begin{array}{l}\text { Liu, J.; Oh, R.; Wilson, } \\
\text { C.; Camisasca, R. }\end{array}$ & Diabetologia & 3 \\
\hline $\begin{array}{l}\text { Ipragliflozin in combination with } \\
\text { metformin for the treatment of } \\
\text { Japanese patients with type } 2 \text { diabetes: } \\
\text { ILLUMINATE, a randomized, double- } \\
\text { blind, placebo-controlled study }\end{array}$ & $\begin{array}{l}\text { Kashiwagi, A.; Kazuta, } \\
\text { K.; Goto, K.; Yoshida, } \\
\text { S.; Ueyama, E.; } \\
\text { Utsuno, A. }\end{array}$ & $\begin{array}{l}\text { Diabetes, Obesity } \\
\text { and Metabolism }\end{array}$ & 3 \\
\hline
\end{tabular}




\begin{tabular}{|c|c|c|c|}
\hline $\begin{array}{l}\text { Linagliptin has similar efficacy to } \\
\text { glimepiride but improved cardiovascular } \\
\text { safety over } 2 \text { years in patients with type } \\
2 \text { diabetes inadequately controlled on } \\
\text { metformin }\end{array}$ & $\begin{array}{l}\text { Gallwitz, B.; Uhlig- } \\
\text { Laske, B.; } \\
\text { Bhattacharaya, S.; } \\
\text { Patel, S.; Dugi, K. A.; } \\
\text { Woerle, H. J. }\end{array}$ & $\begin{array}{l}\text { Diabetes, } \\
\text { Stoffwechsel und } \\
\text { Herz }\end{array}$ & 3 \\
\hline $\begin{array}{l}\text { Linagliptin is more effective than } \\
\text { glimepiride at achieving a composite } \\
\text { outcome of A1C target with no } \\
\text { hypoglycemia and no weight gain over } \\
2 \text { years in mildly hyperglycemic T2D pts } \\
\text { on metformin }\end{array}$ & $\begin{array}{l}\text { Gallwitz, B.; } \\
\text { Rosenstock, J.; Emser, } \\
\text { A.; Von Eynatten, M.; } \\
\text { Woerle, H. J. }\end{array}$ & Diabetes & 3 \\
\hline $\begin{array}{l}\text { Linagliptin is more effective than } \\
\text { glimepiride at achieving a composite } \\
\text { outcome of target } \mathrm{HbA} \text { ?c }<7 \% \text { with no } \\
\text { hypoglycaemia and no weight gain over } \\
2 \text { years }\end{array}$ & $\begin{array}{l}\text { Gallwitz, B.; } \\
\text { Rosenstock, J.; Emser, } \\
\text { A.; Eynatten, M.; } \\
\text { Woerle, H. J. }\end{array}$ & $\begin{array}{l}\text { International Journal } \\
\text { of Clinical Practice }\end{array}$ & 3 \\
\hline $\begin{array}{l}\text { Linagliptin provides effective, well- } \\
\text { tolerated add-on therapy to pre-existing } \\
\text { oral antidiabetic therapy over } 1 \text { year in } \\
\text { Japanese patients with type } 2 \text { diabetes }\end{array}$ & $\begin{array}{l}\text { Inagaki, N.; Watada, } \\
\text { H.; Murai, M.; } \\
\text { Kagimura, T.; Gong, } \\
\text { Y.; Patel, S.; Woerle, } \\
\text { H. J. }\end{array}$ & $\begin{array}{l}\text { Diabetes, Obesity } \\
\text { and Metabolism }\end{array}$ & 3 \\
\hline $\begin{array}{l}\text { Liraglutide treatment for } 1 \text { year offers } \\
\text { sustained and more effective glycemic } \\
\text { control and weight reduction compared } \\
\text { with sitagliptin, both in combination with } \\
\text { metformin, in patients with type } 2 \\
\text { diabetes }\end{array}$ & $\begin{array}{l}\text { Pratley, R.; Nauck, M.; } \\
\text { Bailey, T.; Montanya, } \\
\text { E.; Cuddihy, R.; Filetti, } \\
\text { S.; Garber, A.; } \\
\text { Thomsen, A.; Hartvig, } \\
\text { H.; Davies, M. }\end{array}$ & Diabetes & 3 \\
\hline $\begin{array}{l}\text { Liraglutide versus sitagliptin for patients } \\
\text { with type } 2 \text { diabetes who did not have } \\
\text { adequate glycaemic control with } \\
\text { metformin: A } 26 \text {-week, randomised, } \\
\text { parallel-group, open-label trial }\end{array}$ & $\begin{array}{l}\text { Pratley, R. E.; Nauck, } \\
\text { M.; Bailey, T.; } \\
\text { Montanya, E.; } \\
\text { Cuddihy, R.; Filetti, S.; } \\
\text { Thomsen, A. B.; } \\
\text { Sondergaard, R. E.; } \\
\text { Davies, M. }\end{array}$ & Lancet & 3 \\
\hline $\begin{array}{l}\text { Long-term efficacy of sitagliptin as } \\
\text { either monotherapy or add-on therapy } \\
\text { to metformin: improvement in glycemic } \\
\text { control over } 2 \text { years in patients with } \\
\text { type } 2 \text { diabetes }\end{array}$ & $\begin{array}{l}\text { Katzeff, H. L.; } \\
\text { Williams-Herman, D.; } \\
\text { Xu, L.; Golm, G. T.; } \\
\text { Wang, H.; Dong, Q.; } \\
\text { Johnson, J. R.; O'Neill, } \\
\text { E. A.; Kaufman, K. D.; }\end{array}$ & $\begin{array}{l}\text { Current Medical } \\
\text { Research and } \\
\text { Opinion }\end{array}$ & 3 \\
\hline
\end{tabular}




\begin{tabular}{|c|c|c|c|}
\hline & $\begin{array}{l}\text { Engel, S. S.; Goldstein, } \\
\text { B. J. }\end{array}$ & & \\
\hline $\begin{array}{l}\text { Long-term safety and efficacy of } \\
\text { empagliflozin, sitagliptin, and metformin }\end{array}$ & $\begin{array}{l}\text { Ferrannini, E.; Berk, } \\
\text { A.; Hantel, S.; Pinnetti, } \\
\text { S.; Hach, T.; Woerle, } \\
\text { H. J.; Broedl, U. C. }\end{array}$ & Diabetes Care & 3 \\
\hline $\begin{array}{l}\text { Long-term safety and efficacy of } \\
\text { linagliptin as monotherapy or in } \\
\text { combination with other oral glucose- } \\
\text { lowering agents in } 2121 \text { subjects with } \\
\text { type } 2 \text { diabetes: up to } 2 \text { years exposure } \\
\text { in } 24 \text {-week phase III trials followed by a } \\
78 \text {-week open-label extension }\end{array}$ & $\begin{array}{l}\text { Gomis, R.; Owens, D. } \\
\text { R.; Taskinen, M. R.; } \\
\text { Del Prato, S.; Patel, S.; } \\
\text { Pivovarova, A.; } \\
\text { Schlosser, A.; Woerle, } \\
\text { H. J. }\end{array}$ & Int J Clin Pract & 3 \\
\hline $\begin{array}{l}\text { Long-term safety and efficacy of the } \\
\text { DPP-4 inhibitor linagliptin: Data from a } \\
\text { large } 2 \text {-year study in subjects with type } \\
2 \text { diabetes mellitus }\end{array}$ & $\begin{array}{l}\text { Schlosser, A.; Owens, } \\
\text { D.; Taskinen, M. R.; } \\
\text { Del Prato, S.; Gomis, } \\
\text { R.; Patel, S.; } \\
\text { Pivovarova, A.; } \\
\text { Woerle, H. J. }\end{array}$ & Diabetologia & 3 \\
\hline $\begin{array}{l}\text { Long-term safety and tolerability of } \\
\text { saxagliptin add-on therapy in older } \\
\text { patients (aged â\%o } 65 \text { years) with type } \\
2 \text { diabetes }\end{array}$ & $\begin{array}{l}\text { Iqbal, N.; Allen, E.; } \tilde{A}_{-} \\
\text {hman, P. }\end{array}$ & $\begin{array}{l}\text { Clinical Interventions } \\
\text { in Aging }\end{array}$ & 3 \\
\hline $\begin{array}{l}\text { Long-term tolerability of saxagliptin as } \\
\text { add-on therapy in type } 2 \text { diabetes } \\
\text { (T2D): Pooled analysis }\end{array}$ & $\begin{array}{l}\text { Allen, E.; Fleming, D.; } \\
\text { Berglind, N.; Donovan, } \\
\text { M.; Ã-hman, P. }\end{array}$ & Diabetes & 3 \\
\hline $\begin{array}{l}\text { Long-term treatment with empagliflozin } \\
\text { as add-on to oral antidiabetes therapy } \\
\text { in Japanese patients with type } 2 \\
\text { diabetes mellitus }\end{array}$ & $\begin{array}{l}\text { Araki, E.; Tanizawa, } \\
\text { Y.; Tanaka, Y.; } \\
\text { Taniguchi, A.; Koiwai, } \\
\text { K.; Kim, G.; Salsali, A.; } \\
\text { Woerle, H. J.; Broedl, } \\
\text { U. C. }\end{array}$ & $\begin{array}{l}\text { Diabetes, Obesity } \\
\text { and Metabolism }\end{array}$ & 3 \\
\hline $\begin{array}{l}\text { Lower risk of hypoglycemia with } \\
\text { sitagliptin compared to glipizide when } \\
\text { either is added to metformin therapy: a } \\
\text { pre-specified analysis adjusting for the } \\
\text { most recently measured } \mathrm{HbA}(1 \mathrm{c}) \text { value }\end{array}$ & $\begin{array}{l}\text { Krobot, K. J.; Ferrante, } \\
\text { S. A.; Davies, M. J.; } \\
\text { Seck, T.; Meininger, G. } \\
\text { E.; Williams-Herman, } \\
\text { D.; Kaufman, K. D.; } \\
\text { Goldstein, B. J. }\end{array}$ & $\begin{array}{l}\text { Current Medical } \\
\text { Research and } \\
\text { Opinion }\end{array}$ & 3 \\
\hline One year of liraglutide treatment offers & Pratley, R.; Nauck, M.; & International Journal & 3 \\
\hline
\end{tabular}




\begin{tabular}{|c|c|c|c|}
\hline $\begin{array}{l}\text { sustained and more effective glycaemic } \\
\text { control and weight reduction compared } \\
\text { with sitagliptin, both in combination with } \\
\text { metformin, in patients with type } 2 \\
\text { diabetes: A randomised, parallel-group, } \\
\text { open-label trial }\end{array}$ & $\begin{array}{l}\text { Bailey, T.; Montanya, } \\
\text { E.; Cuddihy, R.; Filetti, } \\
\text { S.; Garber, A.; } \\
\text { Thomsen, A. B.; } \\
\text { Hartvig, H.; Davies, M. }\end{array}$ & of Clinical Practice & \\
\hline $\begin{array}{l}\text { [Oral add-on therapy to metformin in } \\
\text { type } 2 \text { diabetes mellitus: a direct } \\
\text { comparison between canagliflozin and } \\
\text { sitagliptin] }\end{array}$ & Schnell, O. & $\begin{array}{l}\text { Dtsch Med } \\
\text { Wochenschr }\end{array}$ & 1 \\
\hline $\begin{array}{l}\text { Patient-reported outcomes (PRO) from } \\
\text { a } 104 \text { week, phase } 3 \text {, randomised, } \\
\text { placebo-controlled study comparing } \\
\text { once weekly dulaglutide to sitagliptin } \\
\text { and placebo in metformin-treated } \\
\text { patients with Type } 2 \text { diabetes; the } \\
\text { Assessment of Weekly Administration } \\
\text { of Dulaglutide in Diabetes (AWARD-5) } \\
\text { trial }\end{array}$ & $\begin{array}{l}\text { Reaney, M.; Yu, M.; } \\
\text { Adetunji, O.; Milicevic, } \\
\text { Z. }\end{array}$ & Diabetic Medicine & 3 \\
\hline $\begin{array}{l}\text { Predisposing factors for hypoglycaemia: } \\
\text { Analysis from the SAVOR-TIMI } 53 \text { trial }\end{array}$ & $\begin{array}{l}\text { Raz, I.; Cahn, A.; } \\
\text { Scirica, B. M.; Ray, K. } \\
\text { K.; Goldberg } \\
\text { Eliaschewitz, F.; } \\
\text { Moses, R. G.; } \\
\text { Hirshberg, B.; } \\
\text { Sjostrand, M.; Iqbal, } \\
\text { N.; Mosenzon, O.; } \\
\text { Lewis, B. S.; Bhatt, D. } \\
\text { L. }\end{array}$ & Diabetologia & 3 \\
\hline $\begin{array}{l}\text { Predisposing factors for hypoglycemia: } \\
\text { Analysis from the savortimi } 53 \text { trial }\end{array}$ & $\begin{array}{l}\text { Raz, I.; Cahn, A.; } \\
\text { Scirica, B. M.; } \\
\text { Eliaschewitz, F.; } \\
\text { Moses, R.; Hirshberg, } \\
\text { B.; SjÃđIstrand, M.; } \\
\text { Iqbal, N.; Mosenzon, } \\
\text { O.; Lewis, B. S.; Bhatt, } \\
\text { D. L. }\end{array}$ & Diabetes & 3 \\
\hline $\begin{array}{l}\text { Twelve- and 52-week efficacy of the } \\
\text { dipeptidyl peptidase IV inhibitor LAF237 } \\
\text { in metformin-treated patients with type }\end{array}$ & $\begin{array}{l}\text { AhrÃ@n, B.; Gomis, R.; } \\
\text { Standl, E.; Mills, D.; } \\
\text { Schweizer, A. }\end{array}$ & Diabetes Care & 3 \\
\hline
\end{tabular}




\begin{tabular}{|c|c|c|c|}
\hline 2 diabetes & & & \\
\hline $\begin{array}{l}\text { Variations in inflammatory biomarkers } \\
\text { following the addition of sitagliptin in } \\
\text { patients with type } 2 \text { diabetes not } \\
\text { controlled with metformin. }\end{array}$ & $\begin{array}{l}\text { Derosa,G.; } \\
\text { Carbone,A.; } \\
\text { D'Angelo,A.; Querci,F.; } \\
\text { Fogari,E.; Cicero,A. F. } \\
\text { G.; Maffioli,P. }\end{array}$ & Internal Medicine & 3 \\
\hline $\begin{array}{l}\text { Vildagliptin action on some } \\
\text { adipocytokine levels in type } 2 \text { diabetic } \\
\text { patients: A 12-month, placebo- } \\
\text { controlled study. }\end{array}$ & $\begin{array}{l}\text { Derosa,G.; } \\
\text { Ragonesi,P. D.; } \\
\text { Carbone,A.; Fogari,E.; } \\
\text { D'Angelo,A.; Cicero,A. } \\
\text { F.; Maffioli,P. }\end{array}$ & $\begin{array}{l}\text { Expert opinion on } \\
\text { pharmacotherapy }\end{array}$ & 3 \\
\hline $\begin{array}{l}\text { Vildagliptin added to metformin on beta- } \\
\text { cell function after a euglycemic } \\
\text { hyperinsulinemic and hyperglycemic } \\
\text { clamp in type } 2 \text { diabetes patients. }\end{array}$ & $\begin{array}{l}\text { Derosa,G.; } \\
\text { Ragonesi,P. D.; } \\
\text { Carbone,A.; Fogari,E.; } \\
\text { Bianchi,L.; } \\
\text { Bonaventura,A.; } \\
\text { Romano,D.; Cicero,A. } \\
\text { F. G.; Maffioli,P. }\end{array}$ & $\begin{array}{l}\text { Diabetes } \\
\text { Technology and } \\
\text { Therapeutics }\end{array}$ & 3 \\
\hline $\begin{array}{l}\text { Vildagliptin add-on to metformin } \\
\text { produces similar efficacy and reduced } \\
\text { hypoglycaemic risk compared with } \\
\text { glimepiride, with no weight gain: results } \\
\text { from a 2-year study. }\end{array}$ & $\begin{array}{l}\text { Matthews, D R; } \\
\text { Dejager, S; Ahren, B; } \\
\text { Fonseca, V; } \\
\text { Ferrannini, E; } \\
\text { Couturier, A; Foley, J } \\
\text { E; Zinman, B }\end{array}$ & $\begin{array}{l}\text { Diabetes, obesity \& } \\
\text { metabolism }\end{array}$ & 3 \\
\hline $\begin{array}{l}\text { Vildagliptin more effectively achieves a } \\
\text { composite endpoint of } \mathrm{HbA}(1) \mathrm{c}<7.0 \% \\
\text { without hypoglycaemia and weight gain } \\
\text { compared with glimepiride after } 2 \text { years } \\
\text { of treatment }\end{array}$ & $\begin{array}{l}\text { Bader, G.; Geransar, } \\
\text { P.; Schweizer, A. }\end{array}$ & $\begin{array}{l}\text { Diabetes Research } \\
\text { and Clinical Practice }\end{array}$ & 3 \\
\hline $\begin{array}{l}\text { Weight- and health-related quality of life } \\
\text { (WRQoL and HRQoL) with canagliflozin } \\
\text { (CANA) versus sitagliptin (SITA) in } \\
\text { subjects with type } 2 \text { diabetes mellitus } \\
\text { (T2DM) on background metformin }\end{array}$ & Traina, S. B.; Ho, K. F. & Value in Health & 3 \\
\hline $\begin{array}{l}\text { Weight loss related quality of life among } \\
\text { type } 2 \text { diabetes mellitus patients treated } \\
\text { with dapagliflozin }\end{array}$ & $\begin{array}{l}\text { Parikh, S. J.; } \\
\text { Ingelgard, A.; } \\
\text { Langkilde, A. M.; Sugg, } \\
\text { J.; Grandy, S. }\end{array}$ & Diabetologia & 3 \\
\hline Weight loss-related quality of life among & Grandy, S.; & Diabetes & 3 \\
\hline
\end{tabular}




\begin{tabular}{|c|c|c|c|}
\hline $\begin{array}{l}\text { type } 2 \text { diabetes mellitus (T2DM) } \\
\text { patients treated with dapagliflozin }\end{array}$ & $\begin{array}{l}\text { IngelgaËšrd, A.; } \\
\text { Langkilde, A.; Sugg, J. } \\
\text { E.; Parikh, S. J. }\end{array}$ & & \\
\hline $\begin{array}{l}\text { Canagliflozin provides durable glycemic } \\
\text { improvements and body weight } \\
\text { reduction over } 104 \text { weeks versus } \\
\text { glimepiride in patients with type } 2 \\
\text { diabetes on metformin: a randomized, } \\
\text { double-blind, phase } 3 \text { study }\end{array}$ & $\begin{array}{l}\text { Leiter, L. A.; Yoon, K. } \\
\text { H.; Arias, P.; Langslet, } \\
\text { G.; Xie, J.; Balis, D. A.; } \\
\text { Millington, D.; } \\
\text { Vercruysse, F.; } \\
\text { Canovatchel, W.; } \\
\text { Meininger, G. }\end{array}$ & Diabetes Care & 3 \\
\hline $\begin{array}{l}\text { Empagliflozin as add-on to metformin in } \\
\text { people with Type } 2 \text { diabetes }\end{array}$ & $\begin{array}{l}\text { Merker, L.; HÃaring, H. } \\
\text { U.; Christiansen, A. V.; } \\
\text { Roux, F.; Salsali, A.; } \\
\text { Kim, G.; Meinicke, T.; } \\
\text { Woerle, H. J.; Broedl, } \\
\text { U. C. }\end{array}$ & Diabetic Medicine & 3 \\
\hline $\begin{array}{l}\text { Long-term efficacy and safety of } \\
\text { canagliflozin over } 104 \text { weeks in patients } \\
\text { aged } 55-80 \text { years with type } 2 \text { diabetes }\end{array}$ & $\begin{array}{l}\text { Bode, B.; StenlÃđf, K.; } \\
\text { Harris, S.; Sullivan, D.; } \\
\text { Fung, A.; Usiskin, K.; } \\
\text { Meininger, G. }\end{array}$ & $\begin{array}{l}\text { Diabetes Obes } \\
\text { Metab }\end{array}$ & 3 \\
\hline
\end{tabular}


Anexo G. Resumo das características basais, desenho dos estudos, intervenção e desfechos.

\begin{tabular}{|c|c|c|}
\hline Estudo / droga de interesse & Participantes e características basais & Intervenção/desfechos \\
\hline $\begin{array}{l}\text { DAPA - Dapaglifozina } \\
\text { Del Parto et al (110) } \\
\text { Estudo multicêntrico }(\mathrm{n}=77) \\
\text { Registro ClinicalTrials: } \\
\text { NCT00660907 } \\
\text { Desenho: } 2 \text { braços, duplo cego, } \\
\text { randomizado, dapagliflozina 2,5- } \\
10 \mathrm{mg} \text {, comparador glipizida 5- } \\
20 \mathrm{mg} \\
\text { Duração: dois anos com } \\
\text { extensão de dois anos }\end{array}$ & $\begin{array}{l}\text { N: } 814 \\
\text { Participantes: pacientes com } \\
\text { descompensado em uso de metformina pré- } \\
\text { randomização }(\geq 1500 \mathrm{mg} / \mathrm{d}) \text { ou ADO prévio } \\
\text { suspenso e titulação metformina } \\
\text { Idade (anos): dapa+met } 58 \pm 9 \text {; glipizida+met } 59 \\
\pm 10 \\
\text { HbA1c (\%): dapa+met } 7,65 \pm 0,9 \text {; glipizida+met } \\
7,74 \pm 0,9 \\
\text { Peso (Kg): sem valores disponíveis }\end{array}$ & $\begin{array}{l}\text { Intervenção: dapagliflozina } 2,5-10 \mathrm{mg} \\
\text { Comparador: glipizida } 5-20 \text { mg } \\
\text { Desfecho primário: variação média nos } \\
\text { níveis de HbA1c em } 52 \text { semanas } \\
\text { Desfechos secundários: variação peso } \\
\text { em } 52 \text { semanas, proporção de pacientes } \\
\text { com pelo menos } 1 \text { episódio de } \\
\text { hipoglicemia, pacientes com redução de } \\
\text { peso maior que } 5 \%\end{array}$ \\
\hline $\begin{array}{l}\text { EMPA - Empagliflozina } \\
\text { Salsali et al (111) } \\
\text { Estudo multicêntrico ( } \mathrm{n}=182) \\
\text { Registro ClinicalTrials: } \\
\text { NCT01167881 } \\
\text { Desenho: } 2 \text { braços, duplo cego, } \\
\text { randomizado, empagliflozina } \\
25 \mathrm{mg} \text {, comparador glimepirida } \\
\text { 1-4 mg } \\
\text { Duração: dois anos com } \\
\text { extensão de dois anos }\end{array}$ & $\begin{array}{l}\text { N: } 1549 \\
\text { Participantes: pacientes com } \\
\text { descompensado em uso de metformina }(\geq 1500 \\
\text { mg/d). } \\
\text { Idade (anos): empa+met } 56,2 \pm 10 \text {; glimep+met } \\
55,7 \pm 10 \\
\text { HbA1c (\%): empa+met } 7,92 \pm 0,8 \text {; glimep+met } \\
7,92 \pm 0,9 \\
\text { Peso (Kg): empa+met } 82,5 \pm 19 \text {; glimep+met } 83 \pm \\
19\end{array}$ & $\begin{array}{l}\text { Intervenção: empagliflozina } 25 \mathrm{mg} \\
\text { Comparador: glimepirida 1-4 mg } \\
\text { Desfecho primário: variação média nos } \\
\text { níveis de HbA1c em } 104 \text { semanas } \\
\text { Desfechos secundários: variação do } \\
\text { peso em } 104 \text { semanas, variação pressão } \\
\text { arterial sistólica e diastólica em } 104 \\
\text { semanas }\end{array}$ \\
\hline $\begin{array}{l}\text { SAXA - Saxagliptina } \\
\text { Rosenstock et al }(122) \\
\text { Estudo multicêntrico }(n=154) \\
\text { Registro ClinicalTrials: } \\
\text { NCT00121667 } \\
\text { Desenho: } 4 \text { braços, duplo cego, } \\
\text { randomizado, saxagliptina 2,5, } 5\end{array}$ & $\begin{array}{l}\text { N: } 743 \\
\text { Participantes: pacientes com } \quad \text { DM2 } \\
\text { descompensado em uso de metformina }(\geq 1500 \\
\text { mg/d). } \\
\text { Idade (anos): saxa+met } 54,7 \pm 9 ; \text { placebo+met } \\
54,8 \pm 10 \\
\text { HbA1c (\%): saxa+met } 8,07 \pm 0,1 ; \text { placebo+met }\end{array}$ & $\begin{array}{l}\text { Intervenção: saxagliptina } 5 \mathrm{mg} \\
\text { Comparador: placebo } \\
\text { Desfecho primário: variação média nos } \\
\text { níveis de HbA1c em } 25 \text { semanas } \\
\text { Desfechos secundários: variação } \\
\text { glicemia de jejum em } 24 \text { semanas, } \\
\text { porcentagem de pacientes que atingiram }\end{array}$ \\
\hline
\end{tabular}




\begin{tabular}{|c|c|c|}
\hline $\begin{array}{l}\text { e } 10 \mathrm{mg} \text {, comparador placebo. } \\
\text { Droga de resgate pioglitazona } \\
15-45 \mathrm{mg} \text {. Incluído na análise } \\
\text { somente a dose de } 5 \mathrm{mg} \text {. } \\
\text { Duração: quatro anos }\end{array}$ & $\begin{array}{l}8,06 \pm 0,1 \\
\text { Peso }(\mathbf{K g}) \text { : sem valores disponíveis }\end{array}$ & $\mathrm{HbA} 1 \mathrm{c}<7$ em 24 semanas \\
\hline $\begin{array}{l}\text { SITA - Sitagliptina } \\
\text { Matthews et al }(124) \\
\text { Estudo multicêntrico }(\mathrm{n}=386) \\
\text { Registro ClinicalTrials: } \\
\text { NCT00838903 } \\
\text { Desenho: } 4 \text { braços, duplo cego, } \\
\text { randomizado, albiglutida 30-50 } \\
\mathrm{mg} \text {, comparadores sitagliptina } \\
100 \mathrm{mg} \text {, glimepirida 2-4 mg e } \\
\text { placebo. } \\
\text { Duração: três anos }\end{array}$ & $\begin{array}{l}\text { N: } 1012 \\
\text { Participantes: pacientes com DM2 } \\
\text { descompensado em uso de metformina }(\geq 1500 \\
\text { mg/d). } \\
\text { Idade (anos): albi+met } 54,3 \pm 10 \text {; sita+met } 54,3 \pm \\
9,8 \text {, glimep+met } 54,4 \pm 10 \text {, placebo+met } 56,1 \pm \\
10 \\
\text { HbA1c (\%): albi+met } 8,09 \pm 0,8 \text {; sita+met } 8,05 \pm \\
0,8 \text {, glimep+met } 8,12 \pm 0,84, \text { placebo+met } 8,15 \\
\pm 0,9 \\
\text { Peso (Kg): albi+met } 91,8 \pm 20 \text {; sita+met } 90,3 \pm \\
19, \text { glimep+met } 89,6 \pm 18, \text { placebo+met } 91,6 \pm 19\end{array}$ & $\begin{array}{l}\text { Intervenção: albiglutida } 30-50 \mathrm{mg} \\
\text { Comparadores: sitagliptina } 100 \mathrm{mg} \text {, } \\
\text { glimepirida } 2-4 \mathrm{mg} \text { e placebo } \\
\text { Desfecho primário: variação média nos } \\
\text { níveis de HbA1c em } 104 \text { semanas } \\
\text { Desfechos secundários: variação } \\
\text { HbA1c em } 156 \text { semanas, alteração da } \\
\text { glicemia de jejum em } 104 \text { e } 156 \text { semanas }\end{array}$ \\
\hline
\end{tabular}




\title{
Anexo H. Titulação das drogas e definição de falência e resgate terapêutico
}

\author{
Titulação das drogas e definição de falência e resgate terapêutico
}

\begin{tabular}{|l|l|}
\hline Titulação da droga no estudo & $\begin{array}{l}\text { Definição de falência terapêutica - resgate } \\
\text { com terceira droga }\end{array}$ \\
\hline
\end{tabular}

DAPA

Todos os pacientes iniciaram no nível I dapagliflozina na dose de $2,5 \mathrm{mg}$ ou glipizida na dose de $5 \mathrm{mg}$. Durante 18 semanas em um intervalo de $21 / 21$ dias, as doses foram tituladas para cima se a glicemia plasmática de jejum foi $\geq 180 \mathrm{mg} / \mathrm{dL}$. No nível II as doses foram de dapagliflozina $5 \mathrm{mg}$ e glipizida $10 \mathrm{mg}$ no nível III dapagliflozina $10 \mathrm{mg}$ e glipizida $20 \mathrm{mg}$. A titulação foi realizada até a dose máxima possível tolerada. Após 18 semanas de titulação, os pacientes entraram em um período de estabilidade da dose por 34 semanas, só permitindo a titulação para baixo. Após a semana 104 uma única titulação para cima foi permitida caso $\mathrm{HbA} 1 \mathrm{c}>7 \%$.

Pacientes com controle glicêmico inadequado durantes as primeiras 52 semanas de tratamento duplo cego foram considerados falha de tratamento por critério de específico no decorrer do tratamento. Após receber a máxima dose titulável ou tolerável por pelo menos 2 semanas, foram descontinuados se (1) glicemia de jejum $>270 \mathrm{mg} / \mathrm{dL}$ na semana 3,6 ou 9 ; (2) > $239 \mathrm{mg} / \mathrm{dL}$ na semana 12,15 ou 18 ; (3) $>220 \mathrm{mg} / \mathrm{dL}$ na semana 26 ou 34 ou (4) $>200 \mathrm{mg} / \mathrm{dL}$ na semana 42. Para o período de extensão de 104 semanas, um ajuste único para cima foi permitido se $\mathrm{HbA} 1 \mathrm{c}>7 \%$. No período de 104 a 208 semanas, o resgate foi realizado caso $\mathrm{HbA} 1 \mathrm{c} \geq 8$ e na avaliação do pesquisador se entre 7 e $8 \%$; não foi permitida titulação para cima, somente para baixo.

\section{EMPA}

Paciente receberam empagliflozina $25 \mathrm{mg}$ ou Necessidade de terapia de resgate caso glicemia de jejum confirmada $>240 \mathrm{mg} / \mathrm{dL}$ durante a semana 1 a $12,>200$ glimepirida com dose inicial de $1 \mathrm{mg}$, com titulação por glicemia capilar. Com aumento se glicemia $>110$ $\mathrm{mg} / \mathrm{dL}$ para $2 \mathrm{mg}$ na semana 4 , para $3 \mathrm{mg}$ na semana 8 e para a dose máxima de $4 \mathrm{mg} / \mathrm{d}$ na semana 12 . A $\mathrm{mg} / \mathrm{dL}$ durante as semanas 12 a 28 e $>180 \mathrm{mg} / \mathrm{dL}$ (ou HbA1c $>8 \%$ ) após a semana 28

titulação foi suspensa se o paciente apresentava risco de hipoglicemia. Caso hipoglicemia, a dose poderia ser titulada para baixo a qualquer tempo.

\section{SAXA}

Sem titulação

Pacientes nos grupos saxagliptina ou placebo receberam pioglitazona até a dose de 30 ou $45 \mathrm{mg} / \mathrm{d}$, não sendo excluídos do estudo. O critério utilizado foi glicose plasmática de jejum > $240 \mathrm{mg} / \mathrm{dL}$ na semana 4 e $6,>222 \mathrm{mg} / \mathrm{dL}$ na semana $8,>202 \mathrm{mg} / \mathrm{dL}$ na semana $12-24$, $\mathrm{HbA} 1 \mathrm{c}>8 \%$ na semana $30-50,>7,5 \%$ na semana $65-76$ e $>7 \%$ na semana 89-193. Pacientes com hiperglicemia persistente após 12 semanas da dose máxima da medicação de resgate descontinuaram o estudo.

\section{SITA}

Entre dia 1 e $<$ semana 2 sem titulação, $\geq$ semana 2 e < semana 4 sem titulação, semana 4 glicemia plasmática de jejum $\geq 250 \mathrm{mg} / \mathrm{dL}$ e HbA1c inalterada ou maior que a basal, > semana 4 e < semana 12 glicemia plasmática de jejum $\geq 250 \mathrm{mg} / \mathrm{dL}$ e HbA1c inalterada ou maior que a basal, $\geq$ semana $12 \mathrm{e}<$ semana $24 \mathrm{HbA} 1 \mathrm{c} \geq 7,5 \%$ e $\leq 0,5 \%$ de redução da linha de sabe, $\geq$ semana $24 \mathrm{e}<$ semana $104 \mathrm{HbA} 1 \mathrm{c} \geq$ $7,5 \%$, $\geq$ semana 104 e < semana $143 \mathrm{HbA} 1 \mathrm{c} \geq 7,5 \%$ e depois sem titulação. A dose inicial de glimepirida foi de $2 \mathrm{mg}$, titulável até $4 \mathrm{mg}$ e dose inicial da albiglutida foi de $30 \mathrm{mg}$, titulável até $50 \mathrm{mg}$.
Entre dia $1 \mathrm{e}<$ semana 2 sem resgaste $\geq$ semana $2 \mathrm{e}<$ semana 4 glicemia plasmática de jejum maior $280 \mathrm{mg} / \mathrm{dL}$ semana 4 glicemia plasmática de jejum maior $280 \mathrm{mg} / \mathrm{dL}$, > semana 4 e $<$ semana 12 glicemia plasmática de jejum $\geq$ $250 \mathrm{mg} / \mathrm{dL}$ com titulação previa por mais de 4 semanas, $\geq$ semana 12 e < semana $24 \mathrm{HbA} 1 \mathrm{c} \geq 8,5 \%$ e $\leq 0,5 \%$ de redução da linha de sabe com titulação previa por mais de 4 semanas, $\geq$ semana 24 e < semana $104 \mathrm{HbA} 1 \mathrm{c} \geq 8,5 \%$ com titulação previa por mais de 4 semanas, $\geq$ semana $104 \mathrm{e}<$ semana $143 \mathrm{HbA} 1 \mathrm{c} \geq 8 \%$ com titulação previa por mais de 4 semanas e $\geq$ semana 143 e $<$ semana $156 \mathrm{HbA} 1 \mathrm{c} \geq 8 \%$ com titulação previa por mais de 4 semanas. 


\title{
Anexo I. Definição de hipoglicemia por estudo
}

\author{
Definição de hipoglicemia por estudo
}

\section{DAPA}

1) Hipoglicemia maior - definido como um episódio sintomático de hipoglicemia com necessidade de ajuda externa e glicose capilar ou plasmática menor de $54 \mathrm{mg} / \mathrm{dL}$ e necessidade de glicose ou glucagon. 2) Hipoglicemia menor - evento de hipoglicemia sintomática ou não com glicose capilar ou plasmática menor que 63 $\mathrm{mg} / \mathrm{dL}$. 3) Outro tipo de hipoglicemia - evento com sintomas sugestivos de hipoglicemia, porém sem confirmação.

\section{EMPA}

1) Hipoglicemia confirmada - pela glicemia capilar $\leq 70 \mathrm{mg} / \mathrm{dL}$ e/ou necessidade de assistência com administração de carboidratos, glucagon ou outra medida de ressuscitação.

\section{SAXA}

1) Hipoglicemia reportada - eventos de hipoglicemia informados pelo paciente, porém sem confirmação. 2) Hipoglicemia confirmada - pela glicemia capilar $\leq 50$ $\mathrm{mg} / \mathrm{dL}$.

\section{SITA}

1) Hipoglicemia grave - um evento de hipoglicemia que necessitou de assistência com administração de carboidratos, glucagon ou outra medida de ressuscitação. 2) Hipoglicemia documentada - um evento típico de hipoglicemia acompanhado de uma concentração plasmática de glicose $\leq 70 \mathrm{mg} / \mathrm{dL}$. 3) Hipoglicemia assintomática - um evento de hipoglicemia com concentração plasmática de glicose $\leq 70 \mathrm{mg} / \mathrm{dL}$ sem sintomas clássicos. 4) Hipoglicemia provável - um evento com sintomas prováveis de hipoglicemia, porém sem medida da glicemia. 5) Hipoglicemia relativa - um evento onde a pessoa com diabetes qualquer sintoma típico de hipoglicemia e interpreta com indicativo de hipoglicemia. 


\section{REFERÊNCIAS}

1. Kharroubi AT, Darwish HM. Diabetes mellitus: The epidemic of the century. World J Diabetes. 2015;6(6):850-67.

2. Aguiree F, Brown A, Cho NH, Dahlquist G, Dodd S, Dunning T, et al. IDF diabetes atlas; 2013.

3. Bertoldi AD, Kanavos P, Franca GV, Carraro A, Tejada CA, Hallal PC, et al. Epidemiology, management, complications and costs associated with type 2 diabetes in Brazil: a comprehensive literature review. Global Health. 2013;9:62.

4. Iser BPM, Stopa SR, Chueiri PS, Szwarcwald CL, Malta DC, Monteiro HOdC, et al. Self-reported diabetes prevalence in Brazil: results from National Health Survey 2013. Epidemiologia e Serviços de Saúde. 2015;24(2):305-14.

5. American Diabetes A. 2. Classification and Diagnosis of Diabetes. Diabetes Care. 2017;40(Suppl 1):S11-S24.

6. Kahn SE, Zraika S, Utzschneider KM, Hull RL. The beta cell lesion in type 2 diabetes: there has to be a primary functional abnormality. Diabetologia. 2009;52(6):1003-12.

7. Inzucchi SE, Bergenstal RM, Buse JB, Diamant M, Ferrannini E, Nauck M, et al. Management of hyperglycemia in type 2 diabetes: a patient-centered approach: position statement of the American Diabetes Association (ADA) and the European Association for the Study of Diabetes (EASD). Diabetes Care. 2012;35(6):1364-79.

8. Inzucchi SE, Bergenstal RM, Buse JB, Diamant M, Ferrannini E, Nauck M, et al. Management of hyperglycemia in type 2 diabetes, 2015: a patient-centered approach: update to a position statement of the American Diabetes Association and the European Association for the Study of Diabetes. Diabetes Care. 2015;38(1):1409.

9. The effect of intensive treatment of diabetes on the development and progression of long-term complications in insulin-dependent diabetes mellitus. The 
Diabetes Control and Complications Trial Research Group. N Engl J Med. 1993;329(14):977-86.

10. Klein R, Klein BE, Moss SE. Relation of glycemic control to diabetic microvascular complications in diabetes mellitus. Ann Intern Med. 1996;124(1 Pt 2):90-6.

11. Nakagami T, Kawahara R, Hori S, Omori Y. Glycemic control and prevention of retinopathy in Japanese NIDDM patients. A 10-year follow-up study. Diabetes Care. 1997;20(4):621-2.

12. Intensive blood-glucose control with sulphonylureas or insulin compared with conventional treatment and risk of complications in patients with type 2 diabetes (UKPDS 33). UK Prospective Diabetes Study (UKPDS) Group. Lancet. 1998;352(9131):837-53.

13. Seaquist ER, Anderson J, Childs B, Cryer P, Dagogo-Jack S, Fish L, et al. Hypoglycemia and diabetes: a report of a workgroup of the American Diabetes Association and the Endocrine Society. Diabetes Care. 2013;36(5):1384-95.

14. Gordon JP, Evans M, Puelles J, McEwan PC. Factors Predictive of Weight Gain and Implications for Modeling in Type 2 Diabetes Patients Initiating Metformin and Sulfonylurea Combination Therapy. Diabetes Ther. 2015.

15. Garber AJ, Abrahamson MJ, Barzilay JI, Blonde L, Bloomgarden ZT, Bush MA, et al. Aace/Ace comprehensive diabetes management algorithm 2015. Endocr Pract. 2015;21(4):438-47.

16. Standards of medical care in diabetes--2015: Approaches to Glycemic Treatment. Diabetes Care. 2015;38 Suppl:S41.

17. Diabetes SBd. Diretrizes da Sociedade Brasileira de Diabetes: 2014-2015. In: Diabetes SBd, editor.: CIP-BRASIL. CATALOGAÇÃO NA PUBLICAÇÃO; 2015. p. 390.

18. American Diabetes A. 8. Pharmacologic Approaches to Glycemic Treatment. Diabetes Care. 2017;40(Suppl 1):S64-S74. 
19. Berkowitz SA, Krumme AA, Avorn J, et al. Initial choice of oral glucoselowering medication for diabetes mellitus: A patient-centered comparative effectiveness study. JAMA Internal Medicine. 2014;174(12):1955-62.

20. DeFronzo RA, Goodman AM. Efficacy of metformin in patients with noninsulin-dependent diabetes mellitus. The Multicenter Metformin Study Group. N Engl J Med. 1995;333(9):541-9.

21. Rossetti L, DeFronzo RA, Gherzi R, Stein P, Andraghetti G, Falzetti G, et al. Effect of metformin treatment on insulin action in diabetic rats: in vivo and in vitro correlations. Metabolism. 1990;39(4):425-35.

22. United Kingdom Prospective Diabetes Study (UKPDS). 13: Relative efficacy of randomly allocated diet, sulphonylurea, insulin, or metformin in patients with newly diagnosed non-insulin dependent diabetes followed for three years. BMJ. 1995;310(6972):83-8.

23. Kahn SE, Haffner SM, Heise MA, Herman WH, Holman RR, Jones NP, et al. Glycemic durability of rosiglitazone, metformin, or glyburide monotherapy. N Engl J Med. 2006;355(23):2427-43.

24. Brown JB, Conner C, Nichols GA. Secondary failure of metformin monotherapy in clinical practice. Diabetes Care. 2010;33(3):501-6.

25. Hauber AB, Mohamed AF, Johnson FR, Falvey H. Treatment preferences and medication adherence of people with Type 2 diabetes using oral glucose-lowering agents. Diabet Med. 2009;26(4):416-24.

26. Inzucchi SE, Bergenstal RM, Buse JB, Diamant M, Ferrannini E, Nauck M, et al. Management of hyperglycaemia in type 2 diabetes, 2015: a patient-centred approach. Update to a position statement of the American Diabetes Association and the European Association for the Study of Diabetes. Diabetologia. 2015;58(3):42942.

27. Folse HJ, Mukherjee J, Sheehan JJ, Ward AJ, Pelkey RL, Dinh TA, et al. Delays in treatment intensification with oral antidiabetic drugs and risk of microvascular and macrovascular events in patients with poor glycaemic control: An individual patient simulation study. Diabetes Obes Metab. 2017. 
28. Paul SK, Klein K, Thorsted BL, Wolden ML, Khunti K. Delay in treatment intensification increases the risks of cardiovascular events in patients with type 2 diabetes. Cardiovasc Diabetol. 2015;14:100.

29. Schloot NC, Haupt A, Schutt M, Badenhoop K, Laimer M, Nicolay C, et al. Risk of severe hypoglycemia in sulfonylurea-treated patients from diabetes centers in Germany/Austria: how big is the problem? which patients are at risk? Diabetes Metab Res Rev. 2015.

30. Group UKHS. Risk of hypoglycaemia in types 1 and 2 diabetes: effects of treatment modalities and their duration. Diabetologia. 2007;50(6):1140-7.

31. Davidson JA. Advances in therapy for type 2 diabetes: GLP-1 receptor agonists and DPP-4 inhibitors. Cleve Clin J Med. 2009;76 Suppl 5:S28-38.

32. Inkster B, Zammitt NN, Frier BM. Drug-induced hypoglycaemia in type 2 diabetes. Expert Opin Drug Saf. 2012;11(4):597-614.

33. Blickle JF. Meglitinide analogues: a review of clinical data focused on recent trials. Diabetes Metab. 2006;32(2):113-20.

34. Yki-Jarvinen H. Thiazolidinediones. N Engl J Med. 2004;351(11):1106-18.

35. Baggio LL, Drucker DJ. Biology of incretins: GLP-1 and GIP. Gastroenterology. 2007;132(6):2131-57.

36. Buse JB, Rosenstock J, Sesti G, Schmidt WE, Montanya E, Brett JH, et al. Liraglutide once a day versus exenatide twice a day for type 2 diabetes: a 26-week randomised, parallel-group, multinational, open-label trial (LEAD-6). Lancet. 2009;374(9683):39-47.

37. Nishio S, Abe M, Ito H. Anagliptin in the treatment of type 2 diabetes: safety, efficacy, and patient acceptability. Diabetes Metab Syndr Obes. 2015;8:163-71.

38. Chinese Clinical Trial Registry: Protection of xagliptin tablets to pancreatic function on new diagnosed type 2 diabetes with csii [Available from: http://www.chictr.org.cn/showprojen.aspx?proj=11830. 
39. International clinical trials registry plataform: Mass Balance of Retagliptin to healthy subjects in a single-dose Phase I study [Available from: http://apps.who.int/trialsearch/Trial2.aspx?TriallD=ChiCTR-OPC-15006905.

40. Muto C, Dai H, Teeter JG, Johnson S, Cropp AB, Chiba K, et al. The pharmacokinetics and pharmacodynamics of PF-00734200, a DPP-IV inhibitor, in healthy Japanese subjects. Int J Clin Pharmacol Ther. 2012;50(7):505-9.

41. McKeage K. Trelagliptin: First Global Approval. Drugs. 2015;75(10):1161-4.

42. Jung E, Kim J, Kim SH, Kim S, Cho MH. Gemigliptin, a novel dipeptidyl peptidase-4 inhibitor, exhibits potent anti-glycation properties in vitro and in vivo. Eur J Pharmacol. 2014;744:98-102.

43. Kim MK, Rhee EJ, Han KA, Woo AC, Lee MK, Ku BJ, et al. Efficacy and safety of teneligliptin, a dipeptidyl peptidase-4 inhibitor, combined with metformin in Korean patients with type 2 diabetes mellitus: a 16-week, randomized, double-blind, placebo-controlled phase III trial. Diabetes Obes Metab. 2015;17(3):309-12.

44. Li J, Klemm K, O'Farrell AM, Guler HP, Cherrington JM, Schwartz S, et al. Evaluation of the potential for pharmacokinetic and pharmacodynamic interactions between dutogliptin, a novel DPP4 inhibitor, and metformin, in type 2 diabetic patients. Curr Med Res Opin. 2010;26(8):2003-10.

45. Joshi BK, Ramsey B, Johnson B, Patterson DE, Powers J, Facchine KL, et al. Elucidating the pathways of degradation of denagliptin. J Pharm Sci. 2010;99(7):3030-40.

46. Deacon CF. Dipeptidyl peptidase-4 inhibitors in the treatment of type 2 diabetes: a comparative review. Diabetes Obes Metab. 2011;13(1):7-18.

47. Poole RM, Dungo RT. Ipragliflozin: first global approval. Drugs. 2014;74(5):611-7.

48. Shing TK, Ng WL, Chan JY, Lau CB. Design, syntheses, and SAR studies of carbocyclic analogues of sergliflozin as potent sodium-dependent glucose cotransporter 2 inhibitors. Angew Chem Int Ed Engl. 2013;52(32):8401-5. 
49. Mikhail N. Remogliflozin etabonate: a novel SGLT2 inhibitor for treatment of diabetes mellitus. Expert Opin Investig Drugs. 2015;24(10):1381-7.

50. Ikeda S, Takano Y, Cynshi O, Tanaka R, Christ AD, Boerlin V, et al. A novel and selective sodium-glucose cotransporter-2 inhibitor, tofogliflozin, improves glycaemic control and lowers body weight in patients with type 2 diabetes mellitus. Diabetes Obes Metab. 2015;17(10):984-93.

51. Seino Y, Inagaki N, Haneda M, Kaku K, Sasaki T, Fukatsu A, et al. Efficacy and safety of luseogliflozin added to various oral antidiabetic drugs in Japanese patients with type 2 diabetes mellitus. Journal of Diabetes Investigation. 2015;6(4):443-53.

52. International clinical trials registry plataform: A Double Blind Placebo Controlled Study to Evaluate the Effects of Bexagliflozin on Hemoglobin A1c in Patients With Type 2 Diabetes and Increased Risk of Cardiovascular Adverse Events [Available from: http://apps.who.int/trialsearch/Trial2.aspx?TriallD=NCT02558296.

53. Amin NB, Wang X, Jain SM, Lee DS, Nucci G, Rusnak JM. Dose-ranging efficacy and safety study of ertugliflozin, a sodium-glucose co-transporter 2 inhibitor, in patients with type 2 diabetes on a background of metformin. Diabetes Obes Metab. 2015;17(6):591-8.

54. Ferrannini E, Solini A. SGLT2 inhibition in diabetes mellitus: rationale and clinical prospects. Nat Rev Endocrinol. 2012;8(8):495-502.

55. Cefalu WT, Leiter LA, Yoon KH, Arias P, Niskanen L, Xie J, et al. Efficacy and safety of canagliflozin versus glimepiride in patients with type 2 diabetes inadequately controlled with metformin (CANTATA-SU): 52 week results from a randomised, double-blind, phase 3 non-inferiority trial. Lancet. 2013;382(9896):94150.

56. Nauck MA, Del Prato S, Meier JJ, Duran-Garcia S, Rohwedder K, Elze M, et al. Dapagliflozin versus glipizide as add-on therapy in patients with type 2 diabetes who have inadequate glycemic control with metformin: a randomized, 52-week, 
double-blind, active-controlled noninferiority trial. Diabetes Care. 2011;34(9):201522.

57. Roden M, Weng J, Eilbracht J, Delafont B, Kim G, Woerle HJ, et al. Empagliflozin monotherapy with sitagliptin as an active comparator in patients with type 2 diabetes: a randomised, double-blind, placebo-controlled, phase 3 trial. Lancet Diabetes Endocrinol. 2013;1(3):208-19.

58. Schernthaner G, Gross JL, Rosenstock J, Guarisco M, Fu M, Yee J, et al. Canagliflozin compared with sitagliptin for patients with type 2 diabetes who do not have adequate glycemic control with metformin plus sulfonylurea: a 52-week randomized trial. Diabetes Care. 2013;36(9):2508-15.

59. Lapuerta P, Zambrowicz B, Strumph P, Sands A. Development of sotagliflozin, a dual sodium-dependent glucose transporter 1/2 inhibitor. Diabetes and Vascular Disease Research. 2015;12(2):101-10.

60. Wu JH, Foote C, Blomster J, Toyama T, Perkovic V, Sundstrom J, et al. Effects of sodium-glucose cotransporter-2 inhibitors on cardiovascular events, death, and major safety outcomes in adults with type 2 diabetes: a systematic review and meta-analysis. Lancet Diabetes Endocrinol. 2016;4(5):411-9.

61. Zinman B, Wanner C, Lachin JM, Fitchett D, Bluhmki E, Hantel S, et al. Empagliflozin, Cardiovascular Outcomes, and Mortality in Type 2 Diabetes. N Engl J Med. 2015;373(22):2117-28.

62. Martens P, Mathieu C, Verbrugge FH. Promise of SGLT2 Inhibitors in Heart Failure: Diabetes and Beyond. Curr Treat Options Cardiovasc Med. 2017;19(3):23.

63. Neal B, Perkovic V, Mahaffey KW, de Zeeuw D, Fulcher G, Erondu N, et al. Canagliflozin and Cardiovascular and Renal Events in Type 2 Diabetes. N Engl J Med. 2017;377(7):644-57.

64. Van de Laar FA, Lucassen PL, Akkermans RP, Van de Lisdonk EH, De Grauw WJ. Alpha-glucosidase inhibitors for people with impaired glucose tolerance or impaired fasting blood glucose. Cochrane Database Syst Rev. 2006(4):CD005061. 
65. Fonseca VA, Handelsman Y, Staels B. Colesevelam lowers glucose and lipid levels in type 2 diabetes: the clinical evidence. Diabetes Obes Metab. 2010;12(5):384-92.

66. Defronzo RA. Bromocriptine: a sympatholytic, d2-dopamine agonist for the treatment of type 2 diabetes. Diabetes Care. 2011;34(4):789-94.

67. Colosia AD, Palencia R, Khan S. Prevalence of hypertension and obesity in patients with type 2 diabetes mellitus in observational studies: a systematic literature review. Diabetes Metab Syndr Obes. 2013;6:327-38.

68. Alvarez Guisasola F, Tofe Povedano S, Krishnarajah G, Lyu R, Mavros P, Yin D. Hypoglycaemic symptoms, treatment satisfaction, adherence and their associations with glycaemic goal in patients with type 2 diabetes mellitus: findings from the Real-Life Effectiveness and Care Patterns of Diabetes Management (RECAP-DM) Study. Diabetes Obes Metab. 2008;10 Suppl 1:25-32.

69. Turner RC, Cull CA, Frighi V, Holman RR. Glycemic control with diet, sulfonylurea, metformin, or insulin in patients with type 2 diabetes mellitus: progressive requirement for multiple therapies (UKPDS 49). UK Prospective Diabetes Study (UKPDS) Group. JAMA. 1999;281(21):2005-12.

70. Mamza J, Mehta R, Donnelly R, Idris I. Important differences in the durability of glycaemic response among second-line treatment options when added to metformin in type 2 diabetes: a retrospective cohort study. Ann Med. 2016;48(4):224-34.

71. Lavalle-Gonzalez FJ, Januszewicz A, Davidson J, Tong C, Qiu R, Canovatchel W, et al. Efficacy and safety of canagliflozin compared with placebo and sitagliptin in patients with type 2 diabetes on background metformin monotherapy: a randomised trial. Diabetologia. 2013;56(12):2582-92.

72. Rosenstock J, Aggarwal N, Polidori D, Zhao Y, Arbit D, Usiskin K, et al. Doseranging effects of canagliflozin, a sodium-glucose cotransporter 2 inhibitor, as addon to metformin in subjects with type 2 diabetes. Diabetes Care. 2012;35(6):1232-8.

73. Ferrannini E, Berk A, Hantel S, Pinnetti S, Hach T, Woerle HJ, et al. Longterm safety and efficacy of empagliflozin, sitagliptin, and metformin: an active- 
controlled, parallel-group, randomized, 78-week open-label extension study in patients with type 2 diabetes. Diabetes Care. 2013;36(12):4015-21.

74. Rosenstock J, Hansen L, Zee P, Li Y, Cook W, Hirshberg B, et al. Dual addon therapy in type 2 diabetes poorly controlled with metformin monotherapy: a randomized double-blind trial of saxagliptin plus dapagliflozin addition versus single addition of saxagliptin or dapagliflozin to metformin. Diabetes Care. 2015;38(3):37683.

75. Rosenstock J, Seman LJ, Jelaska A, Hantel S, Pinnetti S, Hach T, et al. Efficacy and safety of empagliflozin, a sodium glucose cotransporter 2 (SGLT2) inhibitor, as add-on to metformin in type 2 diabetes with mild hyperglycaemia. Diabetes Obes Metab. 2013;15(12):1154-60.

76. DeFronzo RA, Lewin A, Patel S, Liu D, Kaste R, Woerle HJ, et al. Combination of empagliflozin and linagliptin as second-line therapy in subjects with type 2 diabetes inadequately controlled on metformin. Diabetes Care. 2015;38(3):384-93.

77. Taieb V, Pacou M, Schroeder M, Nielsen AT, Schubert A, Neslusan C. A Network Meta-Analysis (NMA) to Assess the Longer-Term Relative Efficacy of Canagliflozin in Patients with Type 2 Diabetes Inadequately Controlled on Metformin. Value Health. 2015;18(7):A598.

78. Shyangdan DS, Uthman OA, Waugh N. SGLT-2 receptor inhibitors for treating patients with type 2 diabetes mellitus: a systematic review and network metaanalysis. BMJ Open. 2016;6(2):e009417.

79. Kawalec P, Mikrut A, Lopuch S. The safety of dipeptidyl peptidase-4 (DPP-4) inhibitors or sodium-glucose cotransporter 2 (SGLT-2) inhibitors added to metformin background therapy in patients with type 2 diabetes mellitus: a systematic review and meta-analysis. Diabetes Metab Res Rev. 2014;30(4):269-83.

80. Ferrannini E, Fonseca V, Zinman B, Matthews D, Ahren B, Byiers S, et al. Fifty-two-week efficacy and safety of vildagliptin vs. glimepiride in patients with type 2 diabetes mellitus inadequately controlled on metformin monotherapy. Diabetes Obes Metab. 2009;11(2):157-66. 
81. Goke B, Gallwitz B, Eriksson J, Hellqvist A, Gause-Nilsson I, Investigators DC. Saxagliptin is non-inferior to glipizide in patients with type 2 diabetes mellitus inadequately controlled on metformin alone: a 52-week randomised controlled trial. Int J Clin Pract. 2010;64(12):1619-31.

82. Nauck MA, Meininger G, Sheng D, Terranella L, Stein PP, Sitagliptin Study G. Efficacy and safety of the dipeptidyl peptidase-4 inhibitor, sitagliptin, compared with the sulfonylurea, glipizide, in patients with type 2 diabetes inadequately controlled on metformin alone: a randomized, double-blind, non-inferiority trial. Diabetes Obes Metab. 2007;9(2):194-205.

83. Seck T, Nauck M, Sheng D, Sunga S, Davies MJ, Stein PP, et al. Safety and efficacy of treatment with sitagliptin or glipizide in patients with type 2 diabetes inadequately controlled on metformin: a 2-year study. Int $\mathrm{J}$ Clin Pract. 2010;64(5):562-76.

84. Filozof C, Gautier JF. A comparison of efficacy and safety of vildagliptin and gliclazide in combination with metformin in patients with Type 2 diabetes inadequately controlled with metformin alone: a 52-week, randomized study. Diabet Med. 2010;27(3):318-26.

85. Forst $\mathrm{T}$, Uhlig-Laske B, Ring A, Graefe-Mody U, Friedrich C, Herbach K, et al. Linagliptin (BI 1356), a potent and selective DPP-4 inhibitor, is safe and efficacious in combination with metformin in patients with inadequately controlled Type 2 diabetes. Diabet Med. 2010;27(12):1409-19.

86. Arechavaleta $R$, Seck $T$, Chen $Y$, Krobot KJ, O'Neill EA, Duran $L$, et al. Efficacy and safety of treatment with sitagliptin or glimepiride in patients with type 2 diabetes inadequately controlled on metformin monotherapy: a randomized, doubleblind, non-inferiority trial. Diabetes Obes Metab. 2011;13(2):160-8.

87. Kim HS, Shin JA, Lee SH, Kim ES, Cho JH, Son HY, et al. A comparative study of the effects of a dipeptidyl peptidase-IV inhibitor and sulfonylurea on glucose variability in patients with type 2 diabetes with inadequate glycemic control on metformin. Diabetes Technol Ther. 2013;15(10):810-6. 
88. Del Prato S, Camisasca R, Wilson C, Fleck P. Durability of the efficacy and safety of alogliptin compared with glipizide in type 2 diabetes mellitus: a 2-year study. Diabetes Obes Metab. 2014;16(12):1239-46.

89. Gallwitz B, Rosenstock J, Rauch T, Bhattacharya S, Patel S, von Eynatten M, et al. 2-year efficacy and safety of linagliptin compared with glimepiride in patients with type 2 diabetes inadequately controlled on metformin: a randomised, doubleblind, non-inferiority trial. Lancet. 2012;380(9840):475-83.

90. Srivastava S, Saxena GN, Keshwani P, Gupta R. Comparing the efficacy and safety profile of sitagliptin versus glimepiride in patients of type 2 diabetes mellitus inadequately controlled with metformin alone. J Assoc Physicians India. 2012;60:2730.

91. Ridderstrale M, Andersen KR, Zeller C, Kim G, Woerle HJ, Broedl UC, et al. Comparison of empagliflozin and glimepiride as add-on to metformin in patients with type 2 diabetes: a 104-week randomised, active-controlled, double-blind, phase 3 trial. Lancet Diabetes Endocrinol. 2014;2(9):691-700.

92. Kashiwagi A, Kazuta K, Goto K, Yoshida S, Ueyama E, Utsuno A. Ipragliflozin in combination with metformin for the treatment of Japanese patients with type 2 diabetes: ILLUMINATE, a randomized, double-blind, placebo-controlled study. Diabetes Obes Metab. 2015;17(3):304-8.

93. Schumm-Draeger PM, Burgess L, Koranyi L, Hruba V, Hamer-Maansson JE, de Bruin TW. Twice-daily dapagliflozin co-administered with metformin in type 2 diabetes: a 16-week randomized, placebo-controlled clinical trial. Diabetes Obes Metab. 2015;17(1):42-51.

94. Haring HU, Merker L, Seewaldt-Becker E, Weimer M, Meinicke T, Broedl UC, et al. Empagliflozin as add-on to metformin in patients with type 2 diabetes: a 24week, randomized, double-blind, placebo-controlled trial. Diabetes Care. 2014;37(6):1650-9.

95. Charbonnel B, Karasik A, Liu J, Wu M, Meininger G, Sitagliptin Study G. Efficacy and safety of the dipeptidyl peptidase-4 inhibitor sitagliptin added to 
ongoing metformin therapy in patients with type 2 diabetes inadequately controlled with metformin alone. Diabetes Care. 2006;29(12):2638-43.

96. Ahren B, Gomis R, Standl E, Mills D, Schweizer A. Twelve- and 52-week efficacy of the dipeptidyl peptidase IV inhibitor LAF237 in metformin-treated patients with type 2 diabetes. Diabetes Care. 2004;27(12):2874-80.

97. Bailey CJ, Gross JL, Pieters A, Bastien A, List JF. Effect of dapagliflozin in patients with type 2 diabetes who have inadequate glycaemic control with metformin: a randomised, double-blind, placebo-controlled trial. Lancet. 2010;375(9733):2223-33.

98. Pan C, Xing X, Han P, Zheng S, Ma J, Liu J, et al. Efficacy and tolerability of vildagliptin as add-on therapy to metformin in Chinese patients with type 2 diabetes mellitus. Diabetes Obes Metab. 2012;14(8):737-44.

99. Yang W, Guan Y, Shentu Y, Li Z, Johnson-Levonas AO, Engel SS, et al. The addition of sitagliptin to ongoing metformin therapy significantly improves glycemic control in Chinese patients with type 2 diabetes. J Diabetes. 2012;4(3):227-37.

100. Kadowaki T, Tajima N, Odawara M, Nishii M, Taniguchi T, Ferreira JC. Addition of sitagliptin to ongoing metformin monotherapy improves glycemic control in Japanese patients with type 2 diabetes over 52 weeks. J Diabetes Investig. 2013;4(2):174-81.

101. Ross SA, Rafeiro E, Meinicke T, Toorawa R, Weber-Born S, Woerle HJ. Efficacy and safety of linagliptin $2.5 \mathrm{mg}$ twice daily versus $5 \mathrm{mg}$ once daily in patients with type 2 diabetes inadequately controlled on metformin: a randomised, double-blind, placebo-controlled trial. Curr Med Res Opin. 2012;28(9):1465-74.

102. Wilding JP, Ferrannini E, Fonseca VA, Wilpshaar W, Dhanjal P, Houzer A. Efficacy and safety of ipragliflozin in patients with type 2 diabetes inadequately controlled on metformin: a dose-finding study. Diabetes Obes Metab. 2013;15(5):403-9.

103. Bailey CJ, Gross JL, Hennicken D, Iqbal N, Mansfield TA, List JF. Dapagliflozin add-on to metformin in type 2 diabetes inadequately controlled with 
metformin: a randomized, double-blind, placebo-controlled 102-week trial. BMC Med. 2013;11:43.

104. Bolinder J, Ljunggren O, Johansson L, Wilding J, Langkilde AM, Sjostrom CD, et al. Dapagliflozin maintains glycaemic control while reducing weight and body fat mass over 2 years in patients with type 2 diabetes mellitus inadequately controlled on metformin. Diabetes Obes Metab. 2014;16(2):159-69.

105. Higgins JP, Green S. Cochrane handbook for systematic reviews of interventions: Wiley Online Library; 2008.

106. Cook DJ, Mulrow CD, Haynes RB. Systematic reviews: synthesis of best evidence for clinical decisions. Ann Intern Med. 1997;126(5):376-80.

107. Lu G, Ades AE. Combination of direct and indirect evidence in mixed treatment comparisons. Stat Med. 2004;23(20):3105-24.

108. Jansen JP, Fleurence R, Devine B, Itzler R, Barrett A, Hawkins N, et al. Interpreting indirect treatment comparisons and network meta-analysis for healthcare decision making: report of the ISPOR Task Force on Indirect Treatment Comparisons Good Research Practices: part 1. Value Health. 2011;14(4):417-28.

109. Moher D, Liberati A, Tetzlaff J, Altman DG, Group P. Preferred reporting items for systematic reviews and meta-analyses: the PRISMA statement. BMJ. 2009;339:b2535.

110. Del Prato S, Nauck M, Durán-Garcia S, Maffei L, Rohwedder K, Theuerkauf A, et al. Long-term glycaemic response and tolerability of dapagliflozin versus a sulphonylurea as add-on therapy to metformin in patients with type 2 diabetes: 4year data. Diabetes, Obesity and Metabolism. 2015;17(6):581-90.

111. Salsali A, Ridderstråle $M$, Andersen $K$, Toorawa R, Woerle H. Empagliflozin (EMPA) Compared with Glimepiride (GLIM) as Add-On to Metformin (MET) for 4 Years in Patients with Type 2 Diabetes (T2DM). American Diabetes Association 76th Scientific Sessions: Oral presentation; 2016.

112. Sutton AJ, Abrams KR. Bayesian methods in meta-analysis and evidence synthesis. Stat Methods Med Res. 2001;10(4):277-303. 
113. Higgins JP, Thompson SG. Quantifying heterogeneity in a meta-analysis. Stat Med. 2002;21(11):1539-58.

114. Stream 3: summary of findings tables and GRADE assessments for network meta-analysis 2013 [Available from:

http://methods.cochrane.org/sites/methods.cochrane.org.cmi/files/public/uploads/So FTs\%20and\%20GRADE\%20for\%20NMA.pdf.

115. Fenici P, Sternhufvud C, Cain V, Mukherjee J, Rohwedder K. Dapagliflozin added to metformin is effective in achieving combined improvements in $\mathrm{HbA1c}$ and weight without hypoglycaemia over 4 years. Diabetologia. 2015;58(1):S355.

116. Nauck MA, Prato S, Meier JJ, Durán-García S, Rohwedder K, Elze M, et al. Dapagliflozin versus glipizide as add-on therapy in patients with type 2 diabetes who have inadequate glycemic control with metformin: a randomized, 52-week, doubleblind, active-controlled noninferiority trial2011; 34(9):[2015-22 pp.]. Available from: http://onlinelibrary.wiley.com/o/cochrane/clcentral/articles/422/CN$\underline{00832422 / \text { frame.html }}$ http://care.diabetesjournals.org/content/diacare/34/9/2015.full.pdf.

117. Rohwedder K, Johnsson E, Parikh S. Reduced risk of hypoglycaemic events with dapagliflozin vs glipizide as add-on therapy in type 2 diabetes mellitus: 4-year data from a phase 3 study. Diabetologia. 2014;57(1):S326.

118. Langkilde A, Nauck MA, Del Prato S, Durán-Garcia S, Rohwedder K, Theuerkauf $A$, et al. Durability of dapagliflozin vs glipizide as add-on therapies in type 2 diabetes inadequately controlled on metformin: 4-year data. Diabetologia. 2013;56:S374.

119. Del Prato S, Nauck MA, Rohwedder K, Theuerkauf A, Langkilde AM, Parikh S. Long-term efficacy and safety of add-on dapagliflozin vs add-on glipizide in patients with type 2 diabetes mellitus inadequately controlled with metformin: 2-year results. Diabetologia. 2011;54:S348.

120. Rohwedder K, Parikh SJ, Johnsson E. Lower risk of hypoglycemic events with dapagliflozin than glipizide over 4 years in a phase 3 study. Diabetes. 2014;63:A563-A4. 
121. Stenlöf K, Cain V, Rohwedder K, Johnsson E. Maintenance of weight loss with dapagliflozin vs. Glipizide as add-on to metformin over 4 years. Diabetes. 2015;64:A27.

122. Rosenstock J, Gross JL, Aguilar-Salinas C, Hissa M, Berglind N, Ravichandran S, et al. Long-term 4-year safety of saxagliptin in drug-naive and metformin-treated patients with Type 2 diabetes. Diabet Med. 2013;30(12):1472-6.

123. Rosenstock J, Gross JL, Salinas CAA, Hissa M, Berglind N, Ravichandran S, et al. Long-term safety and efficacy of saxagliptin after 4-year follow-up of patients with type 2 diabetes. Diabetes. 2011;60:A298.

124. Matthews JE, Ahren B, Ye J, Carr MC, Stewart MW. Harmony 3 year 3 Results: Albiglutide vs sitagliptin and glimepiride in patients with type 2 diabetes mellitus on metformin. Diabetologia. 2014;57(1):S337.

125. Johnson S, Ahrén B, Stewart M, Cirkel D, Yang F, Perry C. HARMONY 3: 104 week efficacy of albiglutide compared to sitagliptin and glimepiride in patients with type 2 diabetes mellitus on metformin. Diabetologia. 2013;56:S8-S9.

126. Johnson S, Ahren B, Stewart M, Cirkel D, Yang F, Perry C, editors. HARMONY 3: 104 week efficacy of albiglutide compared to sitagliptin and glimepiride in patients with type 2 diabetes mellitus on metformin. Diabetologia; 2013: SPRINGER 233 SPRING ST, NEW YORK, NY 10013 USA.

127. Foroutan N, Muratov S, Levine M. Safety and efficacy of dipeptidyl peptidase4 inhibitors vs sulfonylurea in metformin-based combination therapy for type 2 diabetes mellitus: Systematic review and meta-analysis. Clin Invest Med. 2016;39(2):E48-62.

128. Zaccardi F, Webb DR, Htike ZZ, Youssef D, Khunti K, Davies MJ. Efficacy and safety of sodium-glucose co-transporter-2 inhibitors in type 2 diabetes mellitus: systematic review and network meta-analysis. Diabetes Obes Metab. 2016;18(8):783-94.

129. Millen BE, Wolongevicz DM, Nonas CA, Lichtenstein AH. 2013 American Heart Association/American College of Cardiology/the Obesity Society Guideline for the Management of Overweight and Obesity in Adults: implications and new 
opportunities for registered dietitian nutritionists. $\mathrm{J}$ Acad Nutr Diet. 2014;114(11):1730-5.

130. American Diabetes A. 4. Lifestyle Management. Diabetes Care. 2017;40(Suppl 1):S33-S43.

131. Effect of intensive blood-glucose control with metformin on complications in overweight patients with type 2 diabetes (UKPDS 34). UK Prospective Diabetes Study (UKPDS) Group. Lancet. 1998;352(9131):854-65.

132. King P, Peacock I, Donnelly R. The UK prospective diabetes study (UKPDS): clinical and therapeutic implications for type 2 diabetes. $\mathrm{Br} \mathrm{J}$ Clin Pharmacol. 1999;48(5):643-8.

133. Garber AJ, Abrahamson MJ, Barzilay JI, Blonde L, Bloomgarden ZT, Bush $M A$, et al. Consensus Statement by the American Association of Clinical Endocrinologists and American College of Endocrinology on the Comprehensive Type 2 Diabetes Management Algorithm - 2017 Executive Summary. Endocr Pract. 2017;23(2):207-38.

134. Milech A, Angelucci A, Golbert A, Carrilho A, Ramalho A, Aguiar A. Diretrizes da sociedade brasileira de diabetes (2015-2016). São Paulo. 2016.

135. Abdul-Ghani MA, Puckett C, Triplitt C, Maggs D, Adams J, Cersosimo E, et al. Initial combination therapy with metformin, pioglitazone and exenatide is more effective than sequential add-on therapy in subjects with new-onset diabetes. Results from the Efficacy and Durability of Initial Combination Therapy for Type 2 Diabetes (EDICT): a randomized trial. Diabetes Obes Metab. 2015;17(3):268-75.

136. Esposito K, Chiodini P, Bellastella G, Maiorino MI, Giugliano D. Proportion of patients at $\mathrm{HbA} 1 \mathrm{c}$ target $<7 \%$ with eight classes of antidiabetic drugs in type 2 diabetes: systematic review of 218 randomized controlled trials with 78945 patients. Diabetes Obes Metab. 2012;14(3):228-33.

137. Oderda G, Richards K, Turpin S. Sulfonylurea Agents \& Combination Products Drug Class Review. 2013.

138. Goring S, Hawkins N, Wygant G, Roudaut M, Townsend R, Wood I, et al. Dapagliflozin compared with other oral anti-diabetes treatments when added to 
metformin monotherapy: a systematic review and network meta-analysis. Diabetes Obes Metab. 2014;16(5):433-42.

139. Leiter LA, Yoon KH, Arias P, Langslet G, Xie J, Balis DA, et al. Canagliflozin provides durable glycemic improvements and body weight reduction over 104 weeks versus glimepiride in patients with type 2 diabetes on metformin: a randomized, double-blind, phase 3 study. Diabetes Care. 2015;38(3):355-64.

140. Nathan DM, Buse JB, Davidson MB, Ferrannini E, Holman RR, Sherwin R, et al. Medical management of hyperglycemia in type 2 diabetes: a consensus algorithm for the initiation and adjustment of therapy: a consensus statement of the American Diabetes Association and the European Association for the Study of Diabetes. Diabetes Care. 2009;32(1):193-203.

141. Thomsen RW, Baggesen LM, Sogaard M, Pedersen L, Norrelund H, Buhl ES, et al. Early glycaemic control in metformin users receiving their first add-on therapy: a population-based study of 4,734 people with type 2 diabetes. Diabetologia. 2015;58(10):2247-53.

142. Neal B, Perkovic V, Mahaffey KW, de Zeeuw D, Fulcher G, Erondu N, et al. Canagliflozin and Cardiovascular and Renal Events in Type 2 Diabetes. N Engl J Med. 2017.

143. Prakash A, Risser RC, Mallinckrodt $\mathrm{CH}$. The impact of analytic method on interpretation of outcomes in longitudinal clinical trials. Int $\mathrm{J}$ Clin Pract. 2008;62(8):1147-58.

144. Fitchett D, Zinman B, Wanner C, Lachin JM, Hantel S, Salsali A, et al. Heart failure outcomes with empagliflozin in patients with type 2 diabetes at high cardiovascular risk: results of the EMPA-REG OUTCOME(R) trial. Eur Heart J. 2016;37(19):1526-34. 


\section{Apêndice}

\section{A. Contato com os autores dos artigos}

Renato Zilli <rwzilli@gmail.com>

8 de maio de 2017 11:03

Para: stefano.delprato@med.unipi.it

Dear Dr Del Prato

I am a researcher at University of Sao Paulo Medical School. My colleges and I are carrying on a systematic review about second drug alternatives after metformin failure in type 2 diabetes patients. We have identified your enlightening study: Efficacy and Safety of Dapagliflozin in Combination with Metformin in Type 2 Diabetes Patients (Clinical Trials database number NCT00660907).

To run proper meta-analysis and to recognize your valuable contribution to the subject we do need some data confirmation.

So, we would appreciate if you could provide us with the following data/answers:

\begin{tabular}{|l|l|l|l|}
\hline \multicolumn{2}{|l|}{ Number of patients completing the study } \\
\hline & Year 1 & Year 2 & Year 4 \\
\hline Dapa Group & & & \\
\hline Glipizide Group & & & \\
\hline
\end{tabular}

\begin{tabular}{|l|l|l|l|}
\hline $\begin{array}{l}\text { Number of patients were withdrawn of the study for hypoglycemic } \\
\text { events }\end{array}$ & Year 1 & $\begin{array}{l}\text { Year 2 } \\
\text { (period 1-2) }\end{array}$ & Year 4 \\
\hline & & & \\
\hline Dapa Group & & & \\
\hline Glipizide Group & & & \\
\hline
\end{tabular}




\begin{tabular}{|c|c|c|c|c|c|c|c|c|}
\hline \multicolumn{9}{|c|}{$\mathrm{HbA1c}$ during the study } \\
\hline & \multicolumn{2}{|c|}{6 months } & \multicolumn{2}{|l|}{ Year 1} & \multicolumn{2}{|l|}{ Year 2} & \multicolumn{2}{|l|}{ Year 4} \\
\hline & $\begin{array}{l}\text { HbA1c } \\
+ \text { SD }\end{array}$ & $\begin{array}{l}\mathrm{n} \\
\text { patients }\end{array}$ & $\begin{array}{l}\mathrm{HbA1c} \\
+\mathrm{SD}\end{array}$ & $\begin{array}{l}\mathrm{n} \\
\text { patients }\end{array}$ & $\begin{array}{l}\mathrm{HbA1c} \\
+\mathrm{SD}\end{array}$ & $\begin{array}{l}\mathrm{n} \\
\text { patients }\end{array}$ & $\begin{array}{l}\mathrm{HbA1c} \\
+\mathrm{SD}\end{array}$ & $\begin{array}{l}\mathrm{n} \\
\text { patients }\end{array}$ \\
\hline $\begin{array}{l}\text { Dapa } \\
\text { Group }\end{array}$ & & & & & & & & \\
\hline $\begin{array}{l}\text { Glipizide } \\
\text { Group }\end{array}$ & & & & & & & & \\
\hline
\end{tabular}

\begin{tabular}{|c|c|c|c|c|c|c|c|c|}
\hline \multicolumn{9}{|c|}{ Weight during the study (without rescue) } \\
\hline & \multicolumn{2}{|c|}{6 months } & \multicolumn{2}{|l|}{ Year 1} & \multicolumn{2}{|l|}{ Year 2} & \multicolumn{2}{|l|}{ Year 4} \\
\hline & $\begin{array}{l}\text { Weight } \\
+ \text { SD }\end{array}$ & $\begin{array}{l}\mathrm{n} \\
\text { patients }\end{array}$ & $\begin{array}{l}\text { Weight } \\
+ \text { SD }\end{array}$ & $\begin{array}{l}\mathrm{n} \\
\text { patients }\end{array}$ & $\begin{array}{l}\text { Weight } \\
+ \text { SD }\end{array}$ & $\begin{array}{l}\mathrm{n} \\
\text { patients }\end{array}$ & $\begin{array}{l}\text { Weight } \\
+ \text { SD }\end{array}$ & $\begin{array}{l}\mathrm{n} \\
\text { patients }\end{array}$ \\
\hline $\begin{array}{l}\text { Dapa } \\
\text { Group }\end{array}$ & & & & & & & & \\
\hline $\begin{array}{l}\text { Glipizide } \\
\text { Group }\end{array}$ & & & & & & & & \\
\hline
\end{tabular}

\begin{tabular}{|c|c|c|c|c|c|c|c|c|}
\hline \multicolumn{9}{|c|}{ Number of patients with hypoglycemia per group/period (not patients) } \\
\hline & \multicolumn{2}{|c|}{6 months } & \multicolumn{2}{|c|}{$\begin{array}{l}\text { Year } 1(6 \mathrm{~m}- \\
1 \mathrm{y})\end{array}$} & \multicolumn{2}{|c|}{ Year $2(1 \mathrm{y}-2 \mathrm{y})$} & \multicolumn{2}{|c|}{ Year $4(2 y-4 y)$} \\
\hline & $\begin{array}{l}\mathrm{n} \\
\text { events }\end{array}$ & $\begin{array}{l}\mathrm{n} \\
\text { patients }\end{array}$ & $\begin{array}{l}n \\
\text { events }\end{array}$ & $\begin{array}{l}\mathrm{n} \\
\text { patients }\end{array}$ & $\begin{array}{l}n \\
\text { events }\end{array}$ & $\begin{array}{l}\mathrm{n} \\
\text { patients }\end{array}$ & $\begin{array}{l}n \\
\text { events }\end{array}$ & $\begin{array}{l}\mathrm{n} \\
\text { patients }\end{array}$ \\
\hline $\begin{array}{l}\text { Dapa } \\
\text { Group }\end{array}$ & & & & & & & & \\
\hline $\begin{array}{l}\text { Glipizide } \\
\text { Group }\end{array}$ & & & & & & & & \\
\hline
\end{tabular}

- $\quad$ Could you give us further details on the recruitment process?

- $\quad$ Could you inform us if diversity/disadvantage were considered?

- $\quad$ Could you give us details of the providers' training? 
- $\quad$ Could you give us further details about the dietary and lifestyle orientation at the beginning of the study?

- $\quad$ Could you inform us if sub group analysis (sex, diabetes duration or age) were considered at year $0,1,2,3$ and 4 ?

- $\quad$ Could you give us further details on the data collection to classify the hypoglycemic events in major, minor and possible?

I look forward to hearing from you.

Sincerely,

Renato

Parikh, Shamik <Shamik.Parikh@astrazeneca.com>

9 de maio de 2017 $12: 19$

Para: Renato Zilli <rwzilli@gmail.com>

Cc: Stefano Del Prato <stefano.delprato@med.unipi.it>

Dear Renato,

Dr. Del Prato forwarded your email query to me. I am one of the AZ authors in the publication of interest to you. Looking at your questions, seems to me that almost all your responses are covered in these attached two publications that are based on the same study at different time periods. Please review them.

As you can imagine, we get a lot of similar requests and we do have limited analytical resources to answer every question. So please do look for responses in the published literature first before reaching out- this way we will all save time.

Any further questions in this regard, please direct to me.

Regards,

Shamik 
Shamik Parikh, MD

Vice President, Head of Patient Safety Center of Excellence

AstraZeneca | Global Medicines Development | GRAPSQA

200 Orchard Ridge Drive, Gaithersburg, MD 20878

$\mathrm{T}: \underline{\text { (301) 398-0299 }} \mathrm{M:} \underline{(302) 3977190}$

Shamik.parikh@astrazeneca.com

Inizio messaggio inoltrato:

Da: Renato Zilli <rwzilli@gmail.com>

Oggetto: Systematic review about gliflozins

Data: 8 maggio 2017 16:03:33 CEST

A: stefano.delprato@med.unipi.it

[Texto das mensagens anteriores oculto]

Confidentiality Notice: This message is private and may contain confidential and proprietary information. If you have received this message in error, please notify us and remove it from your system and note that you must not copy, distribute or take any action in reliance on it. Any unauthorized use or disclosure of the contents of this message is not permitted and may be unlawful.

\section{2 anexos}

깅

Dapa Vs. Glipizide_52 week.pdf

$889 \mathrm{~K}$

Dapa Vs. Glipizied_104 weeks.pdf

$394 K$

Renato Zilli <rwzilli@gmail.com>

Para: "Parikh, Shamik" <Shamik.Parikh@astrazeneca.com>

Dear Dr Shamik 
I apreciate the email that you sent last month, and I would like to let you know I already read both papers, but unfortunately the data I am looking for is not there. There are some data that I would like to include in our review, could you please share them with me?

1 - the basal weight for the 2 groups dapa/glizipide in the beginning of the study

2 - in the 4-year study, graphic B, what is the amount of patients on week 26 and week 156 in groups Dapa and Glipizide

3 - in the 4-year study, what was the amount of patientes that presented an $\mathrm{HbA} 1 \mathrm{c} \leq 7$ on week 156 and week 208?

Thank you in advance.

Best regards,

\section{Renato}

2017-05-09 12:19 GMT-03:00 Parikh, Shamik <Shamik.Parikh@astrazeneca.com>:

Dr Renato Zilli <contato@drzilli.com.br> 1 de fevereiro de 2017 09:52

Para: afshin.salsali@boehringer-ingelheim.com

Dear Dr. Salsali

I am an endocrinologist in Brazil and I am interested in the publications of the EMPA-REG $\mathrm{H} 2 \mathrm{H}-\mathrm{SU}$ trial, and I am caring on a systematic review and I would like to include your fine study.

I would like to know if there is a preview date for the publication of the 208-week extension of this study presented at the last ADA meeting.

Thank you.

Best regards

Renato

Dr. Renato Zilli

Endocrinologista CRM 110.130/SP - drzilli.com.br I Facebook.com/drzilli

Rua Barata Ribeiro, 237, cj 91 Bela Vista - São Paulo/SP 01308-000 Fone (11) 3257-

4267

Hospital Moriah - Av Moaci, 974 Moema - São Paulo/SP 04083-002 Fone (11) 5080-7890 
afshin.salsali@boehringer-

ingelheim.com <afshin.salsali@boehringer-ingelheim.com>
2 de fevereiro de 2017 09:45

Para: contato@drzilli.com.br

Dear Dr Zilli,

The manuscript is under review and we plan to publish it within the next couple of month.

Best regards,

Afshin

Renato Zilli <rwzilli@gmail.com>

Para: afshin.salsali@boehringer-ingelheim.com

Dear Dr Salsali

Let me introduce myself: I'm a researcher at Sao Paulo University in Brazil. I'm conducting with a team a systematic review about treatment options after metformin failure. We select your study, registered at Clinical Trials database number NCT01167881 - Efficacy and Safety of Empagliflozin (BI 10773) With Metformin in Patients with Type 2 Diabetes.

Unfortunately, we didn't find all the information that we need in the articles you published.

At this moment, we will appreciate if you could provide some unpublished data:

There was some inconsistency of data in the publications, outcome observed.

\begin{tabular}{|l|l|l|l|}
\hline \multicolumn{2}{|l|}{ Number of patients completing the study } \\
\hline & Year 1 & Year 2 & Year 4 \\
\hline Empa Group & & & \\
\hline Glimepiride Group & & & \\
\hline
\end{tabular}

There was some inconsistency of data in the publications. 


\begin{tabular}{|l|l|l|l|}
\hline $\begin{array}{l}\text { Number of patients were withdrawn of the study for hypoglycemic } \\
\text { events }\end{array}$ & Year 1 & $\begin{array}{l}\text { Year 2 } \\
\text { (period 1-2) }\end{array}$ & $\begin{array}{l}\text { Year 4 } \\
\text { (period 2-4) }\end{array}$ \\
\hline & & & \\
\hline Empa Group & & & \\
\hline Glimepiride Group & & & \\
\hline
\end{tabular}

We didn't find the results of 6 months published, and we had difficult to determine the set analyzed.

\begin{tabular}{|c|c|c|c|c|c|c|c|c|}
\hline \multicolumn{9}{|c|}{$\mathrm{HbA1c}$ during the study } \\
\hline & \multicolumn{2}{|c|}{6 months } & \multicolumn{2}{|l|}{ Year 1} & \multicolumn{2}{|l|}{ Year 2} & \multicolumn{2}{|l|}{ Year 4} \\
\hline & $\begin{array}{l}\text { HbA1c } \\
+S D\end{array}$ & $\begin{array}{l}\mathrm{n} \\
\text { patients }\end{array}$ & $\begin{array}{l}\mathrm{HbA1c} \\
+\mathrm{SD}\end{array}$ & $\begin{array}{l}\mathrm{n} \\
\text { patients }\end{array}$ & $\begin{array}{l}\text { HbA1c } \\
+S D\end{array}$ & $\begin{array}{l}\mathrm{n} \\
\text { patients }\end{array}$ & $\begin{array}{l}\text { HbA1c } \\
+S D\end{array}$ & $\begin{array}{l}\mathrm{n} \\
\text { patients }\end{array}$ \\
\hline $\begin{array}{l}\text { Dapa } \\
\text { Group }\end{array}$ & & & & & & & & \\
\hline $\begin{array}{l}\text { Glipizide } \\
\text { Group }\end{array}$ & & & & & & & & \\
\hline
\end{tabular}

We didn't find the results of 6 months published, and we had difficult to determine the set analyzed without rescue.

\begin{tabular}{|c|c|c|c|c|c|c|c|c|}
\hline \multicolumn{9}{|c|}{ Weight during the study (without rescue) } \\
\hline & \multicolumn{2}{|c|}{6 months } & \multicolumn{2}{|l|}{ Year 1} & \multicolumn{2}{|l|}{ Year 2} & \multicolumn{2}{|l|}{ Year 4} \\
\hline & $\begin{array}{l}\text { Weight } \\
+ \text { SD }\end{array}$ & $\begin{array}{l}\mathrm{n} \\
\text { patients }\end{array}$ & $\begin{array}{l}\text { Weight } \\
+ \text { SD }\end{array}$ & $\begin{array}{l}\mathrm{n} \\
\text { patients }\end{array}$ & $\begin{array}{l}\text { Weight } \\
+ \text { SD }\end{array}$ & $\begin{array}{l}\mathrm{n} \\
\text { patients }\end{array}$ & $\begin{array}{l}\text { Weight } \\
+ \text { SD }\end{array}$ & $\begin{array}{l}\mathrm{n} \\
\text { patients }\end{array}$ \\
\hline $\begin{array}{l}\text { Empa } \\
\text { Group }\end{array}$ & & & & & & & & \\
\hline $\begin{array}{l}\text { Glimepiride } \\
\text { Group }\end{array}$ & & & & & & & & \\
\hline
\end{tabular}

The articles refer of hypoglycemia per patient and not events. 


\begin{tabular}{|c|c|c|c|c|c|c|c|c|}
\hline \multicolumn{9}{|c|}{ Number of patients with hypoglycemia per group/period (not patients) } \\
\hline & \multicolumn{2}{|c|}{6 months } & \multicolumn{2}{|c|}{$\begin{array}{l}\text { Year 1 (6m - } \\
1 \mathrm{y})\end{array}$} & \multicolumn{2}{|c|}{ Year $2(1 y-2 y)$} & \multicolumn{2}{|c|}{ Year $4(2 y-4 y)$} \\
\hline & $\begin{array}{l}\mathrm{n} \\
\text { events }\end{array}$ & $\begin{array}{l}\mathrm{n} \\
\text { patients }\end{array}$ & $\begin{array}{l}n \\
\text { events }\end{array}$ & $\begin{array}{l}\mathrm{n} \\
\text { patients }\end{array}$ & $\begin{array}{l}n \\
\text { events }\end{array}$ & $\begin{array}{l}\mathrm{n} \\
\text { patients }\end{array}$ & $\begin{array}{l}n \\
\text { events }\end{array}$ & $\begin{array}{l}\mathrm{n} \\
\text { patients }\end{array}$ \\
\hline $\begin{array}{l}\text { Empa } \\
\text { Group }\end{array}$ & & & & & & & & \\
\hline $\begin{array}{l}\text { Glimepiride } \\
\text { Group }\end{array}$ & & & & & & & & \\
\hline
\end{tabular}

- How was the recruitment of participants made at the centers? (Invitation in person, by telephone...)

- $\quad$ Did the intervention include strategies to address diversity/disadvantage?

- How was the providers' training?

- $\quad$ Can you give further details about the dietary and lifestyle orientation at the beginning of the study?

- $\quad$ How did you collect data to classified hypoglycemia events?

- Would you have a sub group analysis considering sex, diabetes duration, age (>65 years) at start $1 / 2 / 4$ years?

I am waiting for your answer.

Thank you in advance

Renato

afshin.salsali@boehringer-ingelheim.com <afshin.salsali@boehringer- 8 de maio de ingelheim.com>

Para: rwzilli@gmail.com

Dear Renato, 
Happy if we can be of any help.

Let me run your questions with my team and I will get back to you.

Best,

Afshin

Renato Zilli <rwzilli@gmail.com>

8 de maio de 2017 11:07

Para: juliorosenstock@dallasdiabetes.com

Dear Dr Rosenstock

Let me introduce myself: I'm a researcher at Sao Paulo University in Brazil. I'm conducting with a team a systematic review about treatment options after metformin failure. We select your study, registered at Clinical Trials database number NCT00121667 - Study Assessing Saxagliptin Treatment In Type 2 Diabetic Subjects Who Are Not Controlled With Metformin Alone.

Unfortunately, we didn't find all the information that we need in the articles you published.

At this moment, we will appreciate if you could provide some unpublished data of the groups placebo+metformin and Saxagliptin $5 \mathrm{mg}+$ metformin:

We didn't find the results of 6 months published, and we had difficult to determine the set analyzed.

\begin{tabular}{|c|c|c|c|c|c|c|c|c|}
\hline \multicolumn{9}{|c|}{$\mathrm{HbA} 1 \mathrm{c}$ during the study } \\
\hline & \multicolumn{2}{|c|}{6 months } & \multicolumn{2}{|c|}{ Year 1} & \multicolumn{2}{|c|}{ Year 2} & \multicolumn{2}{|c|}{ Year 4} \\
\hline & $\begin{array}{l}\mathrm{HbA1} \\
\mathrm{c}+ \\
\mathrm{SD}\end{array}$ & $\begin{array}{l}\mathrm{n} \\
\text { patient } \\
\mathrm{s}\end{array}$ & $\begin{array}{l}\mathrm{HbA1} \\
\mathrm{c}+ \\
\mathrm{SD}\end{array}$ & $\begin{array}{l}\mathrm{n} \\
\text { patient } \\
\mathrm{s}\end{array}$ & $\begin{array}{l}\mathrm{HbA1} \\
\mathrm{c}+ \\
\mathrm{SD}\end{array}$ & $\begin{array}{l}\mathrm{n} \\
\text { patient } \\
\mathrm{s}\end{array}$ & $\begin{array}{l}\mathrm{HbA1} \\
\mathrm{c}+ \\
\mathrm{SD}\end{array}$ & $\begin{array}{l}\mathrm{n} \\
\text { patient } \\
\mathrm{s}\end{array}$ \\
\hline $\begin{array}{l}\text { Placebo+metform } \\
\text { in }\end{array}$ & & & & & & & & \\
\hline $\begin{array}{l}\text { Saxagliptin } 5 \mathrm{mg} \\
+ \text { metformin }\end{array}$ & & & & & & & & \\
\hline
\end{tabular}


We didn't find the results of 6 months published, and we had difficult to determine the set analyzed without rescue.

\begin{tabular}{|c|c|c|c|c|c|c|c|c|}
\hline \multicolumn{9}{|c|}{ Weight during the study (without rescue) } \\
\hline & \multicolumn{2}{|c|}{6 months } & \multicolumn{2}{|c|}{ Year 1} & \multicolumn{2}{|c|}{ Year 2} & \multicolumn{2}{|c|}{ Year 4} \\
\hline & $\begin{array}{l}\text { Weig } \\
\mathrm{ht}+ \\
\text { SD }\end{array}$ & $\begin{array}{l}\mathrm{n} \\
\text { patient } \\
\mathrm{s}\end{array}$ & $\begin{array}{l}\text { Weig } \\
\text { ht }+ \\
\text { SD }\end{array}$ & $\begin{array}{l}\mathrm{n} \\
\text { patient } \\
\mathrm{s}\end{array}$ & $\begin{array}{l}\text { Weig } \\
\text { ht }+ \\
\text { SD }\end{array}$ & $\begin{array}{l}\mathrm{n} \\
\text { patient } \\
\mathrm{s}\end{array}$ & $\begin{array}{l}\text { Weig } \\
\text { ht }+ \\
\text { SD }\end{array}$ & $\begin{array}{l}\mathrm{n} \\
\text { patient } \\
\mathrm{s}\end{array}$ \\
\hline \multicolumn{9}{|l|}{$\begin{array}{l}\text { Placebo+metfor } \\
\min \end{array}$} \\
\hline $\begin{array}{l}\text { Saxagliptin } 5 \mathrm{mg} \\
+ \text { metformin }\end{array}$ & & & & & & & & \\
\hline
\end{tabular}

The articles refer of hypoglycemia per patient and not events.

\begin{tabular}{|l|l|l|l|l|l|l|l|l|}
\hline \multicolumn{2}{|l|}{ Number of patients with hypoglycemia per group/period (not patients) } \\
\hline & \multicolumn{2}{|l|}{$\begin{array}{l}\text { Year 1 (6m - } \\
1 \mathrm{y})\end{array}$} & $\begin{array}{l}\text { Year 2 (1y - } \\
2 \mathrm{y})\end{array}$ & \multicolumn{2}{l|}{$\begin{array}{l}\text { Year 4 (2y - } \\
4 \mathrm{y})\end{array}$} \\
\hline & $\begin{array}{l}\mathrm{n} \\
\text { event } \\
\mathrm{s}\end{array}$ & $\begin{array}{l}\mathrm{n} \\
\text { patient } \\
\mathrm{s}\end{array}$ & $\begin{array}{l}\mathrm{n} \\
\text { event } \\
\mathrm{s}\end{array}$ & $\begin{array}{l}\mathrm{n} \\
\text { patient } \\
\mathrm{s}\end{array}$ & $\begin{array}{l}\mathrm{n} \\
\text { event } \\
\mathrm{s}\end{array}$ & $\begin{array}{l}\mathrm{n} \\
\text { patient } \\
\mathrm{s}\end{array}$ & $\begin{array}{l}\mathrm{n} \\
\text { event } \\
\mathrm{s}\end{array}$ & $\begin{array}{l}\mathrm{n} \\
\text { patient } \\
\mathrm{s}\end{array}$ \\
\hline $\begin{array}{l}\text { Placebo+metform } \\
\text { in }\end{array}$ & & & & & & & & \\
\hline $\begin{array}{l}\text { Saxagliptin } 5 \mathrm{mg} \\
+ \text { metformin }\end{array}$ & & & & & & & & \\
\hline
\end{tabular}

- How was the recruitment of participants made at the centers? (Invitation in person, by telephone...)

- $\quad$ Did the intervention include strategies to address diversity/disadvantage?

- How was the providers' training?

- $\quad$ Can you give further details about the dietary and lifestyle orientation at the beginning of the study?

- $\quad$ How did you collect data to classified hypoglycemia events?

- Would you have a sub group analysis considering sex, diabetes duration, age (>65 
years) at start $1 / 2 / 4$ years?

I am waiting for your answer.

Thank you in advance

Renato 


\section{B. Relatório estatístico}

\section{Relatório}

4 de julho de 2017

Pesquisador principal: Renato Zilli

Instituição: Universidade de São Paulo

Objetivo: comparar diferentes tipos de intervenções clínicas por meio de uma metanalise em rede.

\section{Metodologia}

Uma análise de meta-análise em rede foi realizada com a finalidade de comparar diferentes intervenções (tratamentos: $I_{1}, I_{2}, \ldots, I_{n}$ ) com um comparador (tratamento:

C) fixado.

Os dados estavam dispostos em diferentes planilhas que continham diferentes tipos de comparações.

Como tamanho de efeito, treatment effect or effect size, foram utilizados MD (mean difference) para as variáveis contínuas e IOR (log ODDs Ratio) para as variáveis categóricas.

Os resultados estão exibidos em termos de IC95\% (intervalos de confiança de 95\%) para cada uma das comparações.

Note que para a realização da análise são necessários: média, desvio-padrão e tamanho da amostra de cada estudo para cada variável estudada, no entanto, nem sempre os dados estavam disponíveis em sua completude, tornando a execução muitas vezes aproximada do "real" (desvio-padrão estimado por meio de um intervalo de confiança -- valores estimados a partir de gráficos, dados faltantes).

Planilha 1

Os resultados foram divididos entre variáveis contínuas e categóricas

Variáveis contínuas

As variáveis são idade, IMC, peso e diabetes. Os estudos disponíveis são respectivamente: DAPA x EMPA x SAXA, DAPA x EMPA x SAXA, EMPA x SAXA e DAPA.

Como exemplo tome a variável idade na Tabela 1. Nas colunas se encontram o tipo deEstudo, os Tratamentos, o "MD, o erro-padrão de MD e por fim os limites do Intervalo de Confiança IC95\%.

\begin{tabular}{lllrrrr} 
Estudo & Tratamento 1 & Tratamento 2 & MD & ErroPadrao & \multicolumn{1}{c}{ Cl95I } & \multicolumn{1}{c}{ CI95u } \\
DAPA & comparador & Dapaflozina & 0,105110762 & 0,07071536 & $-0,033488796$ & 0,24371032 \\
EMPA & comparador & Emplagliflozina & $-0,048306945$ & 0,050990011 & $-0,14824553$ & 0,05163164 \\
SAXA & comparador & Saxagliptina & 0,010106358 & 0,104030439 & $-0,193789557$ & 0,214002272
\end{tabular}




\section{Tabela 1}

Note que para todos os estudos, o IC95\% contém o 0 , indicando que não há significância estatística entre a respectiva intervenção e o comparador.

Na Tabela 2, exibimos a matriz de comparações entre os tratamentos MD e na tabela 3 a matriz de IC95\% (Limite inferior: Colunas que terminam com a letra "I") e (Limite superior: Colunas que terminam com a letra "u").

\begin{tabular}{|c|c|c|c|c|}
\hline & Comparador & Dapaflozina & Emplagliflozina & Saxagliptina \\
\hline Comparador & 0 & $\quad 0,105110762$ & $-0,048306945$ & 0,010106358 \\
\hline Dapaflozina & $-0,105110762$ & 0 & $-0,153417707$ & $-0,095004404$ \\
\hline Emplagliflozina & 0,048306945 & 0,153417707 & 0 & 0,058413303 \\
\hline Saxagliptina & $-0,010106358$ & 0,095004404 & $-0,058413303$ & 0 \\
\hline
\end{tabular}

Tabela 2

\begin{tabular}{lrrrrrrrr} 
& Comparador.ci95I & \multicolumn{3}{c}{ Dapaflozina.ci95I } & Emplagliflozina.ci95l & Saxagliptina.ci95I & Comparador.ci95u & Dapaflozina.ci95u Emplagliflozina.ci95u Saxagliptina.ci95u \\
Comparador & 0 & $-0,033488796$ & $-0,14824553$ & $-0,193789557$ & 0 & 0,24371032 & 0,05163164 & 0,214002272 \\
Dapaflozina & $-0,24371032$ & 0 & $-0,324290637$ & $-0,341547263$ & 0,033488796 & 0 & 0,017455223 & 0,151538455 \\
Emplagliflozina & $-0,05163164$ & $-0,017455223$ & 0 & $-0,168657754$ & 0,14824553 & 0,324290637 & 0 & 0,285484359 \\
Saxagliptina & $-0,214002272$ & $-0,151538455$ & $-0,285484359$ & 0 & 0,193789557 & 0,341547263 & 0,168657754
\end{tabular}

Tabela 3

Na tabela 4 são exibidas as medidas de heterogeneidade: $\mathbf{Q}, \mathbf{d f}, \mathbf{p}$-valor, tau e $I^{2}$.

$\begin{array}{lcccccc}\mathrm{Q} & \mathrm{df} & & \mathrm{p} \text {-valor } & \text { tau } & 12 & \\ 5,74122 \mathrm{E}-28 & & 0 & \mathrm{NA} & & 0 & \end{array}$

Tabela 4

Como há apenas um estudo para cada tipo de intervenção as medidas acima citadas não fazem sentido.

Variáveis categóricas

As variáveis são gênero, etnia (por categoria), hipertensão e dislipidemia. Os estudos disponíveis são respectivamente: DAPA x EMPA x SAXA, DAPA x EMPA x SAXA, EMPA x DAPA e EMPA x DAPA.

Como exemplo tome a variável gender (gênero), na Tabela 5. Nas colunas encontram-se o tipo de estudo, os Tratamentos, o IOR, o erro-padrão de IOR e os limites do IC95\%.

\begin{tabular}{lllrrrrr} 
Estudo & Tratamento 1 & Tratamento 2 & IOR & Erro-padrão & \multicolumn{1}{c}{ CI95I } & \multicolumn{1}{c}{ CI95u } \\
DAPA & comparador & Dapaflozina & $-0,04033$ & 0,142129301 & $-0,318896356$ & 0,238240265 \\
EMPA & comparador & Emplagliflozina & $-0,08082$ & 0,102580758 & $-0,281873998$ & 0,120235184 \\
SAXA & comparador & Saxagliptina & 0 & 0,209413448 & $-0,410442815$ & 0,410442815
\end{tabular}

Tabela 5

Note que para todos os estudos, o IC95\% contém o 0, indicando que não há significância estatística entre a respectiva intervenção e o comparador. 
$\mathrm{Na}$ Tabela 6, exibimos a matriz de comparações entre os tratamentos MD e na Tabela 7 a matriz de IC95\% (Limite inferior: Colunas que terminam com a letra "I") e (Limite superior: Colunas que terminam com a letra "u").

\begin{tabular}{lrrrrr} 
& Comparador & Dapaflozina & Emplagliflozina & \multicolumn{2}{c}{ Saxagliptina } \\
Comparador & 0 & $-0,040328045$ & $-0,080819407$ & $1,11022 \mathrm{E}-16$ \\
Dapaflozina & 0,040328045 & 0 & $-0,040491361$ & 0,040328045 \\
Emplagliflozina & 0,080819407 & 0,040491361 & 0 & 0,080819407 \\
Saxagliptina & $-1,11022 \mathrm{E}-16$ & $-0,040328045$ & $-0,080819407$ & 0
\end{tabular}

Tabela 6

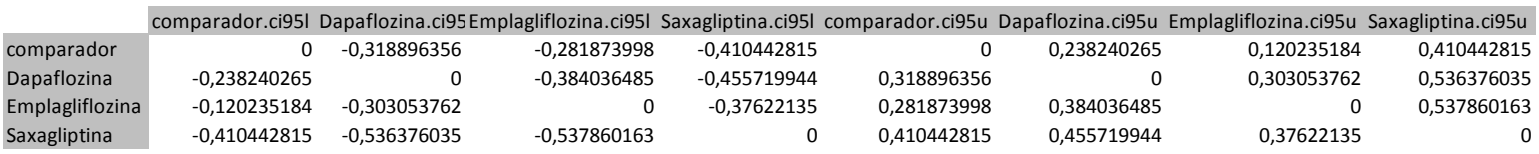

Tabela 7

Na Tabela 8 são exibidas as medidas de heterogeneidade: $\mathbf{Q}$, df, $\mathbf{p}$-valor, tau e $I^{2}$.

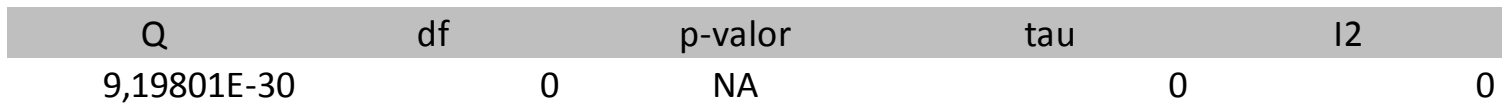

Tabela 8

Como há apenas um estudo para cada tipo de intervenção as medidas acima citadas não fazem sentido.

Planilha 2

A planilha 2 trata da evolução do $\mathrm{HbA} 1 \mathrm{c}$ no tempo. Os resultados estão exibidos no arquivo "Resultados2.xlsx" de acordo com o tempo (cada tempo em uma aba). Os resultados são semelhantes aos obtidos na planilha 1 (contínuas).

Planilha 3

$\mathrm{Na}$ planilha 3 foram feitas estimativas do tamanho de amostra em cada estudo. A estimativa foi realizada utilizando como distribuição de probabilidade uma curva normal de média $\mu$ e desvio-padrão $\sigma$ calculado pelos dados fornecidos na planilha 2 .

Os resultados estão dispostos abaixo entre as Tabelas 9 e 14 expressos em termos de IOR e IC95.

$\begin{array}{rrrr}\text { Tempo } & \text { DAPAint.p } & \text { DAPAint.n } & \text { n.estimado }<7 \\ 0 & 0,2375 & 400 & 95 \\ 0,5 & 0,398305085 & 354 & 141 \\ 1 & 0,429906542 & 321 & 138 \\ 2 & 0,35193133 & 233 & 82 \\ 3 & 0,219047619 & 105 & 23 \\ 4 & 0,265822785 & 79 & 21\end{array}$

Tabela 9 


$\begin{array}{rcrr}\text { Tempo } & \text { DAPAcom. } p & \text { DAPAcom.n } & \text { n.estimado }<7 \\ 0 & 0,206982544 & 401 & 83 \\ 0,5 & 0,550847458 & 354 & 195 \\ 1 & 0,431746032 & 315 & 136 \\ 2 & 0,264423077 & 208 & 55 \\ 3 & 0,137254902 & 102 & 14 \\ 4 & 0,197183099 & 71 & 14\end{array}$

Tabela 10

$\begin{array}{rlrr}\text { Tempo } & \text { EMPAint.p } & \text { EMPAint.n } & \text { n.estimado<7 } \\ 0 & 0,454901961 & 765 & 348 \\ 0,5 & 0,511396011 & 702 & 359 \\ 1 & 0,505426357 & 645 & 326 \\ 2 & 0,500917431 & 545 & 273 \\ 3 & 0,498789346 & 413 & 206 \\ 4 & 0,487671233 & 365 & 178\end{array}$

Tabela 11

$\begin{array}{rrrr}\text { Tempo } & \text { EMPAcom. } p & \text { EMPAcom. } n & \text { n.estimado<7 } \\ 0 & 0,143589744 & 780 & 112 \\ 0,5 & 0,630901288 & 699 & 441 \\ 1 & 0,507389163 & 609 & 309 \\ 2 & 0,439824945 & 457 & 201 \\ 3 & 0,361643836 & 365 & 132 \\ 4 & 0,181069959 & 243 & 44\end{array}$

Tabela 12

\begin{tabular}{rlccr} 
Tempo & SAXAint.p & SAXAint.n & n.estimado $<7$ \\
0 & 0,005376344 & & 186 & \\
0,5 & 0,720430108 & & 186 & \multicolumn{2}{c}{134} \\
1 & NA & NA & NA \\
2 & NA & NA & NA \\
3 & NA & NA & NA \\
4 & NA & NA & NA
\end{tabular}

Tabela 13

\begin{tabular}{|c|c|c|c|}
\hline Tempo & SAXAcom.p & SAXAcom.n & n.estimado $<7$ \\
\hline 0 & 0,005714286 & 175 & 1 \\
\hline 0,5 & 0,005714286 & 175 & 1 \\
\hline 1 & NA & NA & NA \\
\hline 2 & NA & NA & NA \\
\hline 3 & NA & NA & NA \\
\hline 4 & NA & NA & NA \\
\hline
\end{tabular}

Tabela 14

Os resultados são análogos a planilha 1 (categóricas).

Planilha 4

Na planilha 4 havia mais tipos de estudos que as demais planilhas. Para o estudo SITA havia dois tipos de intervenções (SITA1 e SITA2) e dois tipos de comparador (Glimepirida e placebo). Como nos outros estudos não havia mais de um 
comparador, foi preciso fixar um dos comparadores para que a fosse possível criar a rede. Glimepirida foi fixada por haver um maior número de observações.

Vale notar que as intervenções (SITA1 e SITA2) são comparadas.

Os resultados são exibidos na Tabela 15 e as interpretações são análogas as da planilha 1 (categórica).

\begin{tabular}{|c|c|c|c|c|c|c|}
\hline Estudo & Tratamento 1 & Tratamento 2 & IOR & Erro-padrão & Cl95I & $\mathrm{Cl} 95 \mathrm{u}$ \\
\hline DAPA & comparador & DAPA & 2,9184406 & 0,240918858 & 2,446248316 & 3,390632884 \\
\hline EMPA & comparador & EMPA & 2,492850052 & 0,223531341 & 2,054736674 & 2,93096343 \\
\hline SAXA & comparador & SAXA & 0,020783666 & 0,325595092 & $-0,617370988$ & 0,658938321 \\
\hline SITA & comparador & SITA1 & 2,574287636 & 0,253895216 & 2,076662156 & 3,071913116 \\
\hline SITA & comparador & SITA2 & 1,88904779 & 0,206499852 & 1,484315517 & 2,293780063 \\
\hline SITA & SITA1 & SITA2 & $-0,685239846$ & 0,284505284 & $-1,242859956$ & $-0,127619736$ \\
\hline
\end{tabular}

Tabela 15

Planilha 5

A planilha 5 trata da variação do peso no tempo, começando no tempo "6 meses" pois a variação é em relação ao baseline. Os resultados estão exibidos no arquivo "Resultados5.xIsx" de acordo com o tempo (cada tempo em uma aba). Os resultados são semelhantes aos obtidos na planilha 1 (contínuas).

\begin{tabular}{|c|c|c|c|c|c|}
\hline \multicolumn{6}{|c|}{6 meses } \\
\hline Estudo & Tratamento 1 Tratamento 2 & MD & ErroPadrao & $\mathrm{Cl} 95 \mathrm{I}$ & $\mathrm{Cl} 95 \mathrm{u}$ \\
\hline EMPA & comparador EMPA & 1,7446 & 0,0626017 & & 1,86729706 \\
\hline
\end{tabular}

Tabela 16

\begin{tabular}{|c|c|c|c|c|c|c|}
\hline \multicolumn{7}{|c|}{1 ano } \\
\hline Estudo & Tratamento 1 & Tratamento 2 & MD & ErroPadrao & $\mathrm{Cl95I}$ & $\mathrm{Cl} 95 \mathrm{u}$ \\
\hline DAPA & comparador & DAPA & 1,481459287 & 0,089390043 & 1,306258023 & 1,656660551 \\
\hline EMPA & comparador & EMPA & 1,682996396 & 0,065763457 & 1,554102389 & 1,811890404 \\
\hline
\end{tabular}

Tabela 17

\begin{tabular}{llllrrr} 
& & \multicolumn{2}{c}{2 anos } & \multicolumn{2}{c}{ C195I } & \multicolumn{2}{c}{ Cl95u } \\
Estudo & Tratamento 1 Tratamento 2 & MD & ErroPadrao & Cl, & \\
DAPA & comparador & DAPA & 1,461612293 & 0,106832531 & 1,252224381 & 1,671000205 \\
EMPA & comparador & EMPA & 1,372855372 & 0,069949392 & 1,235757082 & 1,509953661
\end{tabular}

Tabela 18

\begin{tabular}{|c|c|c|c|c|c|}
\hline \multicolumn{6}{|c|}{3 anos } \\
\hline Estudo & Tratamento 1 Tratamento 2 & MD & ErroPadrao & $\mathrm{Cl95I}$ & $\mathrm{Cl} 95 \mathrm{u}$ \\
\hline EMPA & comparador EMPA & 2,450997186 & 0,098065377 & 2,25879258 & 2,643201793 \\
\hline
\end{tabular}

Tabela 19 
Note que no tempo "quatro anos", na comparação (indireta) DAPA x EMPA o IC95\% não contém o zero indicando que há uma diferença significativa. Isso ocorre, pois, a medida MD para EMPA x COMPARADOR é aproximadamente 2,5 vezes a mais que MD para DAPA x COMPARADOR, Tabela 20.

\begin{tabular}{lllrrrr} 
& & \multicolumn{2}{c}{4 anos } & \multicolumn{2}{c}{ Cl954 } \\
Estudo & Tratamento 1 & Tratamento 2 & MD & ErroPadrao & \multicolumn{2}{c}{ Cl951 } \\
DAPA & comparador & DAPA & 1,07260452 & 0,123919513 & 0,829726738 & 1,315482303 \\
EMPA & comparador & EMPA & 2,627641554 & 0,113665455 & 2,404861356 & 2,850421751
\end{tabular}

Tabela 20

Uma nota sobre sensibilidade e heterogeneidade

A análise de sensibilidade é desejável uma vez que se espera que as conclusões não dependam do processo de escolha/elegibilidade dos estudos considerados. Por exemplo, se há um estudo que não contém as informações de maneira clara, podemos rodar a meta-análise em rede duas vezes, considerando e não considerando o estudo, sendo desejável que as conclusões não discordem nos dois casos. No entanto, como há somente 1 estudo por comparação (1 estudo DAPACOMPARADOR, 1 estudo EMPA-COMPARADOR), não é possível proceder uma análise de sensibilidade.

O mesmo ocorre para a análise de heterogeneidade. Como a heterogeneidade (dada através da estatística de teste qui-quadrado $Q$, juntamente com os graus de liberdade e um p-valor associado, e também estatística 12 e tau) mede a discordância entre os estudos para uma fixada comparação, não é possível realizar tal análise, pois há somente 1 estudo por comparação. 